\title{
SAR MAP OF GEL PHANTOM IN A 64MHz MRI BIRDCAGE BY FIBER-OPTIC THERMOMETRY AND FDTD SIMULATION
}

\author{
A Thesis Presented to the \\ Electrical Engineering Faculty of \\ California Polytechnic State University, San Luis Obispo \\ In Partial Fulfillment \\ of the Requirements for the Degree \\ Master of Science in Electrical Engineering
}

by

Chirag Mukesh Patel

February 2011 
(C) 2011

Chirag Mukesh Patel

ALL RIGHTS RESERVED 


\section{COMMITTEE MEMBERSHIP}

TITLE: $\quad$ SAR Map of Gel Phantom in a $64 \mathrm{MHz}$

MRI Birdcage by Fiber-Optic Thermometry and FDTD

Simulation

AUTHOR: $\quad$ Chirag M. Patel

DATE SUBMITTED: $\quad$ February 14, 2011

COMMITTEE CHAIR: Dennis Derickson

COMMITTEE MEMBER: Robert B. Szlavik

COMMITTEE MEMBER: Lily Hsu Laiho

COMMITTEE MEMBER: Gaurav Gupta

COMMITTEE MEMBER: Ross Venook 


\begin{abstract}
SAR Map of Gel Phantom in a 64MHz MRI Birdcage by Fiber-Optic Thermometry and FDTD Simulation
\end{abstract}

Chirag M. Patel

As implantable medical devices are being used more often to treat medical problems for which pharmaceuticals don't suffice, it is important to understand their interactions with commonly used medical modalities. The interactions between medical implants and Magnetic Resonance Imaging machines have proven to be a risk for patients with implants.

Implanted medical devices with elongated metallic components can create harmful levels of local heating in a Magnetic Resonance Imaging (MRI) environment [1]. The heating of a biological medium under MRI is monitored via the Specific Absorption Rate (SAR). SAR, defined as power absorbed per unit mass (W/kg), can be calculated as $S A R=\frac{\sigma|E|^{2}}{2 \rho}=c \frac{\Delta T}{t}$, where $\sigma$ is electrical conductivity of the medium in units of $\frac{S}{m},|E|$ is the magnitude of the applied electric field in units of $\frac{V}{m}$, and $\rho$ is the density of the medium in units of $\frac{\mathrm{kg}}{\mathrm{m}^{3}}$. For continuous, uniform power deposition this can be measured experimentally as a rise in temperature over time $(\Delta \mathrm{T} / \mathrm{t})$, where $c$ is the specific heat capacity of the medium in units of $\frac{J}{g \times K}$. To understand the SAR induced in-vivo, a phantom (Figure 2.10) is used to conduct in-vitro experiments, as it provides a controllable and repeatable experimental setup. 
In order to experiment in the phantom, an understanding of the background SAR distribution and in turn the exposure field distribution of the phantom is required as per the ASTMF2182-09 standard [2]. In this work, the background SAR distribution of an ASTM standard torso phantom is measured and studied via fiber optic thermometry. The measurements are compared with an electromagnetic model simulated via FDTD, demonstrating agreement between $10-25 \%$. A custom exposure and data collection setup (including oscilloscope, function generator, RF amplifier, directional coupler, and Neoptix Omniflex Fiber Optic Thermometry system) was integrated and automated using NI LabView.

The purpose of this thesis is to map the field distribution in a torso phantom under RF exposure from a $64 \mathrm{MHz}$ MRI RF Birdcage, compare the results to an electromagnetic simulation, and finally conclude the accuracy of this method for field measurements in a standard torso phantom. Understanding the capabilities and accuracy of the fiber optic thermometry method will ultimately allow researchers to successfully apply this method to monitor background fields in their respective experimental setups (related to MRI implant heating) and understand its limitations. 


\section{ACKNOWLEDGEMENTS}

I would like to first thank my mentors and co-workers at Boston Scientific, Gaurav Gupta, Ross Venook, Matt McDonald, and Rafael Carbunaru for their support and guidance with this thesis. Along with this, I would like to thank them for their patience and the time they took to not only to bring me up to speed on the MRI project but also their enthusiasm in helping me to succeed. I would also like to thank Sriram Kharidehal for providing the basis for electromagnetic simulations presented in this thesis. Additionally, I would like to thank Dr. Derickson for having the patience and interest to help me pursue a project in cooperation with industry. His advice and assistance on this thesis have played a critical role in its successful completion. In addition to Dr. Derickson, I would like to thank the other members of my thesis committee Dr. Laiho and Dr. Szlavik for their support.

Beyond co-workers and Cal Poly Faculty, I would like to thank the friends I have made during my time at Cal Poly who have continued to support my endeavors even after college. In addition, I would like to thank my aunt Sushila, my uncle Suryakant and my cousins Sagar and Shivani who helped me transition to California from New York and have supported me throughout my time here. I would also like to thank all of my aunts, uncles, and grandparents who have played a critical role in my upbringing, and have made many sacrifices for my success. Additionally I would like to thank my sister for being there for me no matter how much we squabbled.

Finally, but most importantly I would like to thank my parents. The sacrifices

they have made for me are endless. The values they have instilled in me are the reason for any success I have achieved. 


\section{TABLE OF CONTENTS}

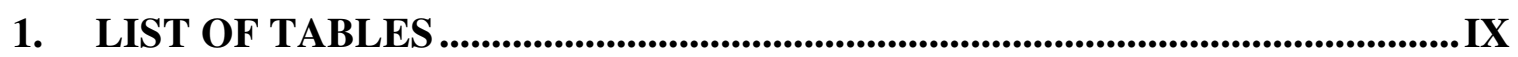

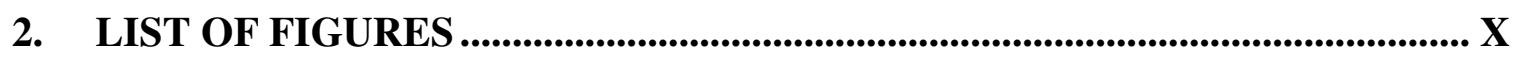

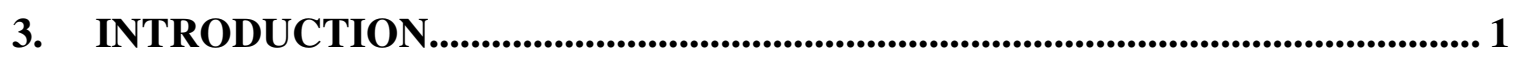

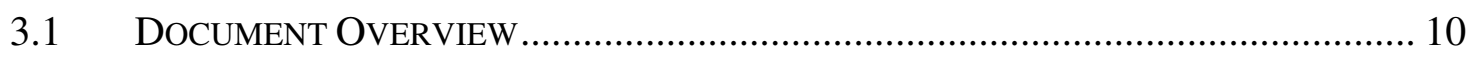

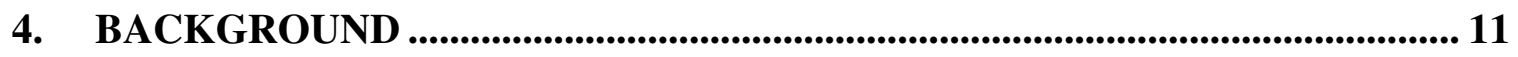

4.1 MAGNETIC ReSONANCE IMAGING (RF COIL) ................................................ 11

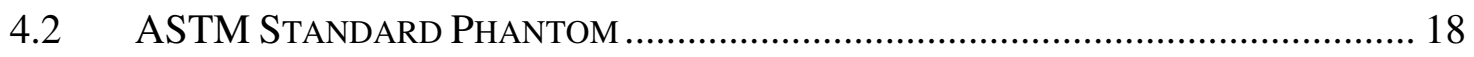

4.3 FinIte DifFERENCE TIME Domain Method (SEMCAD) ……........................ 21

4.4 EXPERIMENTAL SAR MAPPING TECHNIQUES ….............................................. 24



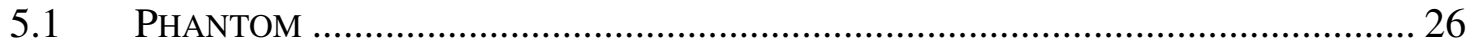



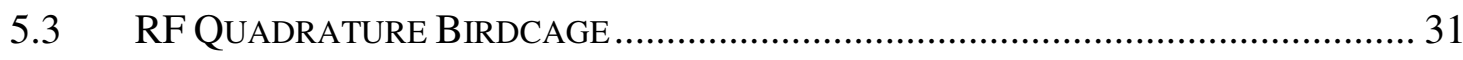

5.4 MEASUREMENTS ...................................................................................... 35

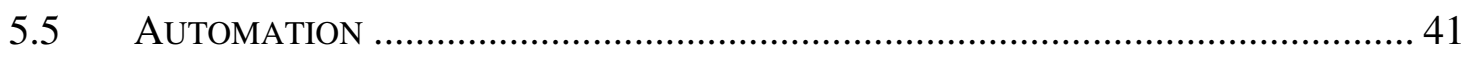

6. FDTD SIMULATIONS ............................................................................................... 45

7. RESULTS AND ANALYSIS ....................................................................................... 48

8. CONCLUSIONS AND FUTURE WORK ............................................................... 60




10. APPENDIX - RAW TEMPERATURE DATA........................................ 67 


\section{LIST OF TABLES}

TAble 1: NeOPtix Fiber Optic Temperature SEnSORS SPECIFICATION ........................ 30

TABle 2: Power InPUt to BiRdCAge For RF heAting MEASUREMENTS........................ 35 


\section{LIST OF FIGURES}

Figure 1.1: Boston Scientific Precision Spinal Cord Stimulator [3], Medtronic AdaPta PaCemaker [4], St. Jude Medical Fortify ICD [5] (LeFT to Right): MILLIONS OF PATIENTS ARE IMPLANTED EVERY YEAR AND THE NUMBER CONTINUES TO GROW

Figure 1.2: NuMBER OF MRI PROCEDURES OVER A DECADE: MRI SCANS ARE HERE TO STAY AND WILL CONTINUE TO BE A PROMINENT PART OF THE HEALTHCARE SYSTEM



FIGURE 1.3: OFTEN, PATIENTS WITH IMPLANTED MEDICAL DEVICES GET AN MRI SCAN EVEN THOUGH IT IS CONTRAINDICATED FOR MOST DEVICES. A DBS PATIENT, SUSTAINED A LESION (HEMORRHAGE) IN THE LEFT PORTION OF HIS BRAIN RESULTING IN SEVERE DYSARTHIRA AND HEMIPARESIS AFTER GETTING AN MRI SCAN WITH A CONDITIONALLY SAFE MEDTRONIC DBS SYSTEM UNDER MRI SETTINGS WHICH DID NOT ADHERE TO THE DEVICE SPECIFICATIONS. A CT SCAN REVEALS THE LESION SURROUNDING THE LEFT DBS ELECTRODE [7] .................................................. 5

FIGURE 1.4: SCHEMATIC DIAGRAM OF TANGENTIAL (TO THE LEAD) E-FIELD COUPLING TO LEAD, CAUSING HIGH CURRENTS ALONG THE LEAD AND IN TURN CONTACT HEATING AT THE ENDS. 6

FIGURE 1.5: FDTD SIMULATED CROSS SECTION OF RF E-FIELDS GENERATED IN HUMAN DURING MRI SCAN. E-FIELD DISTRIBUTIONS GENERATED IN HUMANS DURING AN MRI ARE VERY INTRICATE AND DIFFICULT TO REPLICATE [11] ................................. 8

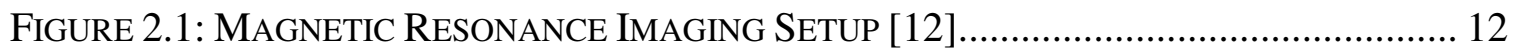


FIGURE 2.2: MRI MACHINES ARE COMPOSED OF A STATIC MAGNET, GRADIENT COILS, AND AN RF COIL. THE UNIFORM STATIC MAGNET FIELD GENERATED BY THE SUPERCONDUCTING MAGNET IS IN THE Z DIRECTION $\left(\mathrm{B}_{0}\right)$. THE GRADIENT FIELDS ARE VARIED ALONG THE X, Y, AND Z DIRECTIONS $\left(\mathrm{B}_{\mathrm{Z}}\right)$, AND THE CIRCULARLY POLARIZED

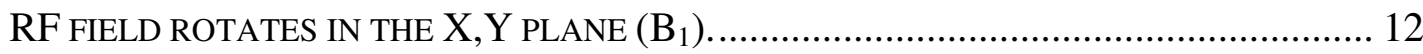

FIGURE 2.3: WHEN PLACED IN A STATIC MAGNETIC FIELD, HYDROGEN PROTONS ORIENT THEMSELVES IN ONE OF TWO STATES: PARALLEL OR ANTI-PARALLEL TO THE $\mathrm{B}_{0}$ FIELD(ANTI-PARALLEL BEING THE STATE WITH HIGH ENERGY). 14

FIGURE 2.4: THE HYDROGEN PROTONS PRECESS ABOUT THE AXIS OF THE STATIC MAGNETIC FIELD AT THE LARMOR FREQUENCY 14

FIGURE 2.5: WHEN PROTONS ABSORB RF ENERGY AT THE LARMOR FREQUENCY, THEY BEGIN TO PRECESS IN A HIGHER ENERGY STATE 15

FIGURE 2.6: RF BIRDCAGE COIL IS USED TO GENERATE A CIRCULARLY POLARIZED HOMOGENOUS B1 FIELD [13] 15

FIGURE 2.7: WHEN THE RF POWER IS TURNED OFF, THE PROTONS RETURN TO THE LOWER ENERGY STATE AND EMIT RF ENERGY, FROM WHICH THE TISSUE TYPE CAN BE DETERMINED BASED ON THE TIME IT TOOK TO RETURN TO THE LOWER ENERGY STATE, ALSO KNOWN AS THE RELAXATION TIME. 16

FIGURE 2.8: AN RF PULSE IS APPLIED TO EXCITE NUCLEI TO A HIGHER ENERGY STATE, AND WHEN THE RF IS TURNED OFF, THE NUCLEI RELAX BACK TO THE LOWER ENERGY STATE, RELEASING ENERGY. THIS RELEASED ENERGY DECAYS IN A FORM KNOWN AS FREE INDUCTION DECAY WHICH IS A FUNCTION OF LOCAL TISSUE PROPERTIES AND THIS ALLOWS FOR CONTRAST BETWEEN TISSUES IN MRI. 16 
FIGURE 2.9: MAGNETIC FIELD (A/M) DISTRIBUTION OF A LOADED (RECTANGULAR PHANTOM) QUADRATURE RF BIRDCAGE COIL (64MHZ) AT Z=0 ........................... 17

Figure 2.10: ASTM StANDARd PHANTOM TO MOdEL HuMAn TORSO [2] ...................... 19

FiguRE 2.11: Simulated SAR DistRIBUTION AT DEPTHS (INTO PAGE) Y=6.9CM, 9.65CM, AND 11.9CM FROM PHANTOM LID FROM LEFT TO RIGHT ...................................... 20

Figure 2.12: 3D YeE CELL WITH E AND H FIELD COMPONENTS [15] ............................. 23

FIGURE 3.1: ASTM TORSO PHANTOM DIMENSIONS ................................................... 27

FIGURE 3.2: 10CM TITANIUM ROD FOR NORMALIZATION AND REFERENCE ....................... 27



Figure 3.4: Ti-Rod IN PHANTOM WITH FIBER OPTIC TEMPERATURE PROBE ..................... 29

FIGURE 3.5: EXPERIMENTAL SETUP - SingLE SPLINE MEASUREMENT 14 PROBES ............. 30

Figure 3.6: Custom Neoptix Fiber Optic Probe .................................................... 30

FIGURE 3.7: 64MHz RF QUADRATURE BIRDCAGE (LOOKING INTO BORE) USED FOR MEASUREMENTS (LEFT), 20KW RF AMPLIFIER (RIGHT) ....................................... 31

FIGURE 3.8: THE FUNCTION GENERATOR SOURCES THE RF AMPLIFIER WITH A GATING SIGNAL AND THE RF SIGNAL. THE AMPLIFIER GATES THE RF SIGNAL, AND AMPLIFIES IT

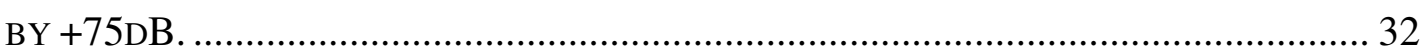

FIGURE 3.9: THE AMPLIFIED RF SIGNAL IS PASSED THROUGH A HYBRID SPLITTER WHICH SPLITS THE SIGNAL AND ADDS A 90 DEGREE PHASE OFFSET BETWEEN THE TWO SIGNALS

FIGURE 3.10: THE TWO ORTHOGONAL SIGNALS FROM THE HYBRID SPLITTER ARE THEN SENT TO TWO RUNGS OF THE BIRDCAGE (WHICH HAS A TOTAL OF 16 RUNGS), AND THE BIRDCAGE GENERATES A CIRCULARLY POLARIZED MAGNETIC FIELD $\left(\mathrm{B}_{1}\right)$. 33 
Figure 3.11: AUtOMATEd MRI RF HEATING MEASUREMENT SETUP 34

FIGURE 3.12: MEASUREMENT GRID (210 TOTAL MEASUREMENT POINTS 5X3X15) .... 36

FIGURE 3.13: SimUlATED SPLINE ALONG X-AXIS REQUIRING MOST POINTS TO CHARACTERIZE ( 4TH ORDER POLYNOMIAL). 37

FIGURE 3.14: SimULATED SPLINE ALONG Y-AXIS REQUIRING MOST POINTS TO CHARACTERIZE (2ND ORDER POLYNOMIAL) 38

FIGURE 3.15: SiMULATED SPLINE ALONG Z-AXIS REQUIRING MOST POINTS TO CHARACTERIZE (4TH ORDER POLYNOMIAL) 38

Figure 3.16: TEMPERATURE VS. TIME FOR A SPline 1 (14 POINTS ALONG Z-AXIS) Starting from P2 $2_{\mathrm{M} 1}$ (Probe 2 Module 1 ) to P $3_{\mathrm{M} 4}$ (Probe 3 Module 4). P1 $1_{\mathrm{M} 1}$ IS THE ATTACHED TO THE TITANIUM ROD, AND P4 $4_{M 4}$ IS AMBIENT TEMPERATURE, BOTH OF WHICH ARE IRRELEVANT FOR THIS STUDY. 40

Figure 3.17: MRI RF HeAting ExPERIMENT Automation Front PANEL (LABView).. 42 FIGURE 3.18: FIVE REPEAT MEASUREMENTS WERE TAKEN OF SPLINE 3 (14 POINT MEASUREMENTS). THE STANDARD DEVIATION IN THE MEASUREMENTS REMAINED BELOW 36\% AND IMPROVED TO BELOW 15\% FOR HIGH SAR REGIONS TOWARDS THE CENTER OF THE SPLINE 43

Figure 4.1: 16 Rung RF QuAdRATURe BirdCAGE CoIl Simulation Model 46

FIGURE 4.2: SEMCAD FDTD SiMULATION SETUP WITH IDEAL RF QUADRATURE



FIGURE 5.1: SPATIAL DISTRIBUTION OF INDUCED SAR (NOT NORMALIZED) WITHIN THE PHANTOM LIQUID IN GE 64 MHZ QUADRATURE BIRDCAGE COIL. LOCAL TEMPERATURE WAS MEASURED WITH FIBER OPTIC TEMPERATURE PROBES. SAR IS IN 
UNITS OF W/KG, NOTE DIFFERENT COLOR SCALING BETWEEN SUBFIGURES. Y=6.9CM (A), 9.65CM (B), AND 11.9CM (C) FROM PHANTOM LID 49

FIGURE 5.2: NORMALIZED (BY DIRECTIONAL COUPLER VOLTAGE^2) SPATIAL DISTRIBUTION OF INDUCED SAR WITHIN THE PHANTOM LIQUID IN GE 64 MHZ QUADRATURE BIRDCAGE COIL. LOCAL TEMPERATURE WAS MEASURED WITH FIBER OPTIC TEMPERATURE PROBES. NORMALIZED UNITS OF W/KG/V². NOTE DIFFERENT COLOR SCALING BETWEEN SUBFIGURES. Y=6.9CM (A), 9.65CM (B), AND 11.9CM (C) FROM PHANTOM LID. 49

FIGURE 5.3: INTERPOLATED, NORMALIZED (BY DIRECTIONAL COUPLER VOLTAGE^2) SPATIAL DISTRIBUTION OF INDUCED SAR WITHIN THE PHANTOM LIQUID IN GE 64 MHZ QUADRATURE BIRDCAGE COIL. LOCAL TEMPERATURE WAS MEASURED WITH FIBER OPTIC TEMPERATURE PROBES. UNITS OF W/KG/V². Y=6.9CM (A), 9.65CM (B), AND 11.9CM (C) FROM PHANTOM LID. 50

Figure 5.4: Simulated SPATIAL SAR DistRIBUTION (W/KG) WITH TI-ROD. Y=6.9CM (A), 9.65CM (B), AND 11.9CM (C) FROM PHANTOM LID 50

Figure 5.5: MeAsured (PINK) VS. Simulated (BluE) SAR SPlines 1-15. H = 9.7117, $\mathrm{ERROR}=87 \%$ 53

Figure 5.6: MEASURED (PINK) VS. Simulated (BLUE) SAR SPlines 1-14. H = 2.55, ERROR $=24.05 \%$ 54

FIGURE 5.7: SPLINE 1 (HIGH SAR REGION) SAR MEAS. (PINK) VS. SiMULATED (BLUE) H = 2.36 55

Figure 5.8: ERRor DiSTRIBUTION BETWEEN SiMUlated AND MEASURED SAR. Y=6.9CM (A), 9.65CM (B), AND 11.9CM (C) FROM PHANTOM LID. ERROR IS GREATEST AT 
CENTER OF PHANTOM, WHERE IMPLANT RF HEATING MEASUREMENTS ARE GENERALLY NOT MADE 56

FIGURE 5.9: SPline 15 (NEAREST TI-ROD) SAR MEASURED (PINK) AND SAR SiMULATED $($ BLUE $) \mathrm{H}=2.9214$, ERROR $=71.7 \%$. DUE TO LARGE GRADIENTS, SLIGHT MISPOSITIONING OF PROBES CAN EASILY RESULT IN HIGH ERROR. 56

FIGURE 5.10: SIMULATED SAR IN TI-ROD REGION OF PHANTOM. SHARP GRADIENTS IN SAR CAN CAUSE HIGH ERROR IN MEASUREMENT AS A RESULT OF SLIGHT INACCURACIES IN PROBE PLACEMENT. 57

FIGURE 5.11: TO UNDERSTAND ERROR OF MEASURED SAR VS. SIMULATED SAR, AS A FUNCTION OF DIFFERENT RF APPLICATION TIMES, SAR WAS CALCULATED WITH DIFFERENT TIME RANGES STARTING AT 30S RANGING THROUGH THE 1800S WINDOW.58

FIGURE 5.12: ERROR VS. DELTA T (STARTING FROM 30S). INITIAL STEEP FALL OFF AND THEN DIMINISHING RETURNS INDICATE THAT IT MAY NOT BE NECESSARY TO APPLY RF POWER MUCH PAST 1100s. 58

FIGURE 5.13: LMS ERROR (MEASURED SAR) VS. SAR SIMULATED ................................ 59

FiguRE 6.1: MEASURED (LEFT) AND SIMULATED (RIGHT) SAR DiSTRIBUTION (IN

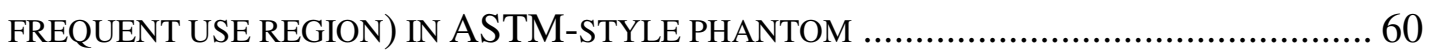

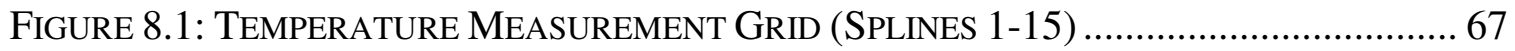
Figure 8.2: SPline 1 RAw TeMPERATure Data P2M1 - P3M4 (14 Probes PER SPline)

Figure 8.3: SPline 2 RaW TEMPERATURe Data P2M1 - P3M4 (14 ProbeS PER Spline) 69 
Figure 8.4: SPline 3 RAW TeMPERATURe DAta P2M1 - P3M4 (14 Probes PER SPline)

Figure 8.5: SPline 4 RAw TeMPERATURe Data P2M1 - P3M4 (14 Probes PER SPline)

Figure 8.6: SPline 5 RAw TeMPERATURe DAta P2M1 - P3M4 (14 Probes PER SPline)

Figure 8.7: SPline 6 RAw TeMPERATURe DAta P2M1 - P3M4 (14 Probes PER SPline)

Figure 8.8: SPline 7 RaW TEMPERATURE DATA P2M1 - P3M4 (14 ProbeS PER SPLine)

Figure 8.9: SPline 8 RAw TeMPERATure Data P2M1 - P3M4 (14 Probes PER SPline)

Figure 8.10: Spline 9 Raw Temperature Data P2M1 - P3M4 (14 Probes Per Spline)

Figure 8.11: Spline 10 RAW Temperature Data P2M1 - P3M4 (14 Probes PER

SPLINE)

Figure 8.12: SPline 11 RAW TeMPerature DATA P2M1 - P3M4 (14 Probes PER

SPliNE) 78

Figure 8.13: SPLine 12 RAW TEMPERATURE DATA P2M1 - P3M4 (14 PROBES PER

SPLINE) 79

Figure 8.14: Spline 13 RAW Temperature Data P2M1 - P3M4 (14 Probes PeR

SPLINE) 80 
Figure 8.15: SPline 14 RAW TeMPERATURE DATA P2M1 - P3M4 (14 PROBES PER

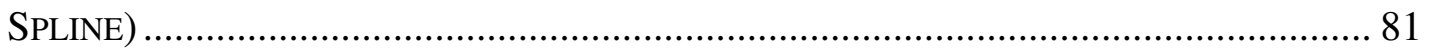

Figure 8.16: SPline 15 RAW TeMPerature DAta P2M1 - P3M4 (14 Probes PER

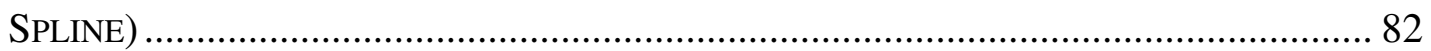




\section{INTRODUCTION}

This introduction begins by providing the reader a broad overview of implantable medical devices and their increased use in modern healthcare. This is followed by an explanation of the importance of Magnetic Resonance Imaging (MRI) machines and their prevalence as the gold standard for many medical imaging applications. Thereafter, the two medical modalities (MRI and medical implants) are bridged, via an explanation of how implanted patients are affected by the inability to safely get an MRI procedure. Finally, I define a problem statement and its relevance to the ongoing efforts to allow for safe MRI of patients with medical implants.

Implantable medical devices have revolutionized the healthcare industry. With the increasing number of patients with chronic conditions for which pharmaceuticals or other therapies are ineffective, implantable devices are becoming ever-present as long-term solutions. As corporations aim to meet clinical needs, by devoting more research and money to this fast growing field, more indications ranging from Parkinson's disease to chronic pain have become increasingly controllable via cost-effective active and passive implants. These passive and active implants often allow otherwise severely affected patients to lead their lives with normalcy. 
Vast arrays of implantable medical devices exist in the market today, many of which have been around for quite some time. Common active implants include but are not limited to, Spinal Cord Stimulators (SCS), Pacemakers, and Implantable Cardioverter Defibrillators (ICD), and even more common are passive implants such as metal screws and plates, artificial hips, and stents. This work focuses on problems that are most relevant to active devices with extended/long components, principally including pacemakers and neurostimulators. Many of these implants include a canned portion where the electronics are housed, and a "lead" which extends an electrical signal out to the relevant area of treatment (i.e. epidural space in spinal cord for SCS, right atrium for heart arrhythmia). The work presented herein does not apply solely to, but was completed with the Boston Scientific Precision Spinal Cord Stimulator system in mind [3].
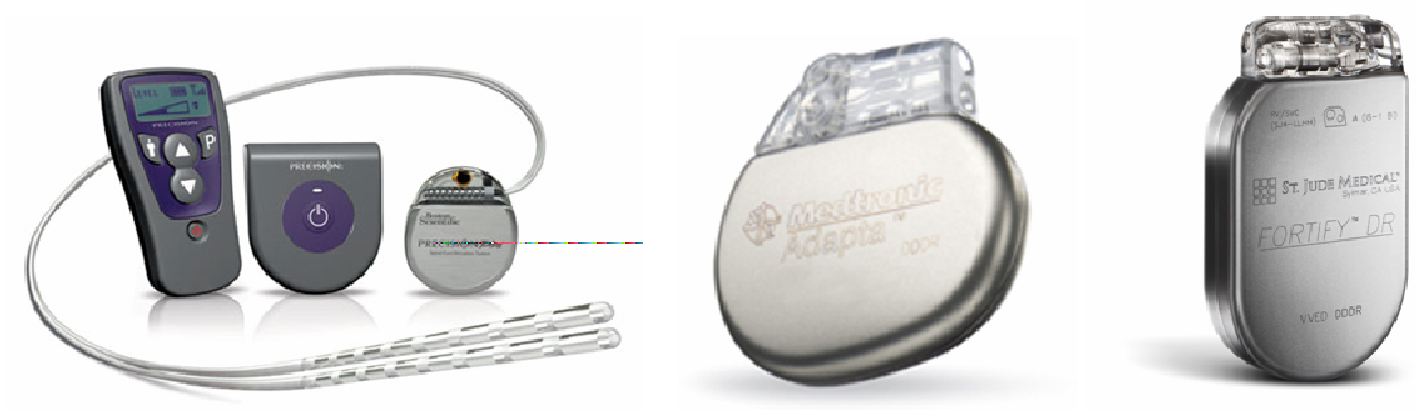

Figure 3.1: Boston Scientific Precision Spinal Cord Stimulator [3], Medtronic Adapta Pacemaker [4], St. Jude Medical Fortify ICD [5] (Left to Right): Millions of patients are implanted every year and the number continues to grow

Millions of patients are implanted every year, and the number of patients continues to increase, giving way to a market in the billions of dollars worldwide. As patients with devices are living longer, and as devices are implanted in younger patients, the same patients will often also need medical imaging. 
Magnetic Resonance Imaging is often the gold standard of imaging because of its capability to image different tissues, while also not exposing the patient to ionizing radiation unlike other imaging methods (XRay and CT Scan). Its use and advances in the technology (1.5 Tesla (T), 3.5 Tesla, Functional MRI, etc.) continue to grow at staggering rates. As illustrated in Figure 1.2, the number of MRI related procedures went from 7.7 Million in 1993 to 24.2 Million in 2003 (314\% increase). This only goes to show that MRI scans are here to stay and will continue to be a prominent part of healthcare for years to come.



Figure 3.2: Number of MRI Procedures over a Decade: MRI Scans are here to stay and will continue to be a prominent part of the healthcare system for years to come [6].

As was illustrated previously, both implantable devices (Pacemakers, ICD's, SCS's) and MRI procedures are growing at tremendous rates. This presents a problem, as the two have a variety of interactions that present safety issues. Today MRI scanners strictly contraindicate examination of patients with pacemaker or neurostimulator 
implants, and the number of patients who are denied MRI scans continues to escalate. Potential hazards include unintended stimulation, RF heating of the implant, and movement of the implant within the body. The following facts obtained from a Medtronic study are indicative of the impact of the latter situation [6]:

- MRI scans are unsafe for over 2 million Americans because they have an implanted cardiac device

- In 2004, 200,000 implantable cardiac device patients were denied an MRI scan because of safety concerns

- Every 3 minutes in the US, a patient is denied an MRI scan due to a pacemaker or defibrillator implant

- $40 \%$ of Europeans and 50-75\% of Americans with implantable devices will be recommended for an MRI scan over the lifetime of their device Due to medical need, or misunderstanding of risks or procedures, many implanted patients get MRI scans even though they are contraindicated. This results in potentially dangerous scenarios for the patient, due to interactions between the MRI and implanted devices in the patient. A case report often referenced, is that of a 56 year old patient with a history of Parkinson's disease. The patient was implanted with a Deep Brain Stimulator (DBS) system from Medtronic (that has MR Conditional safety labeling), which is used to electrically stimulate the sub thalamic nucleus region of the brain, counteracting the effects of Parkinson's disease. Seven months after his implant, the patient underwent an MRI scan of his lumbar spine, to diagnose back pain and leg pain unrelated to the original implant. Multiple scans were performed under a 1.0T system outside of the MR Conditional safety labeling guidelines, after which he was reported to have sustained a 
hemorrhage surrounding the left DBS electrode implanted in the brain. The patient was later found to have severe dysarthria (speech disorder) and right hemiparesis. The brain lesion which he sustained (Figure 1.3) was found to be a result of an interaction between the RF portion of the MRI scanner and the implanted lead, which can result in harmful levels of local heating [7]. This unfortunate incident, illustrates the potential for harm to patients with the ever increasing presence of MRI and active implantable devices.

Developing MRI safe medical implants is high priority for the medical industry, and understanding MRI machines (environment), medical implants (product), and the interactions between the two are key components to solving the issue at hand.



Figure 3.3: Often, patients with implanted medical devices get an MRI scan even though it is contraindicated for most devices. A DBS patient, sustained a lesion (hemorrhage) in the left portion of his brain resulting in severe dysarthira and hemiparesis after getting an MRI scan with a conditionally safe Medtronic DBS system under MRI settings which did not adhere to the device specifications. A CT scan reveals the lesion surrounding the left DBS electrode [7]. 
Many active implants (Figure 1.1) employ a "lead" which is essentially an insulated conductor (or several conductors), which routes the electrical signal(s) to the desired region of stimulation (i.e. epidural space for SCS) from the signal generator implant which is located in a more suitable region for an object of its size. Although each field produced by an MRI machine (RF, Gradient, and Static Magnetic) interacts with implanted metallic devices, the RF-field is considered to be the culprit for the most significant heating of lead implants [1].

The RF Coil (Figure 2.1) creates a circularly polarized $B_{1}$ field; the eddy currents induced in the body as a result of this field, concentrate to the metallic implant where higher power deposition and heating results. The RF fields generated in the human body have short wavelengths in the order of length of the lead implant $(\sim 52 \mathrm{~cm})$. Dielectric resonances and standing waves are generated as a result, and constructive interference can occur resulting in significant heating at the lead contacts. The tangential component of the E-fields along the body of the lead can be the main cause of magnified heating at the ends of the lead (electrodes, contacts) as shown in Figure 1.4. The E-Fields generated in such scenarios are often in the $80-100 \mathrm{~V} / \mathrm{m}$ range.

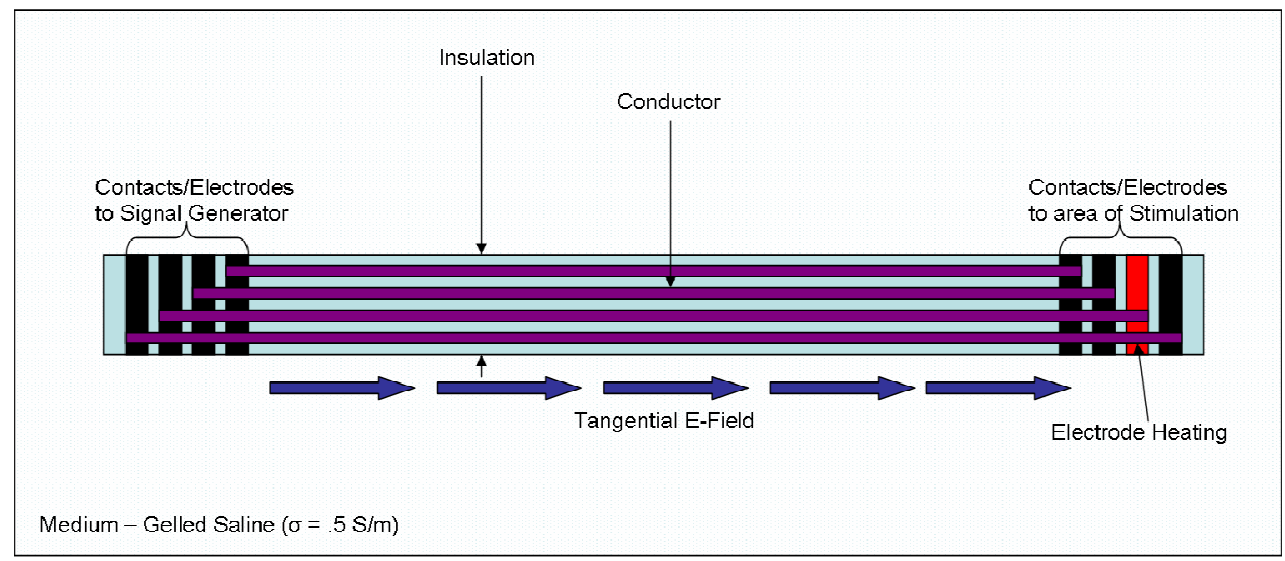

Figure 3.4: Schematic diagram of tangential (to the lead) E-field coupling to lead, causing high currents along the lead and in turn contact heating at the ends. 
Enough heating of the implant can lead to necrosis and eventually the death of tissue [8]. To understand the levels of heating that can be generated, and to design safe implants it is important to understand the E-fields to which the lead is being exposed, and hence characterization of fields produced in any given MRI experimental setup is necessary.

MRI systems are very complex, proprietary, and involve a difficult electromagnetic environment to characterize and make measurements in. When making RF heating measurements of metallic implants, the measured temperature scales with the applied fields, which can vary between MRI scan setups [9]; hence, confidence in metallic implant heating measurements is directly related to the ability to measure the background fields in the experimental setup, which is the thrust of this work. Furthermore, evidence from a study of spatial distribution of RF-induced E-Fields, conducted by Nordbeck, et al. indicates that,

local E-field strength in the direction of the implant is a critical factor in $R F$ related tissue heating. The actual E-field distribution, which is dependent on phantom/body properties and the MR-system employed, must be considered when assessing the effects of RF power deposition in implant safety investigations [10]. As illustrated in Figure 1.4, local E-field strength in the direction of the implant is the field strength tangential to the implant (implantable lead in this case). Fields generated in the human body during an MRI scan (Figure 1.5) are very intricate and difficult to replicate for experimental setups, hence it is important to understand field distributions generated in experimental setups to compare to in-vivo (in human) situations. 


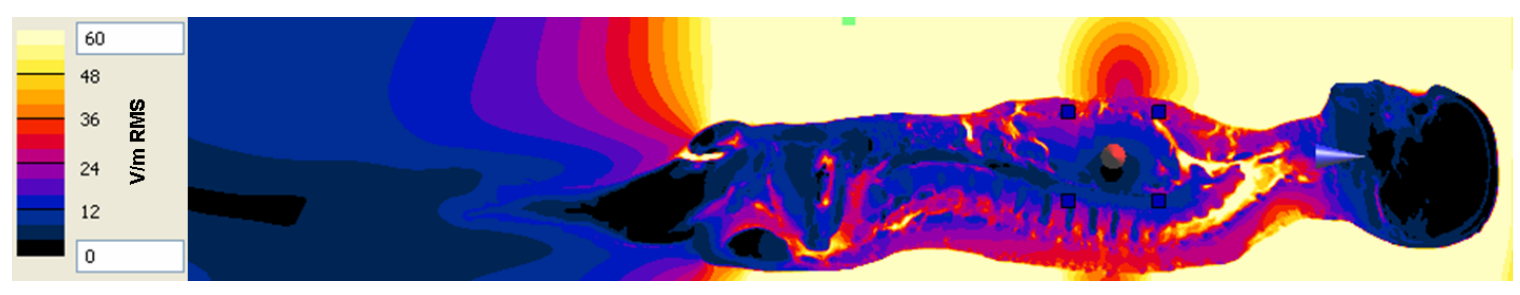

Figure 3.5: FDTD Simulated Cross section of RF E-Fields generated in human during MRI scan. EField distributions generated in humans during an MRI are very intricate and difficult to replicate [11].

Standard measurement methods and techniques (i.e. thermocouples) cannot be used in MRI, as they would not work because coupling of fields to the measurement equipment can result in false readings; hence a non-electrical method of measurement is required. In this thesis, I implement, improve, and characterize a non-interfering method for measuring MRI background fields in a biological medium similar in characteristics to human tissue (muscle). The background E-fields are proportional to a measure known as the Specific Absorption Rate (SAR), which is calculated as shown in Equation 1.1, where $\mathrm{c}$ is the specific heat capacity of the medium, $\Delta \mathrm{T} / \mathrm{t}$ is the change in temperature over a specified period of time. The SAR indicates the amount of power being absorbed by a biological sample in units of power per unit mass. The E-Field can also be calculated via this method as shown in Equation 1.2, where $\rho$ is the density and $\sigma$ is the conductivity, of the medium.

$$
\begin{aligned}
S A R & =\frac{\sigma|E|^{2}}{\rho}=c \frac{\Delta T}{t} \\
E & =\sqrt{\frac{\rho \times S A R}{\sigma}}
\end{aligned}
$$


The background SAR distribution of an experimental medium used as a standard in RF heating measurements (ASTM Standard Torso Phantom, Figure 2.10) is measured and studied via fiber-optic thermometry. Measurements are made in the medium, with it centered in a $64 \mathrm{MHz}$ quadrature whole-body birdcage coil (Figure 2.2). The measurements are compared with an electromagnetic model simulated via the Finite Different Time Domain Method in commercially available software, SEMCAD X (version 14.2). A custom exposure and data collection setup (including oscilloscope, function generator, RF amplifier, directional coupler, and Neoptix Omniflex Fiber Optic Thermometry system) was integrated and automated using NI LabView. These methods can be applied to a broad range of MRI systems. This thesis work will allow others to make use of the method with confidence, to have a full understanding of the background fields generated in respective media when in an MRI system. 


\subsection{Document Overview}

This thesis begins by providing the reader with essential background information for the topic at hand. This is followed by an explanation of the methodology used to perform the characterization of the SAR distribution generated in a given medium during an MRI scan. The measured results are then compared to simulations, and finally conclusions are drawn about the applicability and accuracy of the method used in the study. Chapter 2 provides a background discussion of MRI machines, phantoms, and the Finite Difference Time Domain method (FDTD). Chapter 3 explains in greater detail the methods used for experiments conducted herein, and provides important details of the MRI laboratory setup. Chapter 4 describes the FDTD simulation done in commercially available software SEMCAD X, model parameters, and the results. Chapter 5 summarizes the results of measurements and simulations and compares the two. Chapter 6 concludes the document with a discussion of the accuracy of the method and future work to be done. 


\section{BACKGROUND}

To follow this thesis, it is important to have a general knowledge of MRI, phantoms, and the finite difference time domain method. The following few sections aim to give the reader a brief background of MRI, an ASTM standard gel phantom, and the FDTD method used for electromagnetic simulations. The reader should aim to grasp the basic principles of each, to understand the work presented herein. For additional information, please read the materials cited in this work.

\subsection{Magnetic Resonance Imaging (RF Coil)}

This section provides the reader with a brief overview of Magnetic Resonance Imaging (MRI) principles. The most basic principles behind MRI are discussed, along with brief explanations of how each component of an MRI closed bore system functions to generate desired imaging. The RF Birdcage coil is the most relevant portion of an MRI machine to this thesis, as it is used to generate the fields which are measured herein.

MRI machines are composed of a superconducting solenoid (static magnet), gradient coils, and the RF coil (also known as the RF birdcage because of its birdcage like structure). The overall setup for a MRI machine is shown in Figure 2.1. A more 
detailed diagram of each of these structures and the corresponding fields, for a

cylindrical, 'closed-bore' system are shown in Figure 2.2.



Figure 4.1: Magnetic Resonance Imaging Setup [12]
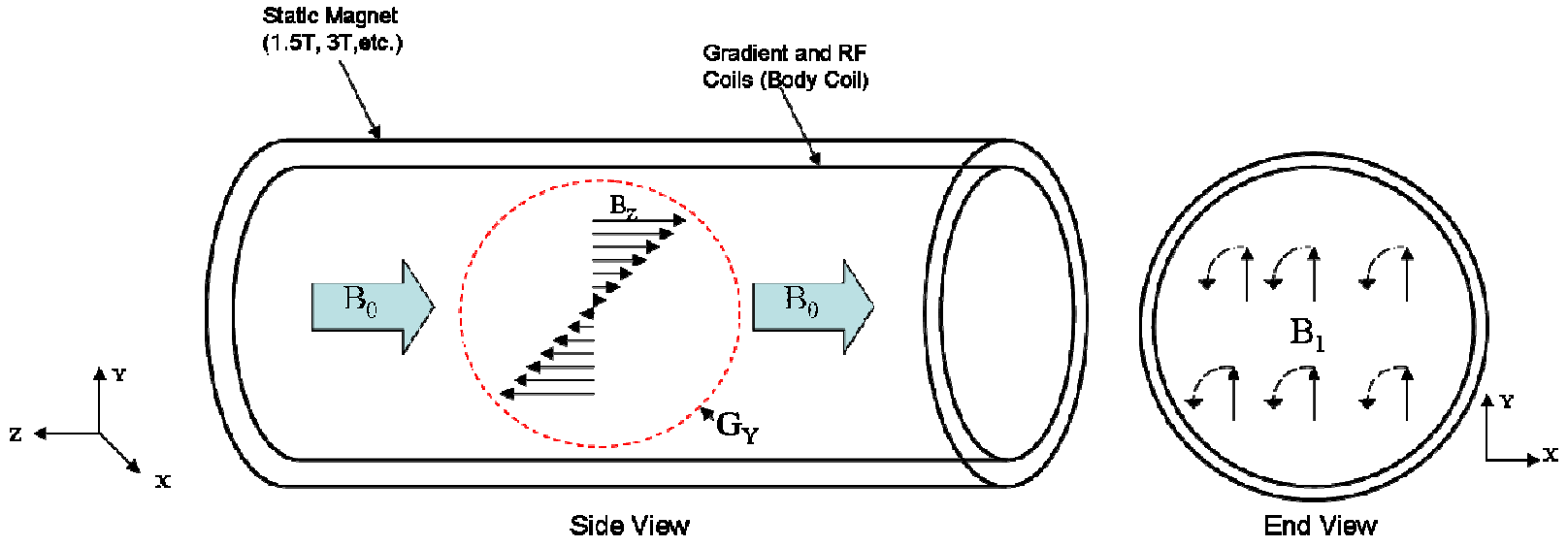

Figure 4.2: MRI machines are composed of a static magnet, gradient coils, and an RF coil. The uniform static magnet field generated by the superconducting magnet is in the $Z$ direction $\left(B_{0}\right)$. The Gradient Fields are varied along the $\mathrm{X}, \mathrm{Y}$, and $\mathrm{Z}$ directions $\left(\mathrm{B}_{\mathrm{Z}}\right)$, and the circularly polarized $\mathrm{RF}$ field rotates in the $\mathrm{X}, \mathrm{Y}$ plane $\left(\mathrm{B}_{1}\right)$. 
The uniform static magnetic field is generated along the Z-axis by a superconducting solenoid. The static magnetic field can vary between systems, though most closed-bore systems currently used in radiology centers and hospitals are $1.5 \mathrm{~T}$ or 3T. The large arrows parallel to the Z-axis, shown in Figure 2.2 represent the magnetic field generated by the solenoid; this field is referred to as the $\mathrm{B}_{0}$ field and serves to align specific nuclear species in the biological medium being imaged.

The gradient coils vary the local magnetic field strength in space, so as to obtain spatial encoding of information for the MR image. The gradient field in the $\mathrm{Y}$ direction is represented by the $\mathrm{G}_{\mathrm{Y}}$ field arrows in Figure 2.2. By sourcing the gradient coils with specific encoding sequences, selected regions and tissues in the body are imaged.

When in a static magnetic field, certain nuclear species (such as hydrogen protons that are in large concentration in the human body; others include C13, Phosphorus, Sodium) will assume one of two states - parallel or anti-parallel to the applied static field $\left(\mathrm{B}_{0}\right)$, parallel being the state with lower energy and anti-parallel, the state with higher energy (Figure 2.3). When a proton goes from one state to the other, a photon is either released or absorbed, at a frequency proportional to the strength of the static magnetic field, also known as the Larmor Frequency (64MHz for a 1.5T, $128 \mathrm{MHz}$ for a 3T), as calculated by Equation 2.1. The RF Coil generates an electromagnetic field known as the $\mathrm{B}_{1}$ field, which serves to excite the nuclei from a lower energy state and when the excitation is turned off, the RF coil listens for an electromagnetic response as the nuclei relax back to the lower energy state.

$$
f=\frac{\gamma B_{0}}{2 \pi}, \gamma \text { is the gyromagnetic ratio of Hydrogen in } \mathrm{rad} / \mathrm{s} \times T
$$




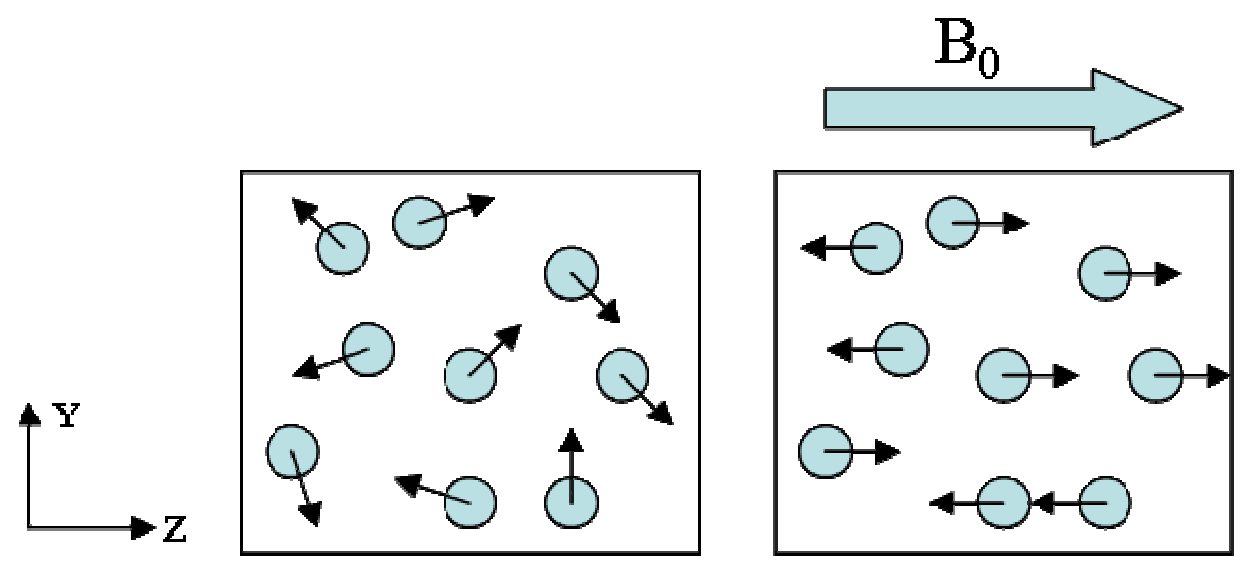

Figure 4.3: When placed in a static magnetic field, hydrogen protons orient themselves in one of two states: parallel or anti-parallel to the $B_{0}$ field(anti-parallel being the state with high energy).

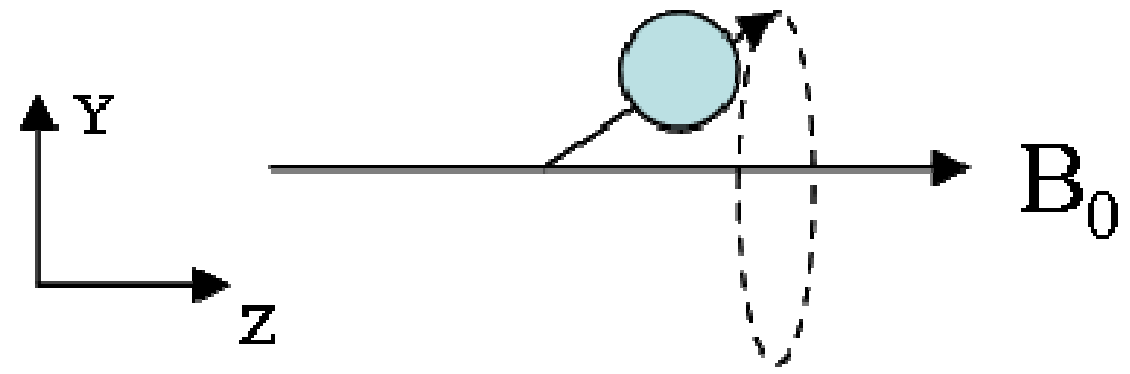

Figure 4.4: The hydrogen protons precess about the axis of the static magnetic field at the Larmor frequency

In a single MRI measurement, an RF excitation signal is applied for some period of time ( $\sim \mathrm{ms})$. When RF energy is applied at the Larmor frequency, the protons absorb the energy and begin precessing at the Larmor frequency in a state of higher energy, as shown in Figure 2.5. The nuclei are sensitive to circularly polarized fields only in a specific orientation with respect to the static magnetic field, and this circular polarization of the $\mathrm{B}_{1}$ field shown in Figure 2.2 improves the efficiency of the excitation of Hydrogen nuclei, as well as reception of RF energy, versus using a linearly polarized RF coil (in which half of the deposited energy is not productive). The RF coil (also known as a quadrature RF birdcage) used in this study is preferred because of its ability to create a 
circularly polarized homogenous $\mathrm{B}_{1}$ field. The RF birdcage, appropriately named for its structure is shown in Figure 2.6. It consists of 16 rungs which are driven with an amplified RF signal, each of which is phase offset from its neighboring rungs.

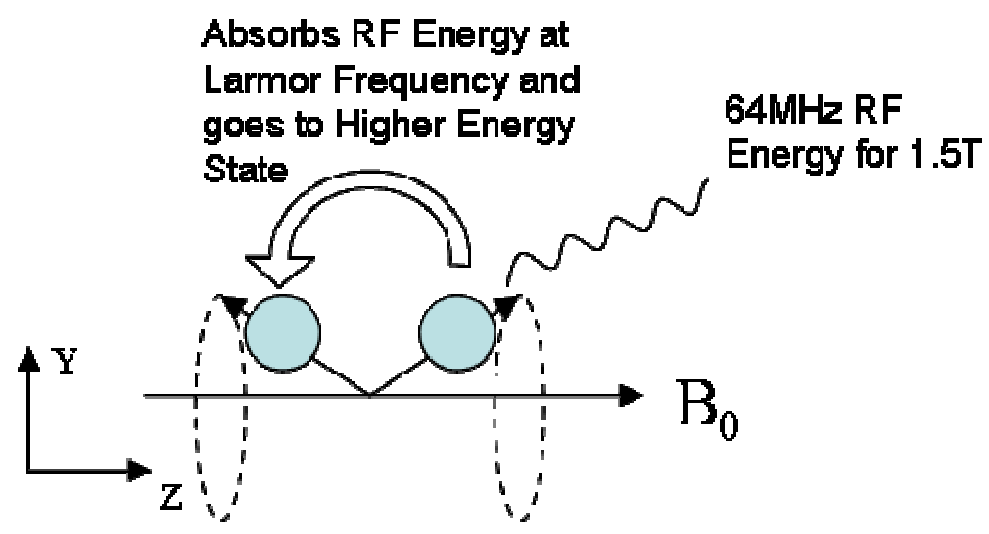

Figure 4.5: When protons absorb RF energy at the Larmor frequency, they begin to precess in a higher energy state

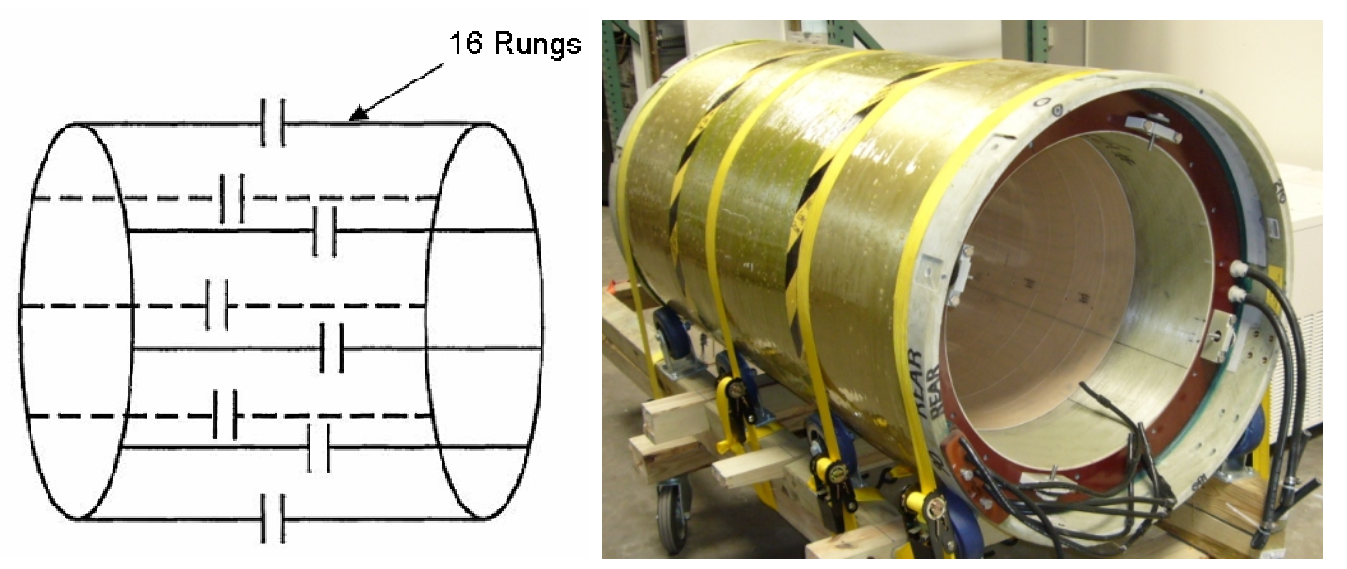

Figure 4.6: RF Birdcage Coil is used to generate a circularly polarized homogenous B1 field [13] When the RF energy is turned off, the protons relax to their original state, releasing RF energy (Figure 2.7), which is detected by the RF receive coil and analyzed for different tissue types based on the time it took for them to relax back to the lower energy state. The decay of the received signal is known as free induction decay (FID), and the basic sequence of a single scan is shown in Figure 2.8. The characteristic decay time depends 
on local tissue properties for the Hydrogen at a given place in the body, and these time constants are the basis for contrast between tissues in MRI.



Figure 4.7: When the RF power is turned off, the protons return to the lower energy state and emit RF energy, from which the tissue type can be determined based on the time it took to return to the lower energy state, also known as the relaxation time.



Figure 4.8: An RF Pulse is applied to excite nuclei to a higher energy state, and when the RF is turned off, the nuclei relax back to the lower energy state, releasing energy. This released energy decays in a form known as free induction decay which is a function of local tissue properties and this allows for contrast between tissues in MRI.

For high quality imaging, an RF coil must be able to generate a homogenous $\mathrm{B}_{1}$ field at the Larmor frequency for uniform excitation of Hydrogen nuclei, and also must 
have a high SNR for receiving signals [13]. As shown in Figure 2.9, a quadrature birdcage coil (circular polarization) can generate a homogenous $\mathrm{B}_{1}$ field while also increasing the SNR by a factor of $\sqrt{2}$ (as compared to linearly polarized coils) [13], by being able to receive the energy from the nuclei in the direction of their precession.



Figure 4.9: Magnetic field (A/m) distribution of a loaded (rectangular phantom) quadrature RF birdcage coil $(64 \mathrm{MHz})$ at $\mathrm{Z}=0$

The transmitting RF coil will couple the most energy with the highest efficiency to the precessing nuclei, at the Larmor frequency. The high frequency results in a shorter wavelength (64MHz -> $\sim 50 \mathrm{~cm}$ in humans) which becomes increasingly comparable to the size of biological objects (humans, phantom) and as a result dielectric resonances and standing waves are generated at tissue biological boundaries [13]. More concentrated power is deposited in the patient or phantom as a result of this phenomenon and safety concerns arise for those patients with metallic implants. A measure known as the Specific Absorption Rate is used to monitor and regulate the power absorption in a patient (for safety purposes) during an MRI scan, and is calculated as shown in Equation 1.1. 
Experimentally, since the behavior of the system can change due to a variety of factors (conductivity and permittivity of biological medium, position of biological medium in the bore, loading of the birdcage, etc.), it is important to verify that the birdcage is generating the expected fields, and to have an accurate map of the resulting background SAR distribution in the test environment. Understanding the SAR distribution would allow us to pick and choose under what field exposures to study respective implantable devices, and how those fields may couple to and affect the device.

\subsection{ASTM Standard Phantom}

This section provides the reader with an overview of an ASTM standard phantom [2] and its relation to the experimental setup used herein.

Since human studies (in-vivo) are both extremely challenging and otherwise not allowed by regulatory agencies, it is important to be able to simulate human body conditions to which implantable objects will be exposed when making MRI RF heating measurements. An apparatus, know as a phantom has become industry standard for the latter purpose. It is essentially a rectangular box (often with a head) made of an electrical insulator (acrylic). The phantom is filled with fluid to a depth which has conductivity between .4 and $.8 \mathrm{~S} / \mathrm{m}$, and a dielectric constant of 60 to 100 [2]. The fluid is typically a gel or other highly viscous fluid in order to avoid heat convection, allowing for accurate localized heating measurements. The ASTMF2182-09 standard describes an ideal phantom:

Ideally a phantom should model the size and shape of the entire body and contain materials that simulate the electrical properties of the different tissues of the 
body. The electrical properties of human tissue result in a complicated current flow pattern that may not be adequately reproduced in a phantom containing just one type of conducting material. A reduced size phantom may not exhibit whole body spatial resonances that might occur in a patient. These issues may be particularly relevant for static field strength in excess of 1.5T. However, a simplified phantom is less costly and easier to construct, and in many cases will provide adequate prediction of the potential for $R F$ heating near the implant [2].

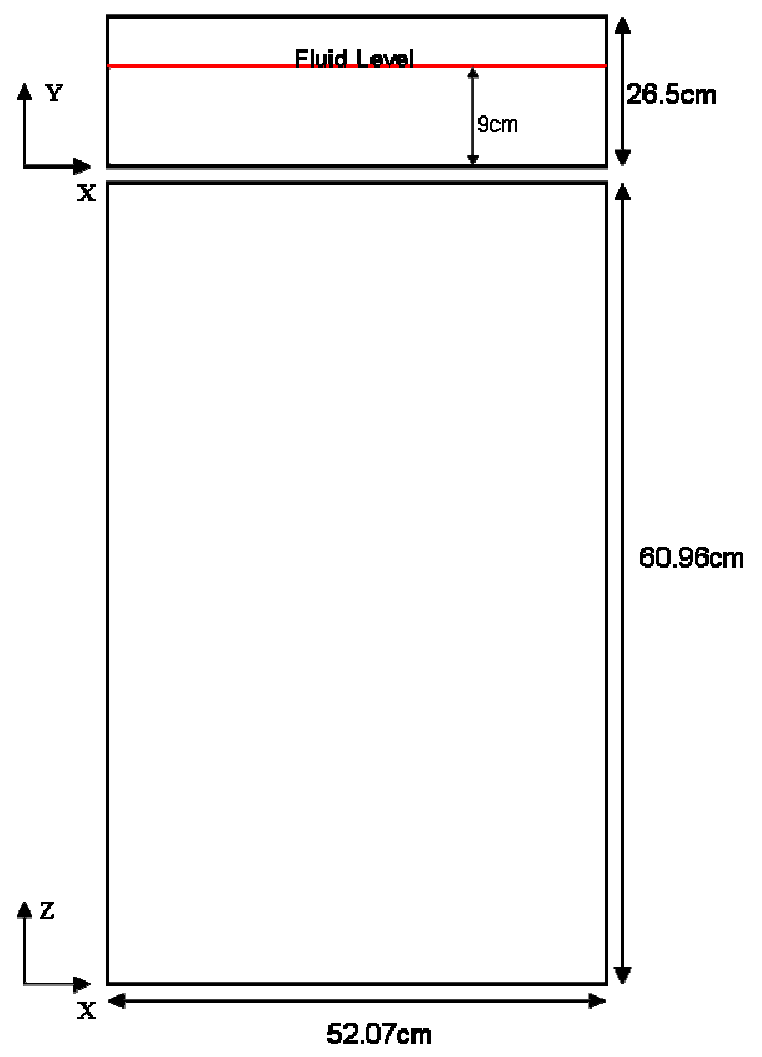

Figure 4.10: ASTM Standard Phantom to Model Human Torso [2]

In order to get an understanding of in-vivo (in-patient) behavior of an implanted device, including both applied fields and approximate surrounding electromagnetic material properties, a phantom is used to conduct in-vitro (in test setup) experiments. RF heating measurements of implants in the phantom can be used as a relative tool to 
measure heating differences between objects, but cannot be utilized as an equivalent to the human body.

As stated earlier, it is important to understand the fields generated by the RF coil (Figure 2.6) in the test environment, which in this case is the ASTM phantom. Electromagnetic simulations indicate that the SAR generated in the phantom should be symmetric and exhibit high regions at the edges and lowest regions at the center. The simulated SAR distribution in the rectangular phantom (related to E-field by Equation 1.1) is shown in Figure 2.11.
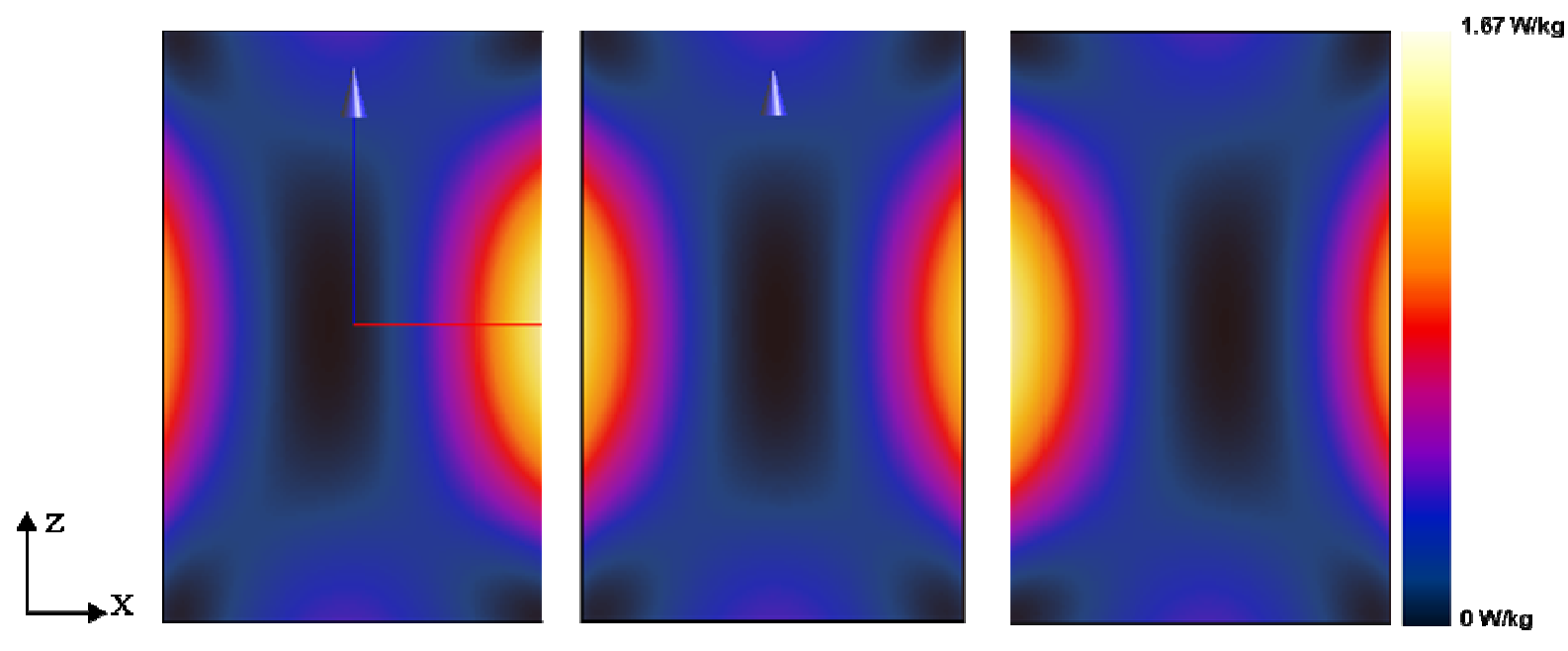

Figure 4.11: Simulated SAR distribution at depths (into page) $Y=6.9 \mathrm{~cm}, 9.65 \mathrm{~cm}$, and $11.9 \mathrm{~cm}$ from phantom lid from left to right 


\subsection{Finite Difference Time Domain Method (SEMCAD)}

This section aims to give the reader a very brief overview of methods used herein to generate simulation results for comparison to experimental results. The Finite Difference Time Domain Method (FDTD) is described, the reasons for using it, and its application to this study is explained.

Being that researchers are limited by their inability to experiment in humans, electromagnetic simulation is another method often used to predict heating performance of metallic objects in patients during MRI scans. Numerical methods often used for the three dimensional implementations of the latter, include the Finite Difference Time Domain method (FDTD), and the Method of Moments (MoM) [13]. While they are both applicable to this study, the FDTD method was chosen herein. The FDTD method is very versatile in its ability to allow broadband solutions in a single simulation (using a Gaussian pulse), it can handle varying materials over the entire space, and allows the user to visualize the flow of fields over time [14]. While it has many advantages, the FDTD method requires the entire simulation domain to be gridded appropriately down to the finest structure and shortest wavelength to get accurate results. This is especially difficult for very fine structures, as computation times can be extremely long and memory intensive or often not even possible.

The FDTD method makes use of Maxwell's equations in differential form, from which it can be seen that the change in electric field (E) over time is dependent on the change in magnetic field $(\mathrm{H})$ over space (curl) and the previous value of the E field at that location [15]: 


$$
\begin{array}{r}
\nabla \times \vec{H}=J+\frac{\partial \vec{D}}{\partial t}+\frac{\sigma \vec{D}}{\varepsilon} \\
D=\varepsilon \vec{E} \\
\therefore \nabla \times \vec{H}=J+\varepsilon \frac{\partial \vec{E}}{\partial t}+\sigma \vec{E}
\end{array}
$$

The magnetic field over time is dependent on the changes in electric field over space (curl) and the previous value of the $\mathrm{H}$ field at that location [15]:

$$
\begin{gathered}
\nabla \times \vec{E}=-\frac{\partial \vec{B}}{\partial t}-\frac{\sigma \vec{B}}{\mu} \\
H=\frac{B}{\mu} \\
\therefore \nabla \times \vec{E}=-\mu \frac{\partial \vec{H}}{\partial t}-\sigma_{H} \vec{H}
\end{gathered}
$$

Maxwell's equations are then discretized over space and time and used to calculate $\mathrm{E}$ and $\mathrm{H}$ fields. A total of six discrete equations are used to calculate each component of the $\mathrm{E}$ and $H$ fields $\left(E_{x}, E_{y}, E_{z}, H_{x}, H_{y}, H_{z}\right)$. These equations are used to calculate spatial fields in the time domain in leap frog form (one after the other) since they are co-dependent. The SAR is then calculated from the E-field via Equation 1.1, based on parameters of the medium. 
The discretized grid is created by dividing the model into discrete voxels (cubes) known as Yee cells (named after Kane Yee, who first described FDTD principles in a 1980 paper published in IEEE transactions on Electromagnetic Compatibility) as shown in Figure 2.12.

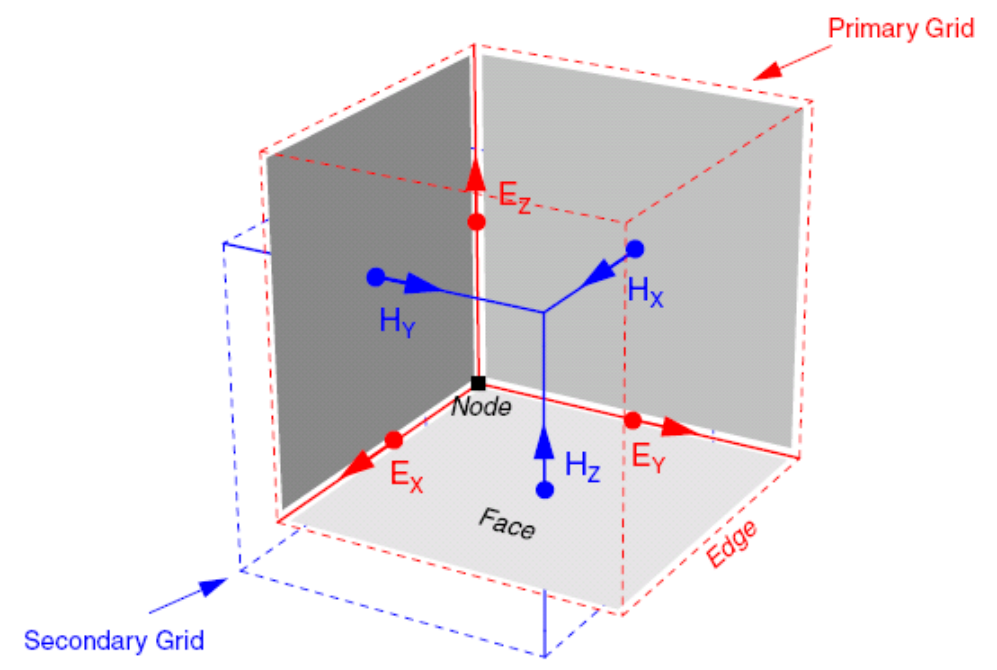

Figure 4.12: 3D Yee Cell with $E$ and $H$ field components [15]

The electric field components are assigned to the center of each edge of the cells, and the magnetic field components to the edge of each face of the cells [13]. For stability (a field resulting from an electromagnetic wave cannot be calculated in a cell before it actually reaches there) the simulation time step must satisfy the Courant-Friedrichs-Lewy stability criterion shown below [13].

$$
\Delta t<\frac{1}{c \sqrt{(1 / \Delta x)^{2}+(1 / \Delta y)^{2}+(1 / \Delta z)^{2}}}
$$

There are many highly advanced and developed commercially available FDTD simulators which implement the latter method; ITIS's SEMCAD X was used for this study [16]. 


\subsection{Experimental SAR Mapping Techniques}

Several methods may be used to generate an experimental SAR map of a phantom, including SAR probes, E-Field probes, thermographic method, and fiber optic thermometry. Takahiro Kawamura et al. made a SAR map of a solid cylindrical head model phantom using the thermographic method and obtained results with good agreement to within $10 \%$ between measured and calculated values [17]. Though this method is appealing in that it allows a high resolution picture of SAR distribution, it can only be applied to a solid phantom, and a thermographic image of the phantom is taken after RF heating actually takes place and the phantom is removed from the MRI, leaving room for error as temperature is not monitored while imaging. Generally, RF heating experiments are done in liquid phantoms and hence the thermographic method would not apply for most cases. Ulrich Katscher uses a new method known as electric properties tomography (EPT) to determine SAR via $\mathrm{B}_{1}$ (MRI RF field) mapping, but this requires a fully operational RF Coil (receive and transmit) [18]. It seems that the most cost-effective and potentially accurate method is fiber optic thermometry. Jallal makes use of this method to measure lead heating and finds that it is "effective yet prone to temperature probe noise and variations in the experimental setup" [19].

Herein, I have chosen to use fiber optic thermometry to map the SAR of an ASTM standard torso phantom in a 64MHz RF Quadrature Birdcage. While it provides low temperature resolution as compared to other methods, and is prone to temperature probe noise, it provides a direct measurement, versatility of use in various biological mediums, and is cost effective. 
The fiber optic thermometry method makes use of commercially available fiber optic temperature probes to monitor temperature in the biological medium at different points and translate the temperature rise to SAR via Equation 1.1: $S A R=c \frac{\Delta T}{t}$, where c is the specific heat of the medium and $\Delta \mathrm{T} / \mathrm{t}$ is the temperature change over a specified period of time. With enough temperature probes, we can get a relatively good picture of the SAR distribution in homogenous mediums. 


\section{METHODS}

The previous chapter discussed the importance of the characteristics of the RF $B_{1}$ field generated in the bore of an MRI. This was followed by an explanation of the use of phantoms as an experimental medium in which to get indications of potential heating in patients. Following this was an explanation of the FDTD method of simulation, for verification and understanding of field distributions generated in biomedical mediums. Finally, potential methods for measuring the SAR distribution in the phantom along with advantages and disadvantages were highlighted. Chapter 3 describes the tools developed to map the SAR distribution of a standard gel phantom, simulate the SAR distribution of a standard gel phantom, and finally compare the measured and simulated results. First, the phantom which was used for the experiments is described, followed by a brief overview of the fiber optic probes used, and finally an explanation of the automated MRI setup, created as a part of this thesis work.

\subsection{Phantom}

Experiments were performed in an ASTM standard torso phantom, of dimensions $42 \mathrm{~cm} \times 14 \mathrm{~cm} \times 65 \mathrm{~cm}$ with $1.27 \mathrm{~cm}$ walls made of acrylic [2]. The phantom was filled to 
$9 \mathrm{~cm}$ with a gel-like solution, which has conductivity $(\sigma) .49 \mathrm{~S} / \mathrm{m}$ and dielectric constant ( $\varepsilon$ ) of 78 (water is $\sim 80$ at DC), similar to electrical parameters of human muscle tissue. The phantom solution was prepared with deionized water, a concentration of $1.32 \mathrm{~g} / \mathrm{L}$ $\mathrm{NaCl}$, and $10 \mathrm{~g} / \mathrm{L} \mathrm{PAA}$ (polyacrylic acid), according to the procedures described in the ASTM standard [2].

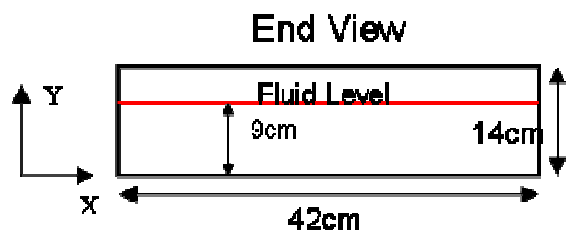

"Note: $1.27 \mathrm{~cm}$ acrylic walls around all sides, not shown
Side View

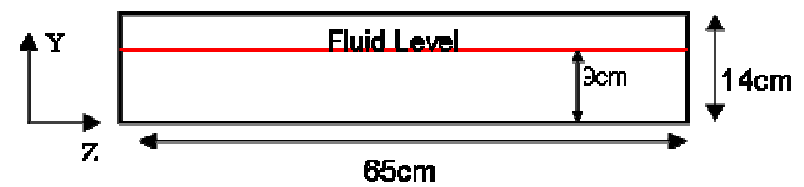

Figure 5.1: ASTM Torso Phantom Dimensions

A titanium rod (machined from ASTM B348 Grade 5 titanium) of $10 \mathrm{~cm}$ in length and $.35 \mathrm{~cm}$ in diameter (Figure 3.2 ), with two holes of $.1 \mathrm{~cm}$ diameter, $.05 \mathrm{~cm}$ from each end, is normally placed in the phantom to provide a stable thermal reference for normalization and indication of power being delivered to the phantom [19]. The Ti-Rod is used to normalize heating measurements between experiments for actual power delivered by the RF birdcage to the phantom. The ti-rod was kept in the setup as it is normally used for RF heating experiments, and hence it is important to map SAR in its region as well.



Figure 5.2: $10 \mathrm{~cm}$ Titanium Rod for normalization and reference

The Ti-Rod is centered at $\mathrm{X}=3 \mathrm{~cm}$ (from left wall of phantom), $\mathrm{Y}=2 \mathrm{~cm}$ (from floor of phantom), and $Z=21 \mathrm{~cm}$ (from top and bottom walls of phantom), as shown in Figure 3.3. 
A fiber optic temperature probe is placed in the hole in one end of the ti-rod, as shown below in Figure 3.4. This is a region of high SAR and results in high heating at the ends of the Ti-Rod (well out of the noise floor of the probes), hence making it an excellent choice for a reference object for RF heating measurements on metallic implants, if probe placement can be kept consistent. Though the ti-rod is routinely used for normalization of implant heating measurements (hence it was left in the setup), it was not used for normalization in this study; instead, the measurements made at any location in the phantom were normalized by the an attenuated (by a directional coupler) version of the output voltage of the RF amplifier, which can vary between experiments, delivering different amounts of power to the phantom.

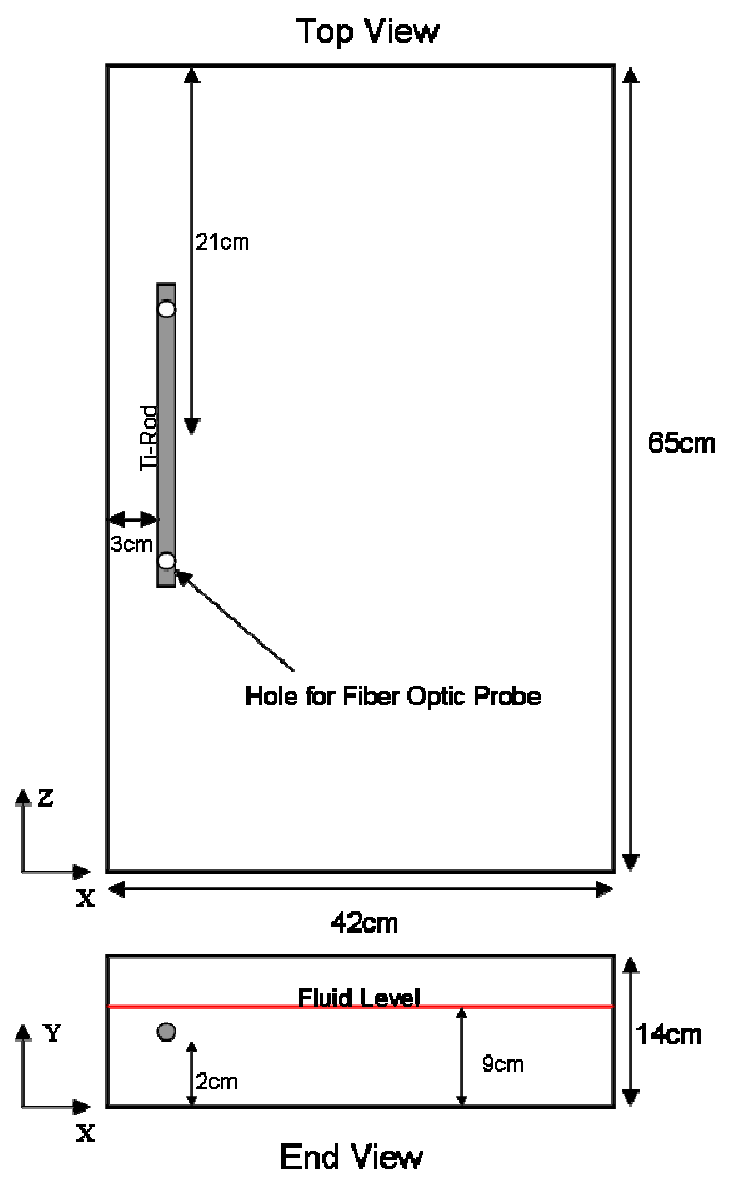

Figure 5.3: Ti-Rod Position in Phantom 


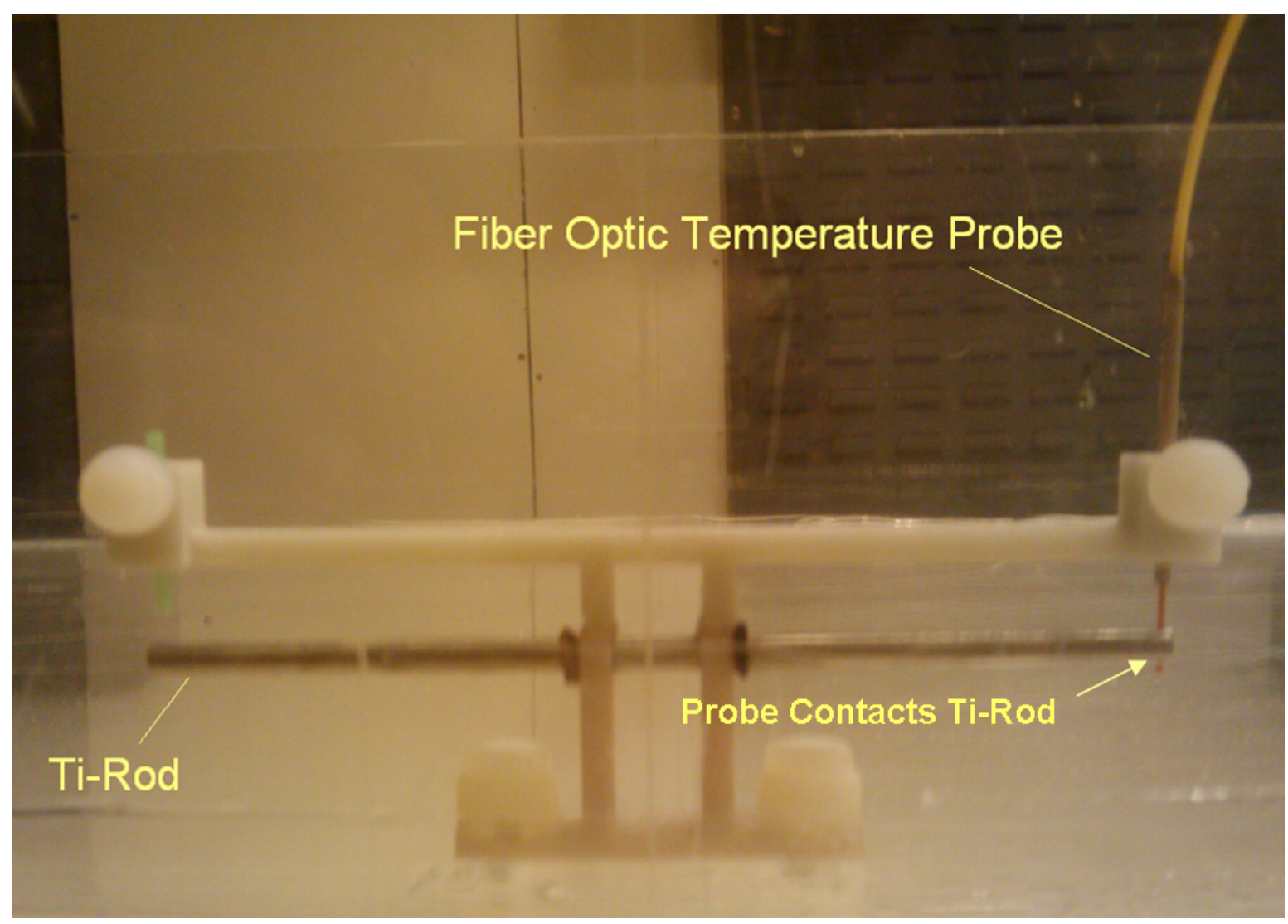

Figure 5.4: Ti-Rod in Phantom with fiber optic temperature probe

\subsection{Fiber Optic Thermometry}

Temperature measurements were made using custom fiber optic temperature probes (Neoptix, Quebec, Canada). Fiber optic probes were used over the conventional thermocouples, because unlike thermocouples, fiber optic temperature probes are not conductive and measurements would not be effected by coupling between electromagnetic fields and the measurement instrument itself. Fiber optic probes are often used in MRI environments when making phantom RF heating measurements of metallic implants for the latter reason. A sample setup is shown in Figure 3.5, for a single set of measurements with 14 Neoptix temperature probes. 


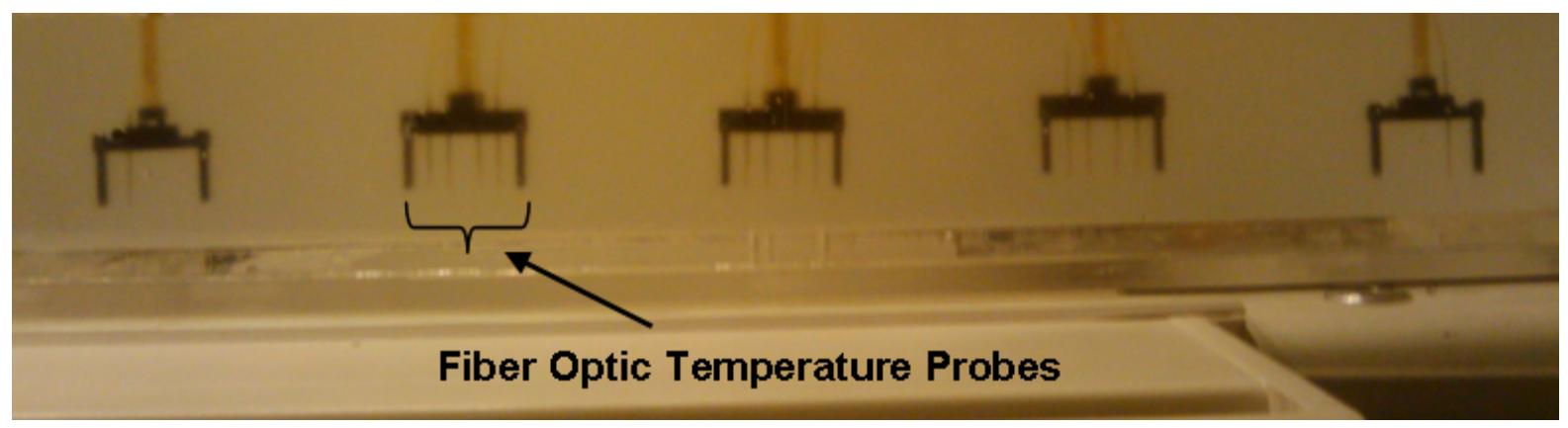

Figure 5.5: Experimental Setup - Single Spline Measurement 14 probes

The probes are designed to fit to the custom fixtures while also allowing the user to precisely place the sensitive element. A Neoptix Omniflex module was used to collect data and serially transmit to the computer, wherein a NI LabView automation program was implemented to collect, plot in real-time, and save the data.

\begin{tabular}{|c|c|}
\hline Model & Neoptix T1 Fiber Optic Temperature Sensor \\
\hline Temperature Range & -80 to +250 Degrees Celcius \\
\hline Materials & PTFE Teflon (protective jacket) \\
\hline Length & 1 meter \\
\hline Response Time & $500 \mathrm{~ms}$ \\
\hline Sensitive Element Size & 400 microns \\
\hline Accuracy & $.5^{\circ} \mathrm{C}$ \\
\hline
\end{tabular}

Table 1: Neoptix Fiber Optic Temperature Sensors Specification

\section{Temperature Sensitive Element}

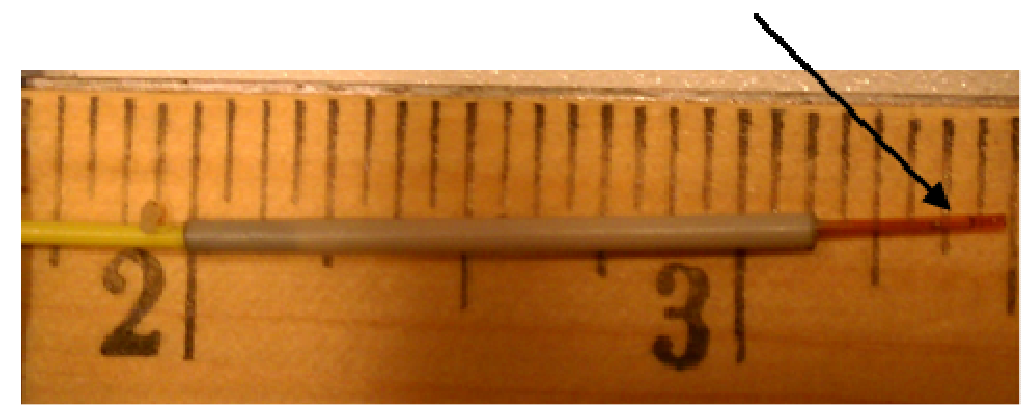

Figure 5.6: Custom Neoptix Fiber Optic Probe 


\subsection{RF Quadrature Birdcage}

A quadrature RF birdcage coil was used to generate the $64 \mathrm{MHz}$ field for a $1.5 \mathrm{~T}$ static magnet. As they are not relevant to RF heating experiments, the superconducting solenoid and gradient coils, were not included in the experimental setup.


Figure 5.7: 64MHz RF Quadrature Birdcage (looking into bore) used for measurements (left), 20kW RF Amplifier (right)

An Erbtec, three-stage $20 \mathrm{~kW}$ (peak pulsed power) RF Amplifier was used to amplify a $10 \mathrm{~Hz}, 5 \%$ duty cycle square-pulsed continuous wave RF signal generated by a Tektronix AFG3252 Function Generator. The square pulse and the $64 \mathrm{MHz}$ RF signal are fed to the amplifier, which effectively modulates the RF with the square wave gating signal and then amplifies it, as shown in Figure 3.8. 


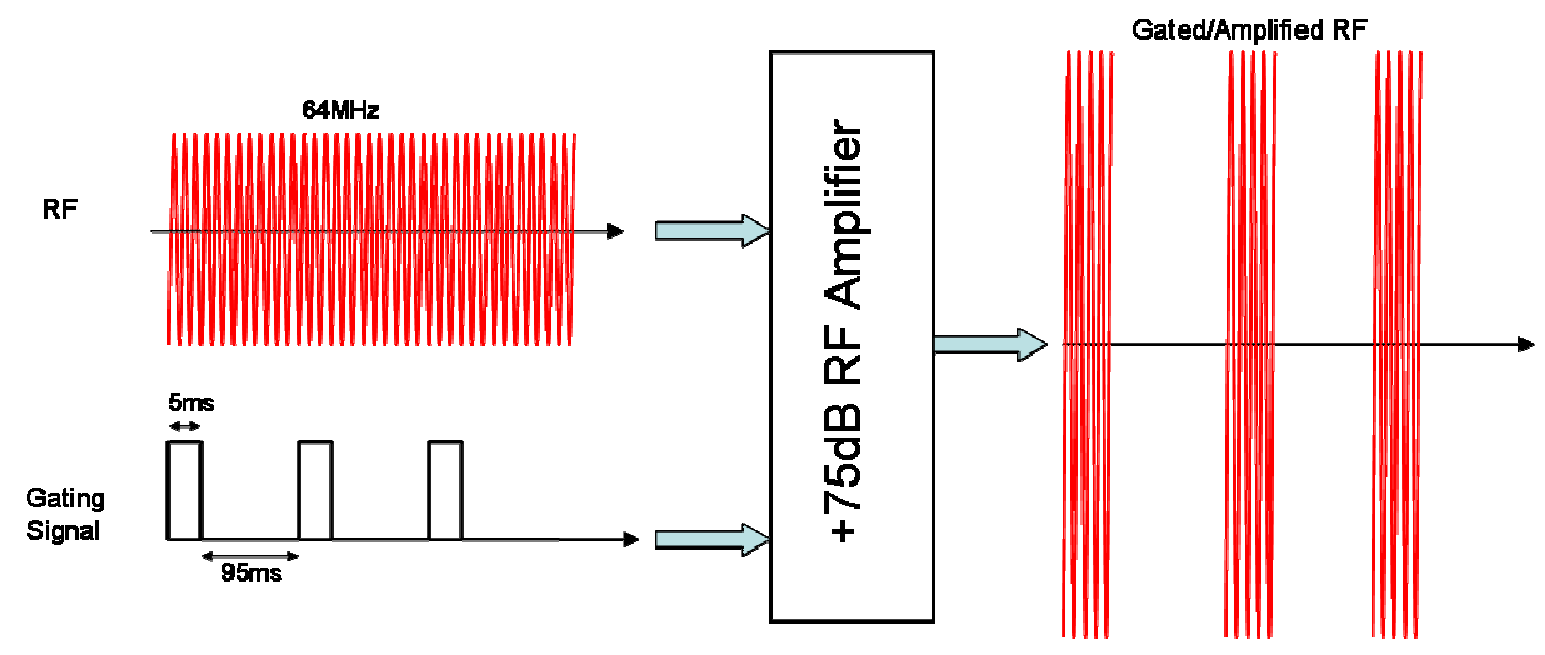

Figure 5.8: The function generator sources the RF amplifier with a gating signal and the RF signal.

The amplifier gates the RF signal, and amplifies it by $+75 \mathrm{~dB}$.

The power is controlled at the function generator, where the operator can adjust the amplitude of the ingoing RF signal via the automated LabView front panel (Figure 3.18). The amplified RF signal is then passed through a General Electric (GE) hybrid splitter which splits the signal and adds a 90 degree phase offset between the two signals.

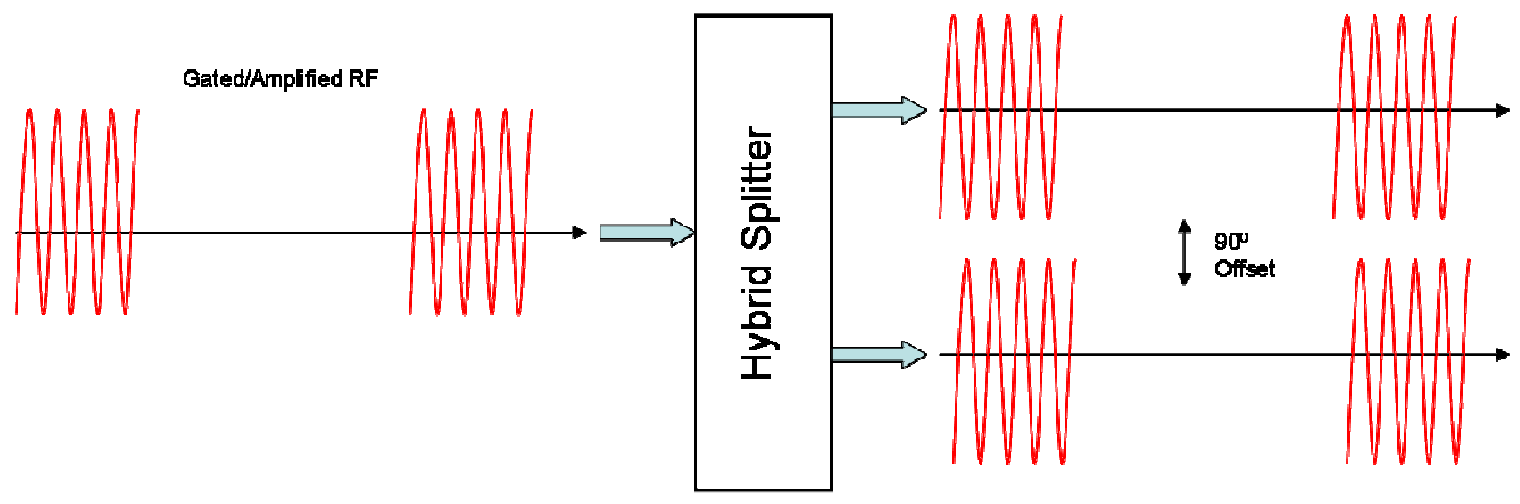

Figure 5.9: The amplified RF signal is passed through a Hybrid Splitter which splits the signal and adds a 90 degree phase offset between the two signals

The two orthogonal signals are then sent to two rungs of the RF birdcage coil (16 rungs total), which generates a circularly polarized $\mathrm{B}_{1}$ field as shown in Figure 3.10. 




Figure 5.10: The two orthogonal signals from the hybrid splitter are then sent to two rungs of the birdcage (which has a total of 16 rungs), and the birdcage generates a circularly polarized magnetic field $\left(\mathbf{B}_{1}\right)$.

An overview of the automated setup created for experimentation purposes is shown in Figure 3.11. The setup was created to streamline the experiments, and improve control, repeatability, and documentation done to conduct RF heating measurements in the birdcage. A Tektronix DPO3034 Oscilloscope was used to monitor power output from the RF Amplifier via a -50dB directional coupler. The Agilent E3644A power supply sources custom electronics, which are used to bypass software controls for enabling and disabling the RF amplifier. The Tektronix AFG3252 Function Generator sources the RF Amplifier with an RF and gating signal. 


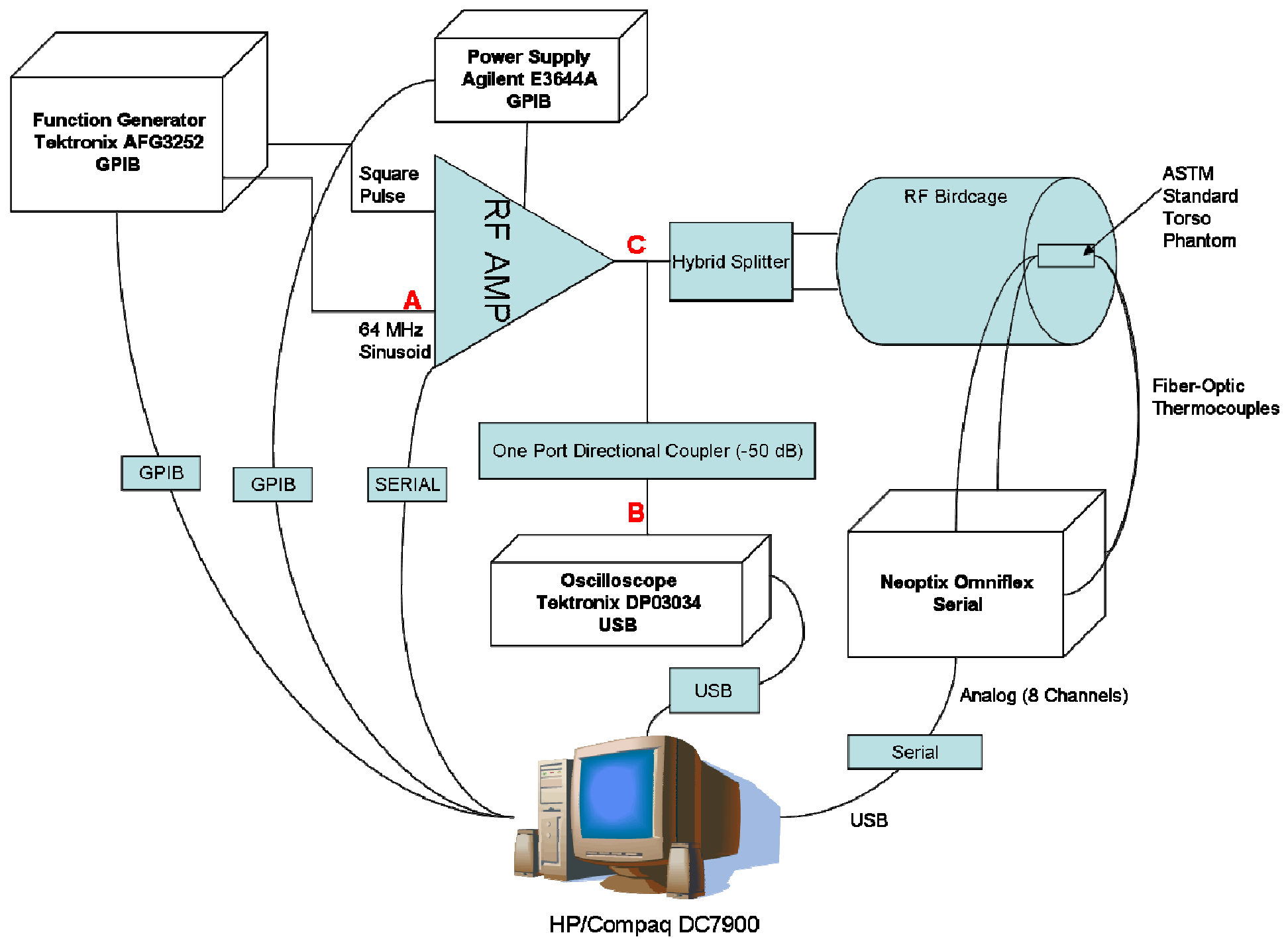

Figure 5.11: Automated MRI RF Heating Measurement Setup 


\subsection{Measurements}

Heating measurements were made with $\mathrm{RF}_{\text {input }}$ amplitude of $350 \mathrm{mVpp}$ (into $\mathrm{RF}$ amplifier) and an RF application time of 1800 s pulsed with a $10 \mathrm{~Hz}, 5 \%$ duty cycle square wave as shown in Figure 3.8. Table 2 below shows the relation of input power from the function generator to final $\mathrm{RF}$ amplifier output power to the birdcage. $T$ he $\mathrm{RF}_{\text {out }}$ power is $50 \mathrm{~dB}$ up from the directional coupler and $75 \mathrm{~dB}$ up from the $\mathrm{RF}_{\text {in }}$ signal. The power and $\mathrm{RF}$ application time were chosen to get reliable temperature measurements in the lowest SAR region of the phantom above the noise level of the custom Neoptix temperature probes $\left(\sim 0.5^{\circ} \mathrm{C}\right)$. The phantom was placed at the center of the birdcage, which gives relatively symmetric loading to the birdcage.

\begin{tabular}{|c|c|c|c|}
\hline $\begin{array}{c}\mathbf{R F}_{\text {in }} \text { (Figure 3.11, } \\
\text { Point A) }\end{array}$ & $\begin{array}{c}\text { Directional } \\
\text { Coupler (Figure } \\
\text { 3.11, Point B) }\end{array}$ & $\begin{array}{c}\mathbf{R F}_{\text {in } \text { Calculated }} \\
\text { Power (Figure 3.11, } \\
\text { Point A) }\end{array}$ & $\begin{array}{c}\text { Directional } \\
\text { Coupler Calculated } \\
\text { Power (Figure 3.11, } \\
\text { Point B) }\end{array}$ \\
\hline $350 \mathrm{mVpp}$ & $\sim 6.08 \mathrm{Vpp}$ & $\begin{array}{c}9681 \mathrm{~W} \text { peak pulsed } \\
\text { power }\end{array}$ & $\begin{array}{c}9238 \mathrm{~W} \text { peak pulsed } \\
\text { power }\end{array}$ \\
\hline
\end{tabular}

Table 2: Power Input to Birdcage for RF heating Measurements

A single measurement consisted of 14 temperature probes, conveniently placed along a straight line path (spline), parallel to the long edge of the phantom is shown in Figure 3.12. Temperature at each point was sampled at 5Hz. Figure 3.12, shows a measurement grid wherein measurements were taken at the crossings of individual dashed lines. A total of 210 point measurements were taken. The measurements were taken at three depths of $6.9 \mathrm{~cm}, 9.65 \mathrm{~cm}$, and $11.9 \mathrm{~cm}$ from the phantom floor. 


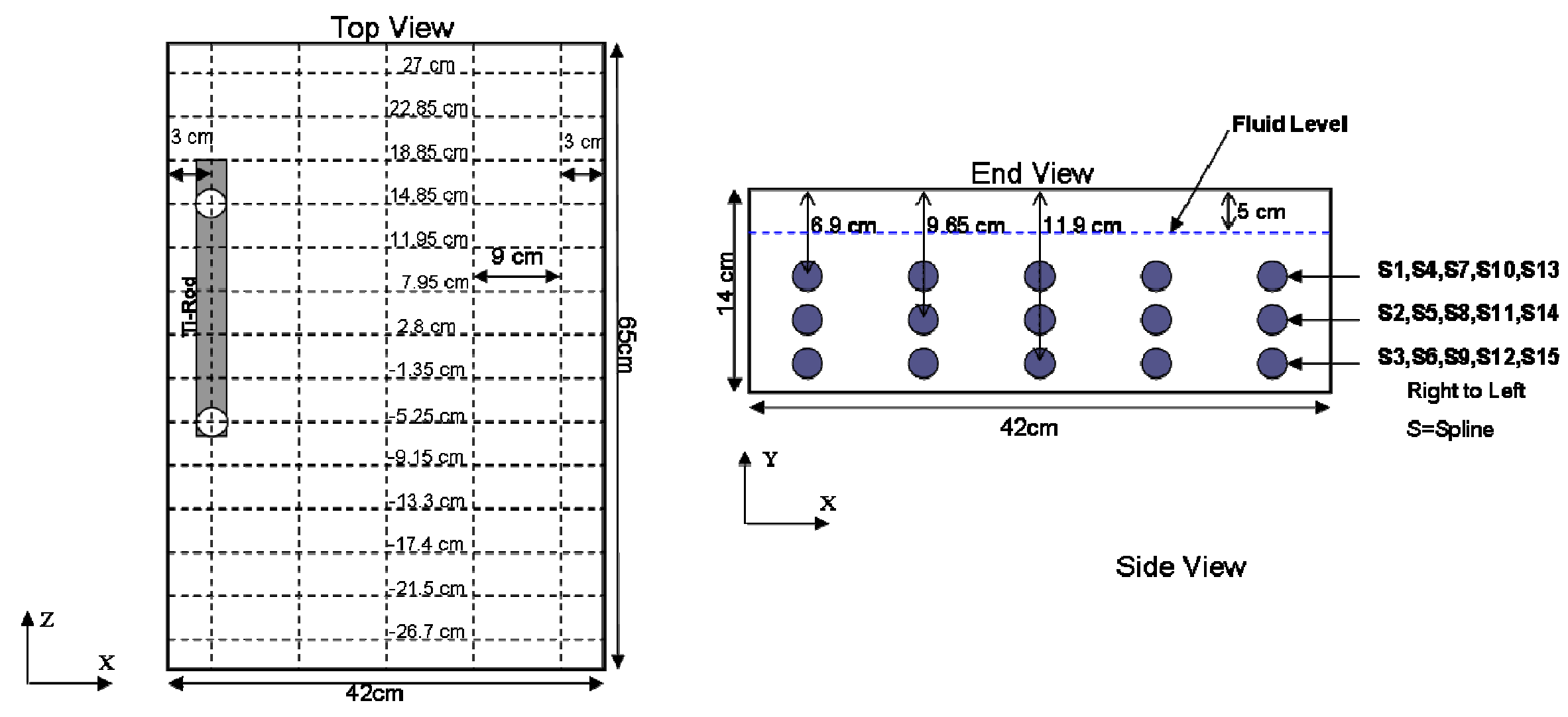

Figure 5.12: Measurement Grid (210 total measurement points 5x3x15) 
The required spatial resolution to determine curvature of SAR for each spline was determined via FDTD simulations. Figures 3.13-3.15 show the simulated SAR splines along each axis which require the greatest number of discrete point measurements to map. The highest order polynomial observed is a $4^{\text {th }}$ order polynomial which could easily be mapped with the number of probes available (14), hence I chose to map 14 points along the Z-axis, 5 across the $\mathrm{X}$-axis and 3 along the $\mathrm{Y}$-axis. After each RF heating measurement duration of 1800 s, the phantom mixture was stirred thoroughly to return the phantom to thermal equilibrium before continuing to the next experiment. The region around the ti-rod was stirred more vigorously, due to high heating in that area. The $1800 \mathrm{~s}$ measurement duration was preceded by a 30s startup time during which data is being logged to calculate the background temperature with the RF signal off and followed by a 30s settling time, wherein data is being logged where the phantom is cooling after RF exposure. It took approximately 1 hour to run a single experiment including setup time.



Figure 5.13: Simulated Spline along $X$-Axis requiring most points to characterize ( 4th order polynomial) 


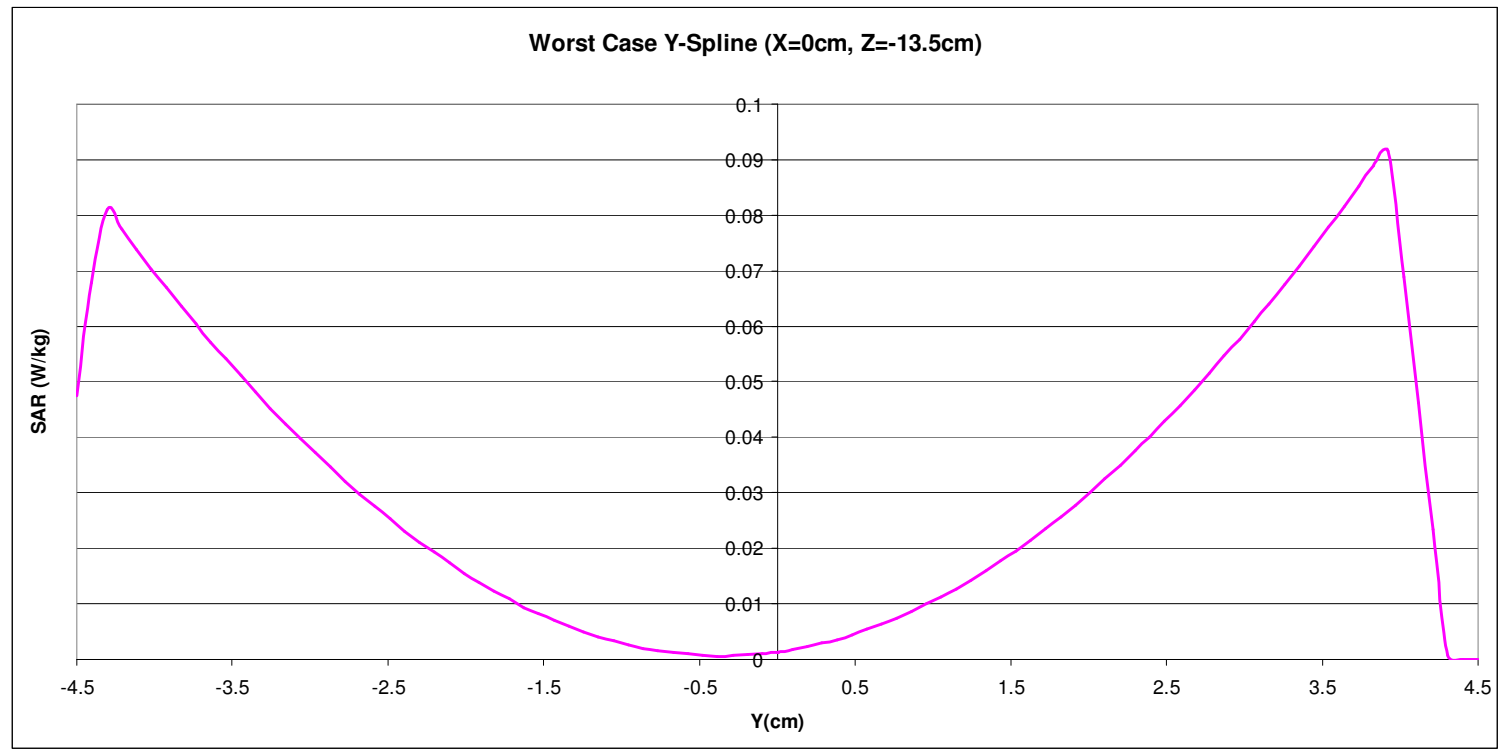

Figure 5.14: Simulated Spline along Y-Axis requiring most points to characterize (2nd order polynomial)

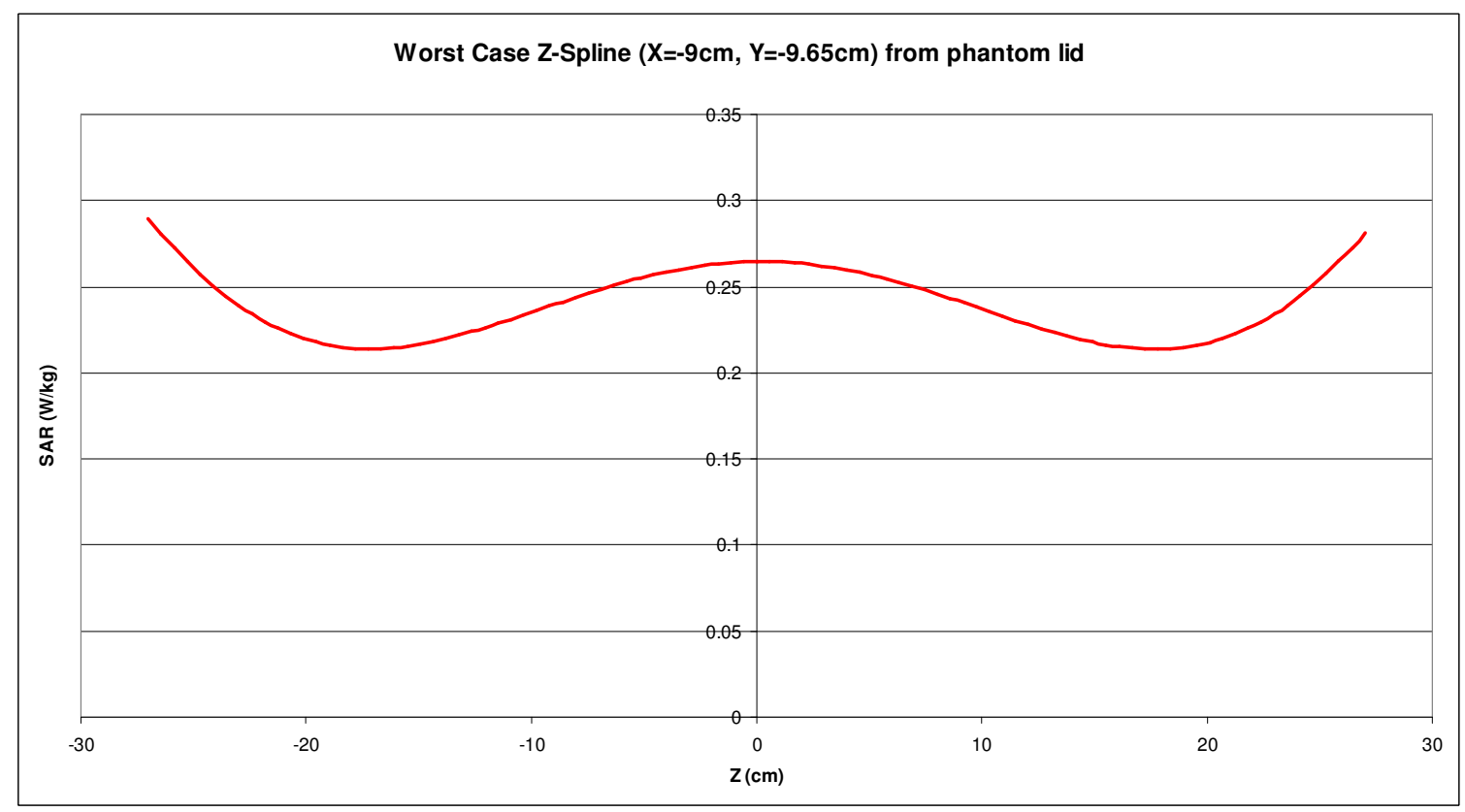

Figure 5.15: Simulated Spline along Z-Axis requiring most points to characterize (4th order polynomial) 
A set of experimental temperature plots vs. time for a single spline are shown in Figure 3.16. Probe $1\left(\mathrm{P} 1_{\mathrm{M} 1}\right)$ is attached to the end of the ti-rod (reference object) as shown in Figure 3.3, and the last probe $\left(\mathrm{P}_{\mathrm{M} 4}\right)$ is recording ambient temperature in the room which is not relevant for this study as the measurements were made in a well insulated phantom. The ambient temperature probe is dangling in the room, therefore any spikes or unusual measurements are a result of the probe being tampered with. Refer to the appendix for temperature plots for each spline. As expected, a linear rise in temperature is observed at every point (except ti-rod and ambient), and SAR can be calculated by taking the slope of these curves and multiplying by the specific heat capacity of the medium. A greater temperature rise would give us more confidence in the measurements as this would bring the measurements out of the noise range of the temperature probes. 

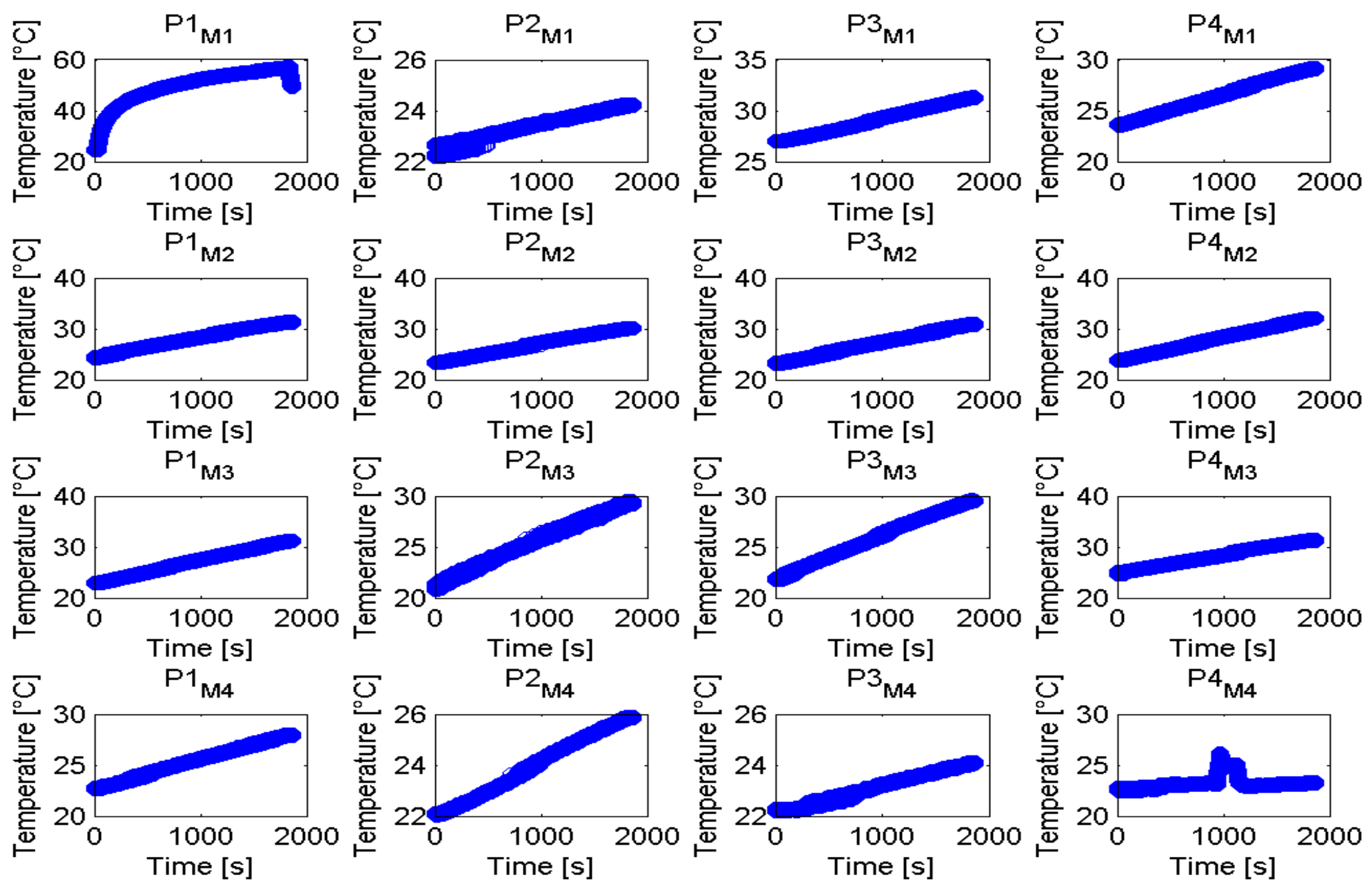

Figure 5.16: Temperature vs. Time for a Spline 1 (14 points along Z-axis) starting from P2 $2_{\mathrm{M} 1}$ (Probe 2 Module 1 ) to P3 ${ }_{\mathrm{M} 4}$ (Probe 3 Module 4 ). P1 $1_{\mathrm{M} 1}$ is the attached to the titanium rod, and $\mathrm{P4}_{\mathrm{M} 4}$ is ambient temperature, both of which are irrelevant for this study. 


\subsection{Automation}

The measurement system was automated using National Instruments LabView. As shown in Figure 3.11, several methods of communication were utilized to control various instruments. The Neoptix system is controlled via serial communication, the Power Supply via GPIB, the Function Generator via GPIB, the oscilloscope via USB, and the RF Amplifier via serial communication. Figure 3.17, shows the front panel through which the user can control all of the instruments, and monitor real-time displays of temperature probe readings. The automated system, essentially allows the user to start up the amplifier without having to switch to an external program, control all relevant parameters of the instruments, automatically log relevant setup parameters in a setup file and log temperature data in another file. The automated system leaves little room for operator error, and tracks several important system variables, improving repeatability of measurements. 


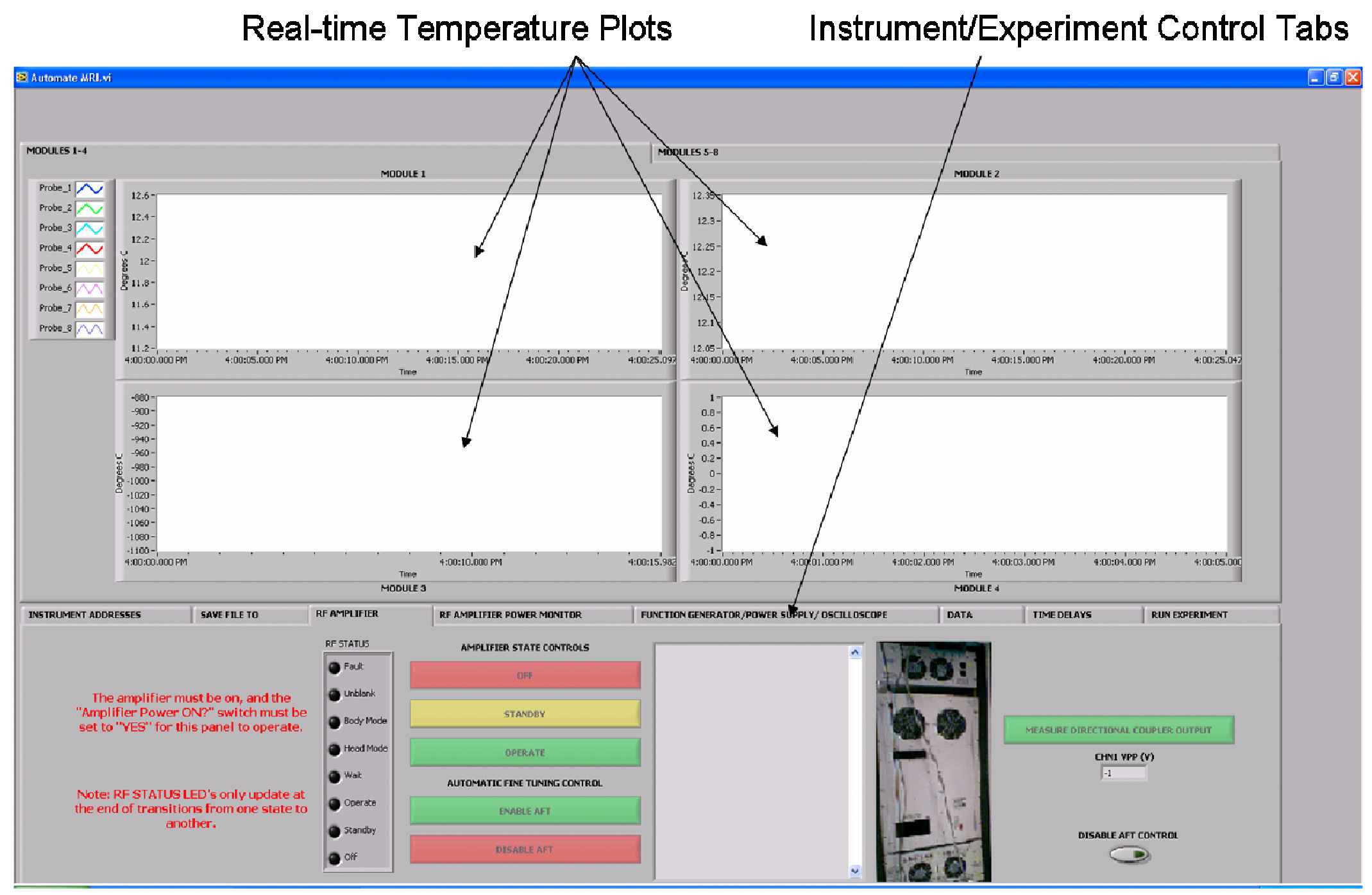

Figure 5.17: MRI RF Heating Experiment Automation Front Panel (LabView) 
To measure repeatability, Spline 3 (Figure 3.12) was re-measured five times, completely removing the probes from the setup (removing from fixtures and original positions), stirring the fluid, and repositioning the probes again. As shown in Figure 3.18, the standard deviation in SAR measurements was kept below $~ 36 \%$, along Spline 3. The standard deviation remained below $15 \%$ for high SAR regions (towards center of spline) which are of most interest for experiments. Though this may seem high at first glance, it is a relatively good measurement, considering that there are so many factors which can contribute to loss of repeatability in measurements (probe positioning, power delivered to birdcage, phantom positioning, changes in fluid properties, operator error, etc.), of which the dominating factor is likely probe positioning.

\section{Repeatability Analysis}



Figure 5.18: Five repeat SAR measurements were taken of Spline 3 (14 point measurements). The standard deviation in the SAR measurements remained below $36 \%$ and improved to below $15 \%$ for high SAR regions towards the center of the spline. 
The temperature measurements recorded for spline 3 ranged from rises of $\sim .6^{\circ} \mathrm{C}$ to $\sim 3^{\circ} \mathrm{C}$. This is a good range in which to measure repeatability as it gets very close to the minimum rating of the fiber optic temperature probes $\left(.5^{\circ} \mathrm{C}\right)$. The majority of the error is a result of our lacking temperature measuring capability at lower temperature rises, hence it may not be a good indication of repeatability at points with lower temperature rises, whereas at points with higher temperature rises, the variability may be attributed to operator error, positioning of probes, and less likely to equipment inaccuracy 


\section{FDTD SIMULATIONS}

This section describes the FDTD simulation setup for which the background was explained in section 2.3. The simulation setup was designed to mimic an ideal birdcage setup hence many of the parameters and models may not be an exact replicate of the experimental setup, but provide an ideal representation of the types of fields which are expected from an RF birdcage. This enables us to compare measurements to the idealized model. ITIS's SEMCAD X was used to model and run all simulations.

A 16 rung quadrature birdcage with an RF shield was modeled in SEMCAD (Figure 4.1). The RF shield had a diameter of $83.5 \mathrm{~cm}$ and was modeled as a perfect electrical conductor (PEC). The birdcage was $75 \mathrm{~cm}$ in diameter, and each leg was also modeled as a PEC. Each leg was fed with an ideal current source, with a $22.5^{\circ}$ phase offset between each source, to generate a circularly polarized homogenous magnetic field $\left(B_{1}\right)$ as shown in Figure 2.9. In an actual quadrature birdcage coil, the sources are not ideal and there are two feed points at rungs $90^{\circ}$ apart, each of which is fed with an RF signal $90^{\circ}$ apart from the other, and capacitors along the circular rim are designed to provide the rest of the phase offsets between each rung, while also being utilized to tune the coil to the desired frequency of operation, which in this case is $64 \mathrm{MHz}$. 


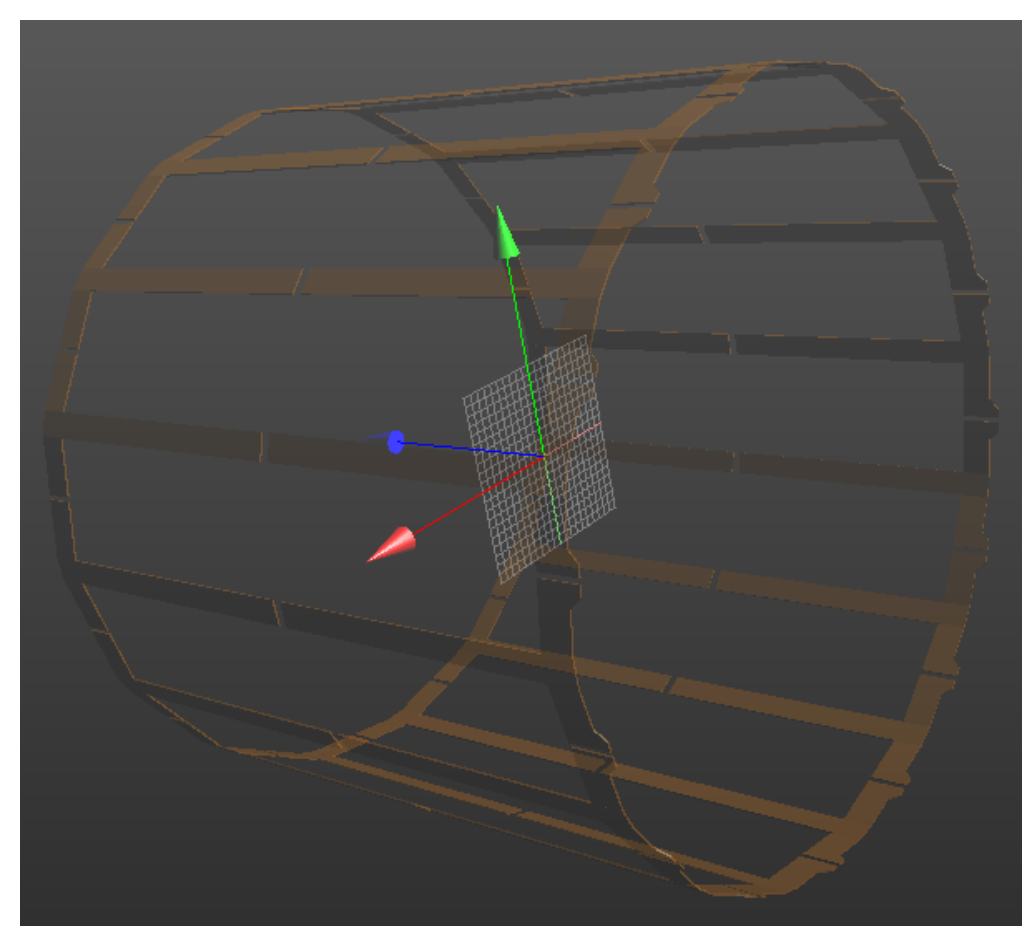

Figure 6.1: 16 Rung RF Quadrature Birdcage Coil Simulation Model

The phantom was modeled with dimensions $42 \mathrm{~cm} \times 9 \mathrm{~cm} \times 65 \mathrm{~cm}$. The acrylic enclosure was left out of the model as it was assumed that it would not have a significant impact on results unless it was very close to the edge of the phantom, where measurements are not taken to avoid edge effects. The model of the phantom was also only $9 \mathrm{~cm}$ in depth as compared to the experimental phantom which is $14 \mathrm{~cm}$ in depth but is filled to $9 \mathrm{~cm}$. The phantom material was modeled as a dielectric with relative permittivity of 78 , conductivity $.49 \mathrm{~S} / \mathrm{m}$, and density $1000 \mathrm{~kg} / \mathrm{m}^{3}$, which are the same parameters as the actual phantom.

The titanium rod (ti-rod), often used for normalization was also included in the simulation, as it is normally included in experimental setups. The ti-rod is modeled as a PEC, $10 \mathrm{~cm}$ in length, and $.3 \mathrm{~cm}$ in diameter. Due to its small size relative to the simulation, the Huygens box method had to be used to get timely simulation results. 
When simulating very fine resolution objects, it is important to grid them appropriately (finely). When these objects are part of a larger simulation model (RF birdcage), gridding so finely can result in extremely long simulation times and the Huygens box method can be used to avoid this. In the Huygens method, the entire model is simulated without the ti-rod (fine resolution object); thereafter a small portion of the simulation around the tirod (where the surroundings are immediately effected), is simulated with applied fields at the Huygens box which are determined from the overall simulation. The complete simulation model is shown below in Figure 4.2.

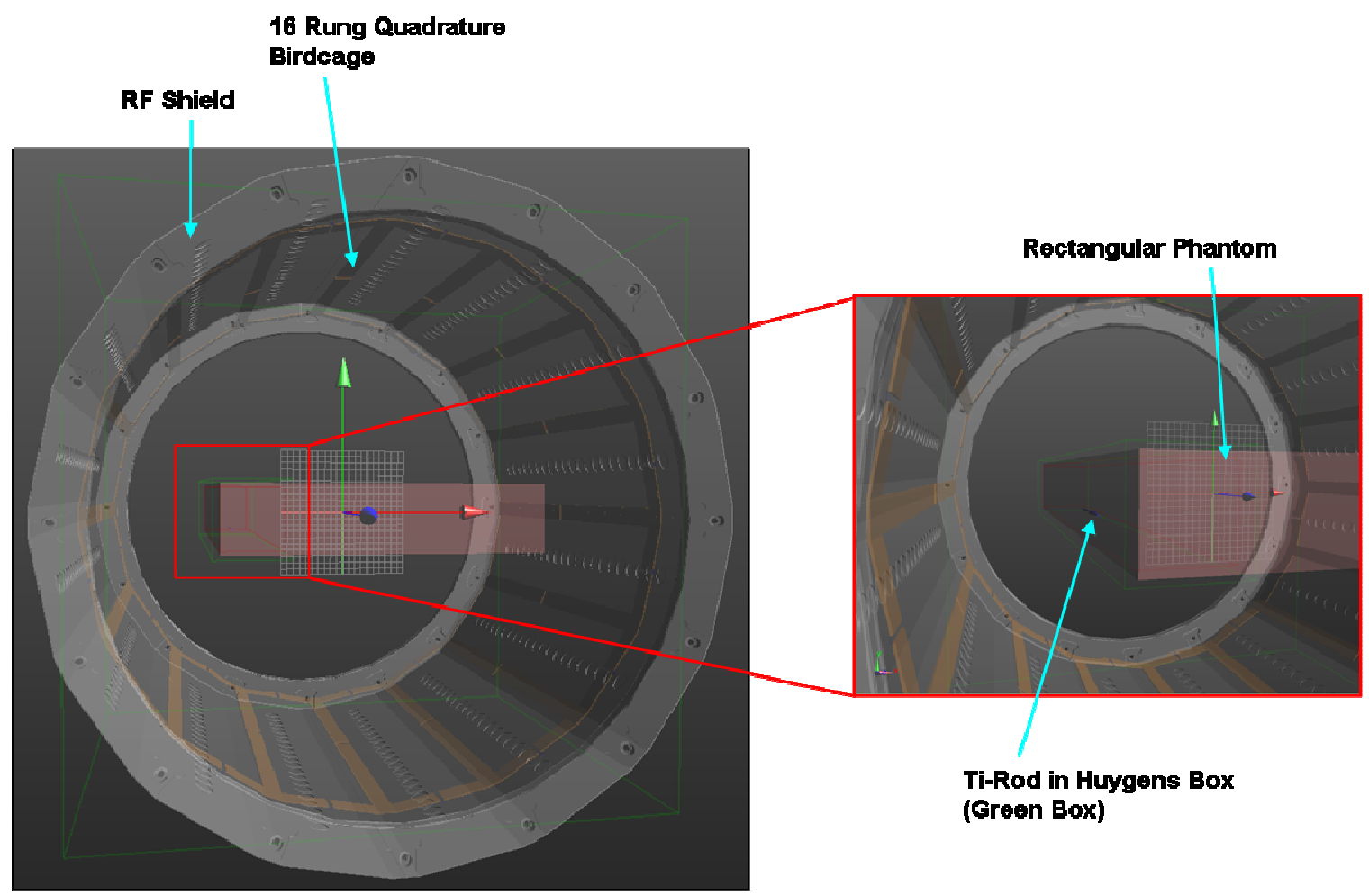

Figure 6.2: SEMCAD FDTD Simulation Setup with Ideal RF Quadrature Birdcage, ASTM Standard Phantom, and Titanium Rod Reference Object 


\section{RESULTS AND ANALYSIS}

This section presents the measured SAR map, the simulated SAR map of the phantom, and a comparison of the two. Furthermore, an analysis of the fiber optic method as a means to make these measurements, and its accuracy is included.

For each measurement point, a SAR was calculated and normalized to the directional coupler voltage squared, to account for any differences in power between experiments.

$$
S A R_{\text {norm }}=\frac{c \times \Delta T / t}{V_{D C}^{2}}
$$

Several regions of the temperature curves were used to calculate SAR, ranging from the start to the end of the RF application. This gave an indication of the effect that time of RF application had on the SAR measurements and their accuracy as compared to simulated results. Figures 5.1-5.3 show false color plots of the measured SAR distribution. In general, the SAR was highest near the walls of the phantom and weakest near the center as expected based on previous results in literature and electromagnetic theory in which eddy currents in the conductive media increase with object radius. One can also see the sharp peaks in SAR along the ti-rod at $\mathrm{Y}=11.9 \mathrm{~cm}$ from the phantom lid in Figure 5.1. All analysis was done in MATLAB, except for the FDTD simulation. 


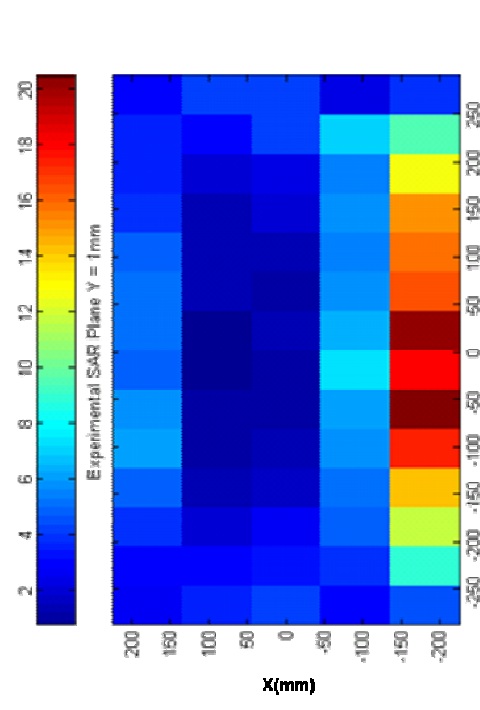

(A)



(B)



(C)

Figure 7.1: Spatial distribution of induced SAR (not normalized) within the phantom liquid in GE 64 MHz quadrature birdcage coil. Local temperature was measured with fiber optic temperature probes. $S A R$ is in units of $W / \mathrm{kg}$, Note different color scaling between subfigures. $Y=6.9 \mathrm{~cm}(A)$, $9.65 \mathrm{~cm}(B)$, and $11.9 \mathrm{~cm}(\mathrm{C})$ from phantom lid

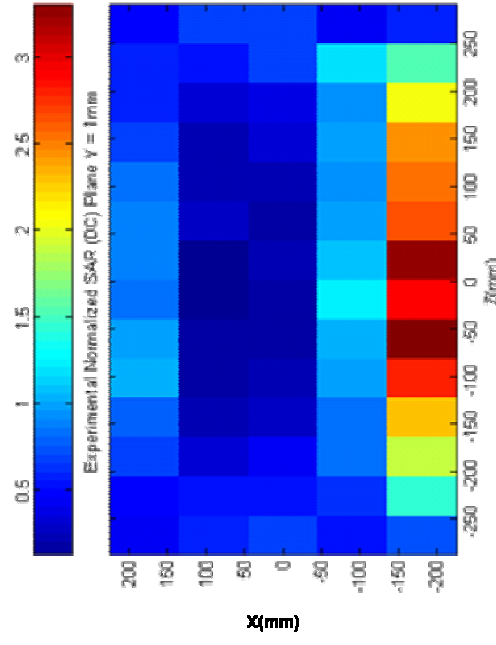

(A)

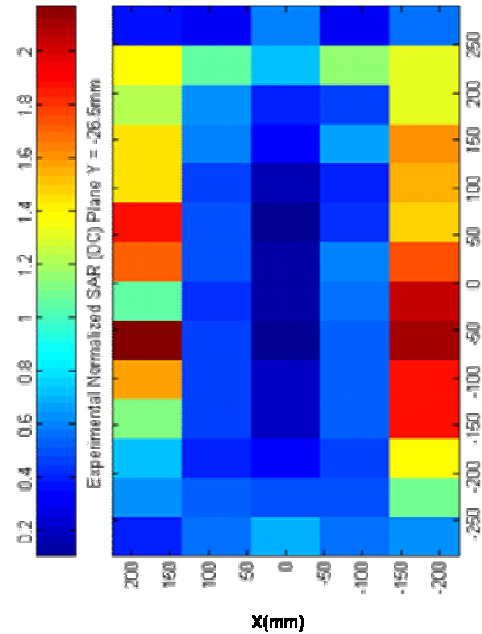

(B)

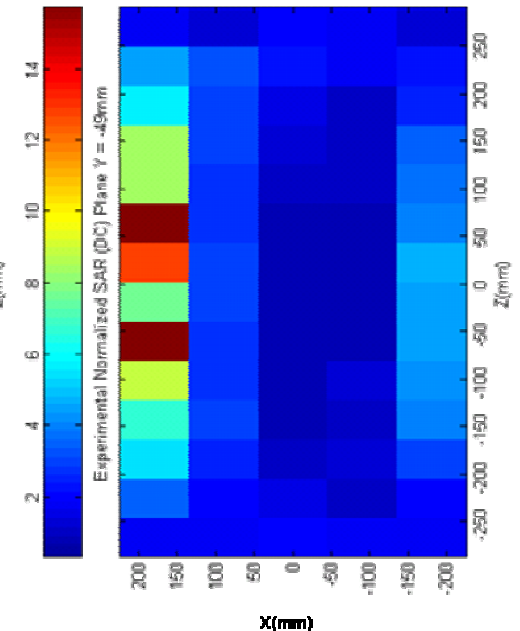

(c)

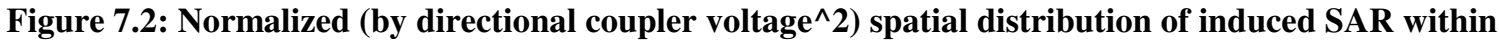
the phantom liquid in GE $64 \mathrm{MHz}$ quadrature birdcage coil. Local temperature was measured with fiber optic temperature probes. Normalized Units of $\mathrm{W} / \mathrm{kg} / \mathrm{V}^{2}$. Note different color scaling between subfigures. $Y=6.9 \mathrm{~cm}(\mathrm{~A}), 9.65 \mathrm{~cm}(\mathrm{~B})$, and $11.9 \mathrm{~cm}(\mathrm{C})$ from phantom lid 


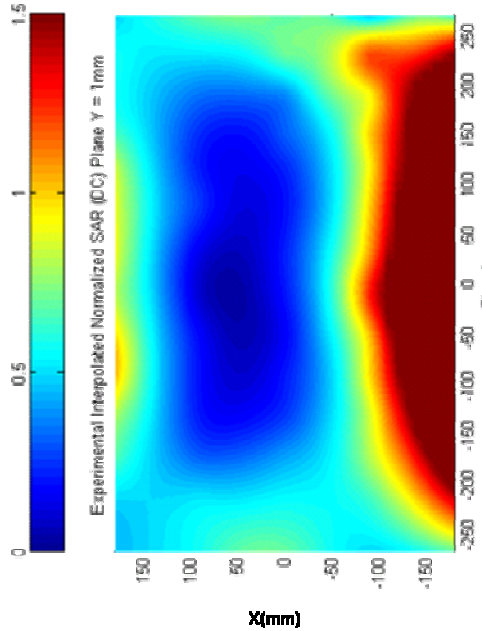

(A)
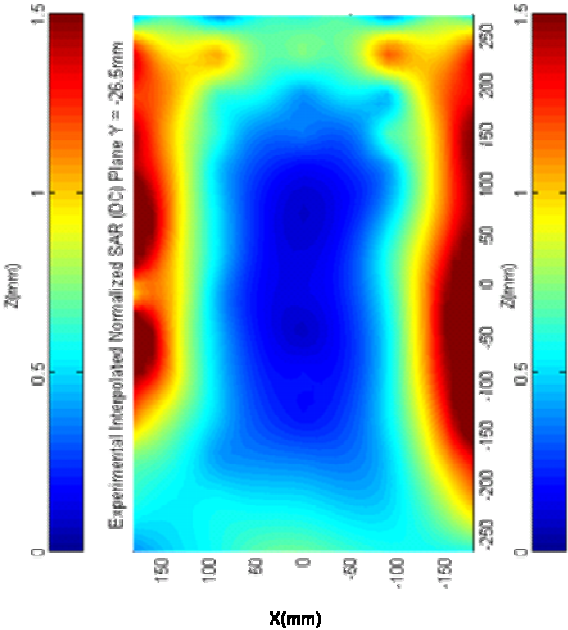

(B)

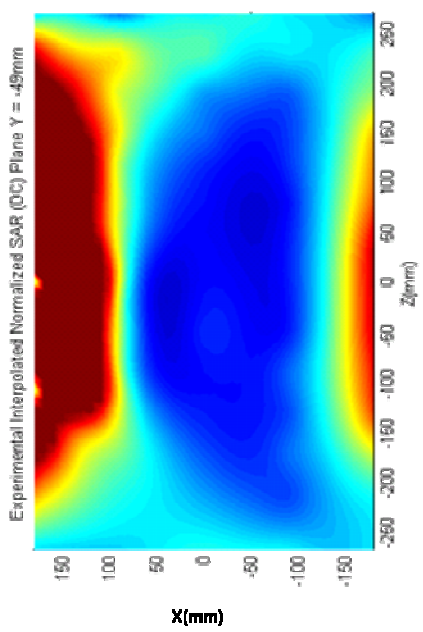

(C)

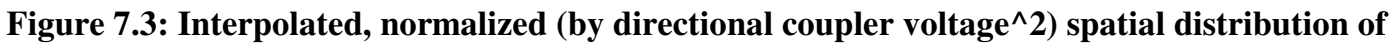
induced SAR within the phantom liquid in GE 64 MHz quadrature birdcage coil. Local temperature was measured with fiber optic temperature probes. Units of $W / \mathrm{kg} / \mathrm{V}^{2} . \mathrm{Y}=6.9 \mathrm{~cm}(\mathrm{~A}), 9.65 \mathrm{~cm}(\mathrm{~B})$, and $11.9 \mathrm{~cm}(\mathrm{C})$ from phantom lid

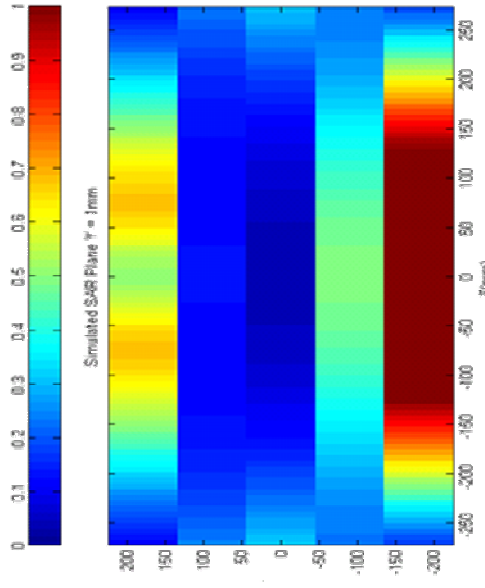

$x(m \mathrm{~mm})$

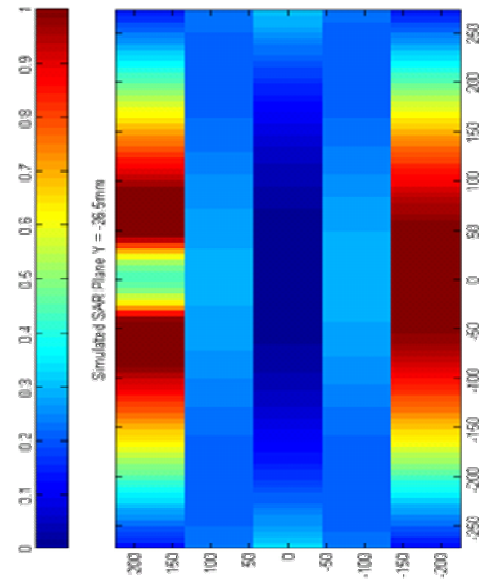

$\mathbf{x}(\mathbf{m m})$



$x(m m)$

(C)

Figure 7.4: Simulated spatial SAR distribution $(\mathrm{W} / \mathrm{kg})$ with Ti-Rod. $Y=6.9 \mathrm{~cm}(\mathrm{~A}), 9.65 \mathrm{~cm}(\mathrm{~B})$, and

$$
11.9 \mathrm{~cm}(\mathrm{C}) \text { from phantom lid }
$$

The Least Mean Square method (LMS) was used as a metric to choose a scaling factor $(\eta)$ between measured and simulated results. 


$$
\begin{gathered}
\eta=\frac{\sum_{i, j, k} S A R_{\text {sim }}(i, j, k) \times S A R_{\text {meas }}(i, j, k)}{\sum_{i, j, k} S A R_{\text {meas }}^{2}(i, j, k)} \\
\text { error }^{2}=\frac{1}{i \times j \times k} \sum_{i, j, k}\left(\eta S A R_{\text {meas }}(i, j, k)-S A R_{\text {sim }}(i, j, k)\right)^{2} \\
\text { error }=\sqrt{\frac{1}{i \times j \times k} \sum_{i, j, k}\left(\eta S A R_{\text {meas }}(i, j, k)-S A R_{\text {sim }}(i, j, k)\right)^{2}} \\
\text { Signal }=\sqrt{\frac{1}{i \times j \times k} \sum_{i, j, k}\left(S A R_{\text {sim }}(i, j, k)\right)^{2}} \\
\% \text { error }=\frac{\text { error }}{\text { signal }} \times 100
\end{gathered}
$$

As shown in Equation 5.3, mean square error of measured results as compared to ideal simulated results, is calculated by taking the average of the difference between the measured value multiplied by an unknown scaling factor and the simulated value. Thereafter we take the square root to get the mean error, and take its derivative with respect to error, leaving us with Equation 5.2, the solution for the least mean square error scaling factor.

To get a measure of percent error over the entire measurement/simulation space, the signal power is calculated from simulation results as shown in Equation 5.5 and error is determined by dividing the error by the total signal power. This metric is used throughout the analysis to determine the similarity or differences between simulated and measured. 
The error in SAR between measured and simulated results (calculated using equations 5.4-5.6) for the 15 splines is $87 \%$, the majority of which is a result of Spline 15 (nearest ti-rod, where gradients are high). Figure 5.5 shows the measured (pink) and simulated (blue) SAR results for Splines 1-15. If we remove Spline 15, we can see that the results match much better with an error of $24.05 \%$ (Figure 5.6). Furthermore, if all splines on the Ti-rod Y-Z plane (Splines 13-15) are removed from the results, the error becomes $21.09 \%$. 

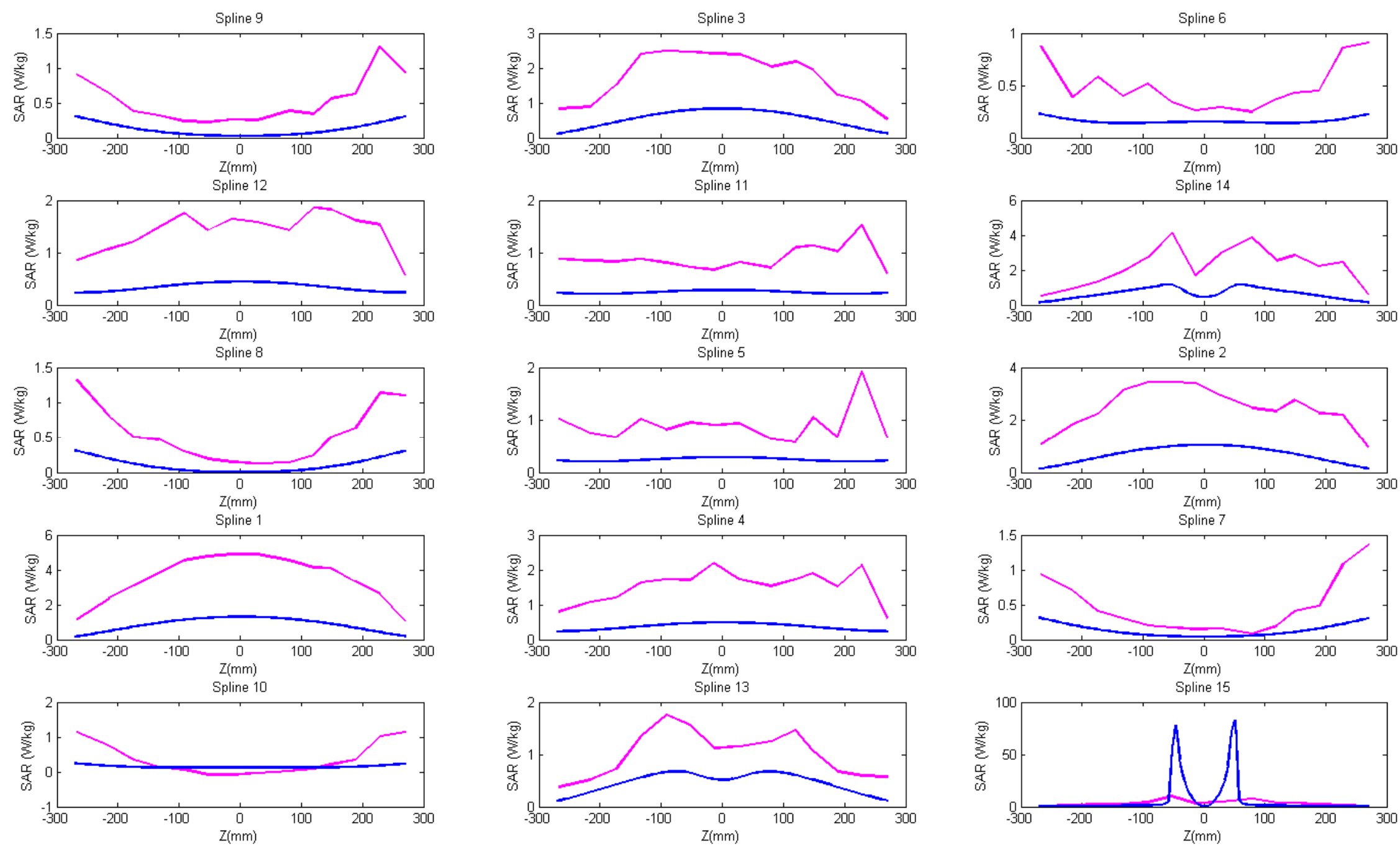

Figure 7.5: Measured (pink) vs. Simulated (blue) SAR Splines 1-15. $\eta=9.7117$, Error $=87 \%$ 

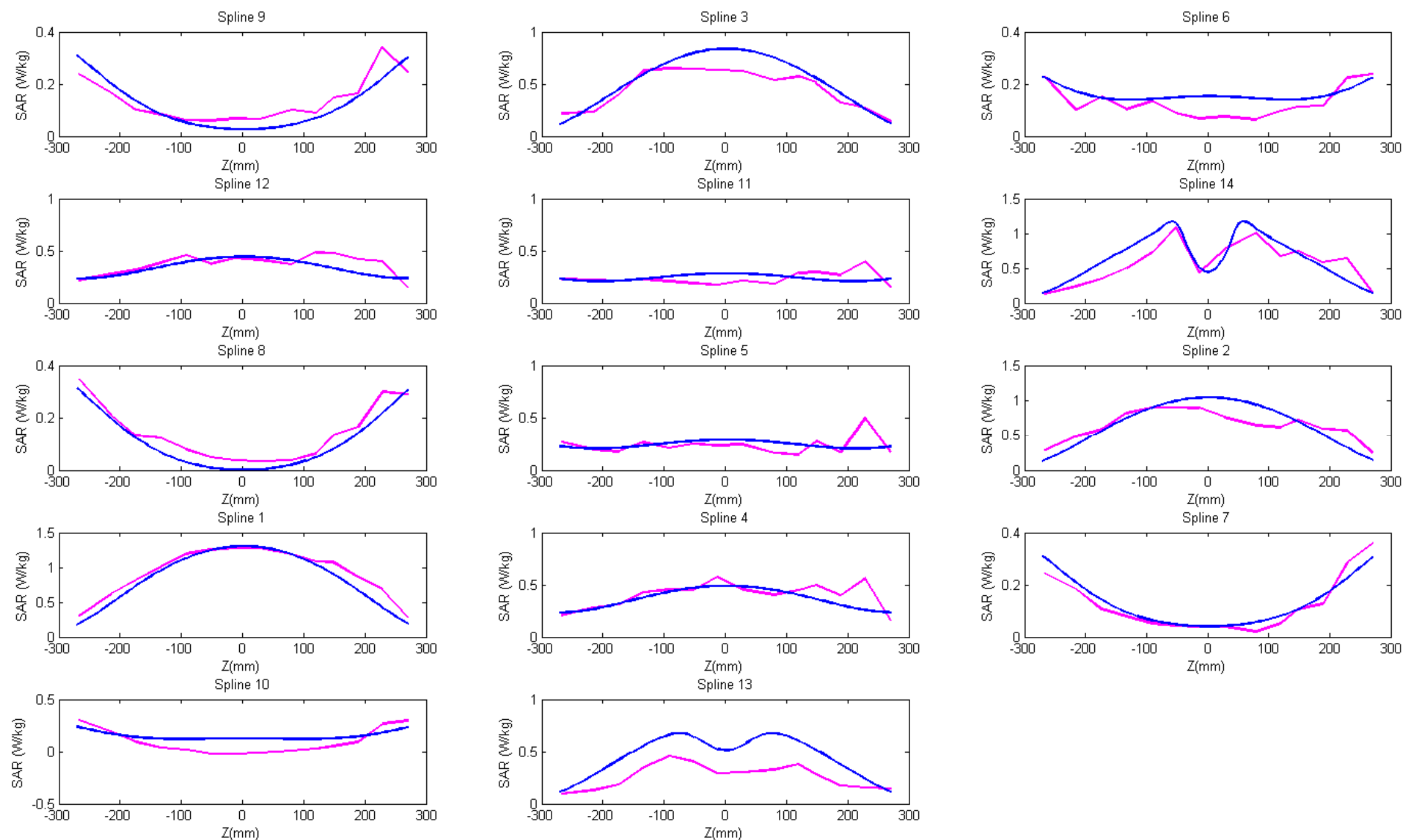

Figure 7.6: Measured (pink) vs. Simulated (blue) SAR Splines 1-14. $\boldsymbol{\eta}=\mathbf{2 . 5 5}$, Error $=\mathbf{2 4 . 0 5 \%}$ 
The regions in the phantom of most concern are regions with high SAR, as that is generally where objects are placed for RF heating measurements. In the high SAR region where experiments are normally performed (along Spline 1), an error of $10.7 \%$ in SAR, was obtained as illustrated in Figure 5.7

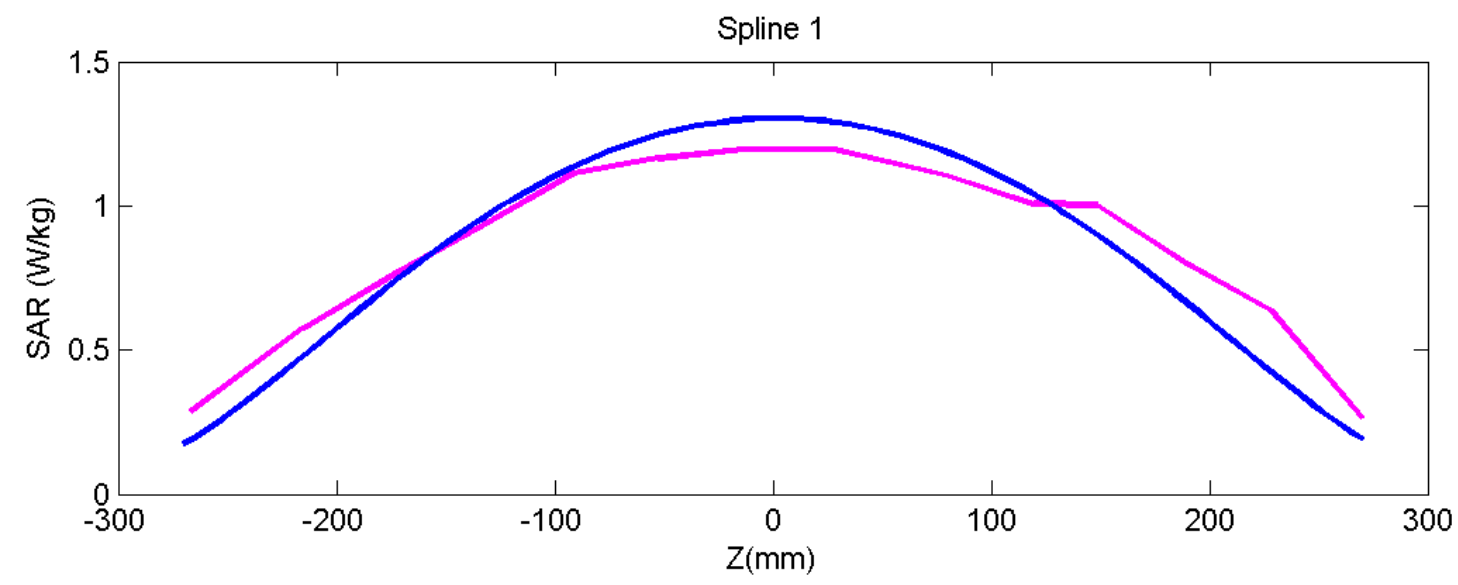

Figure 7.7: Spline 1 (high SAR region) SAR Meas. (pink) vs. Simulated (blue) $\boldsymbol{\eta}=\mathbf{2 . 3 6}$, Error $=\mathbf{1 0 . 7 \%}$

Figure 5.8 shows the spatial distribution of error between SAR measured and simulated. The error is much greater towards the center of the phantom and decreases when approaching the walls. This is a result of low SAR near the center of the phantom, which causes inaccurate temperature measurements as they are very low and very close to the noise level of the fiber optic probes $\left(.5^{\circ} \mathrm{C}\right)$. 


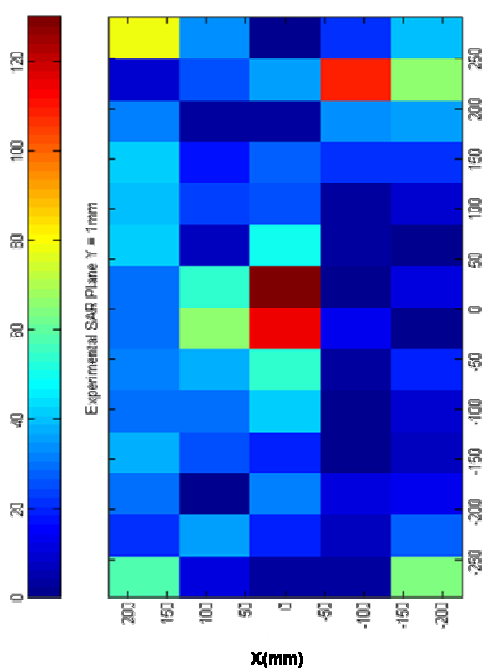

(A)

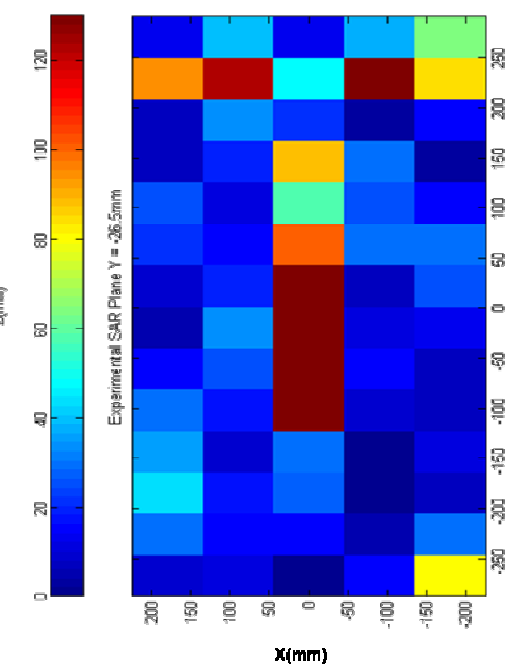

(B)

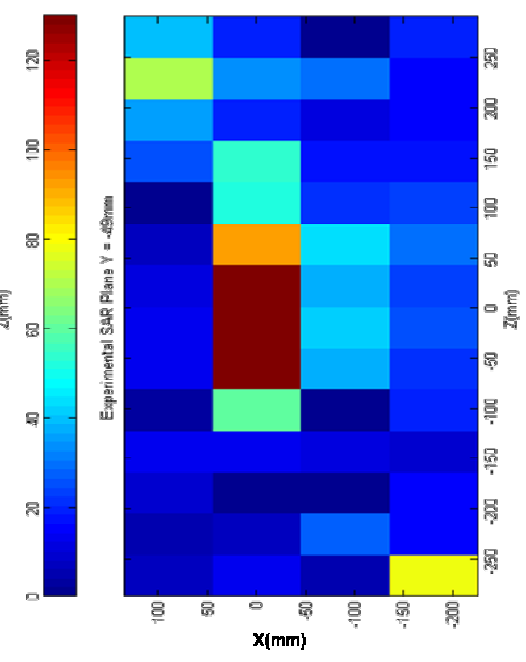

(c)

Figure 7.8: Error distribution between Simulated and Measured SAR. $Y=6.9 \mathrm{~cm}(A), 9.65 \mathrm{~cm}(B)$, and $11.9 \mathrm{~cm}(\mathrm{C})$ from phantom lid. Error is greatest at center of phantom, where implant RF heating measurements are generally not made

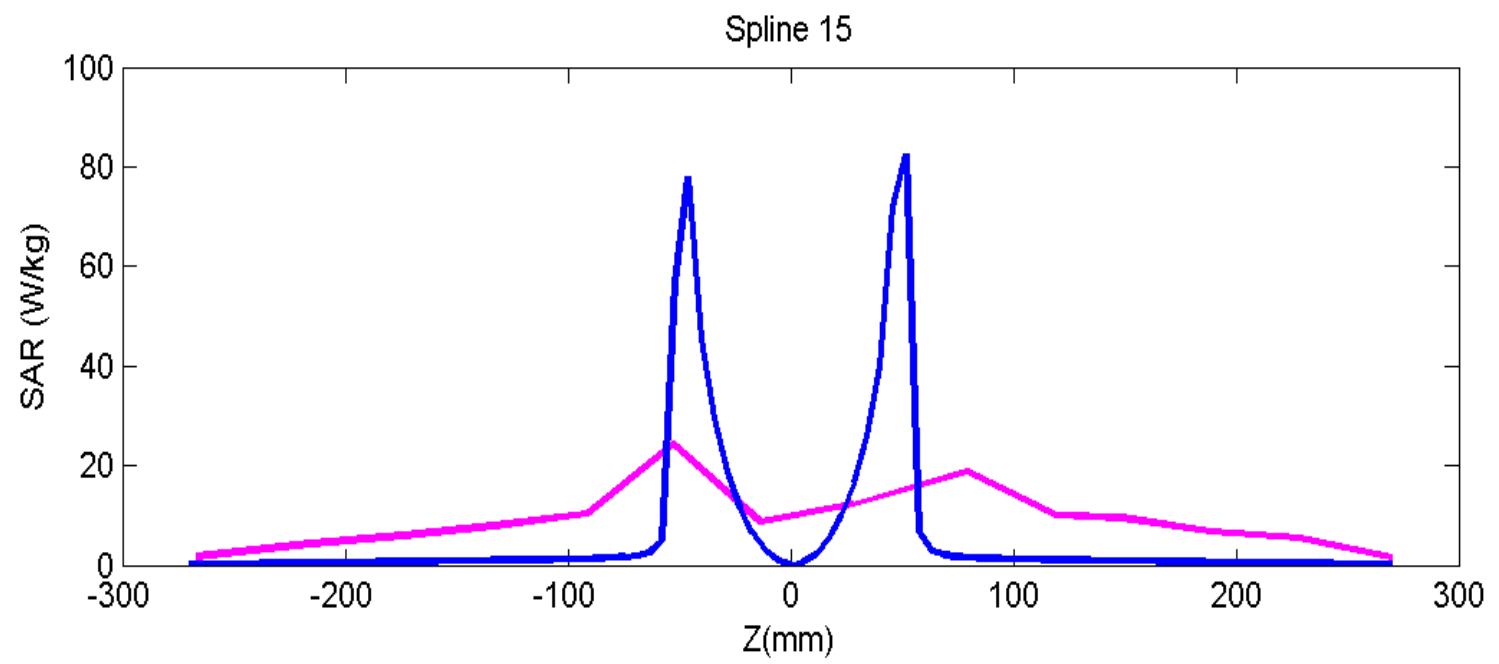

Figure 7.9: Spline 15 (nearest Ti-rod) SAR Measured (pink) and SAR Simulated (blue) $\eta=\mathbf{2 . 9 2 1 4}$, Error $\mathbf{7 1 . 7 \%}$. Due to large gradients, slight mispositioning of probes can easily result in high error. 
Large discrepancy in experimental vs. simulated data at Spline $15($ error $=71.7 \%)$ may be a result of mispositioning of probes. A slight misalignment of a probe along the Z-axis can result in a drastic change in SAR as shown in the simulation plot by a steep fall-off along the Z-axis in the high SAR regions.

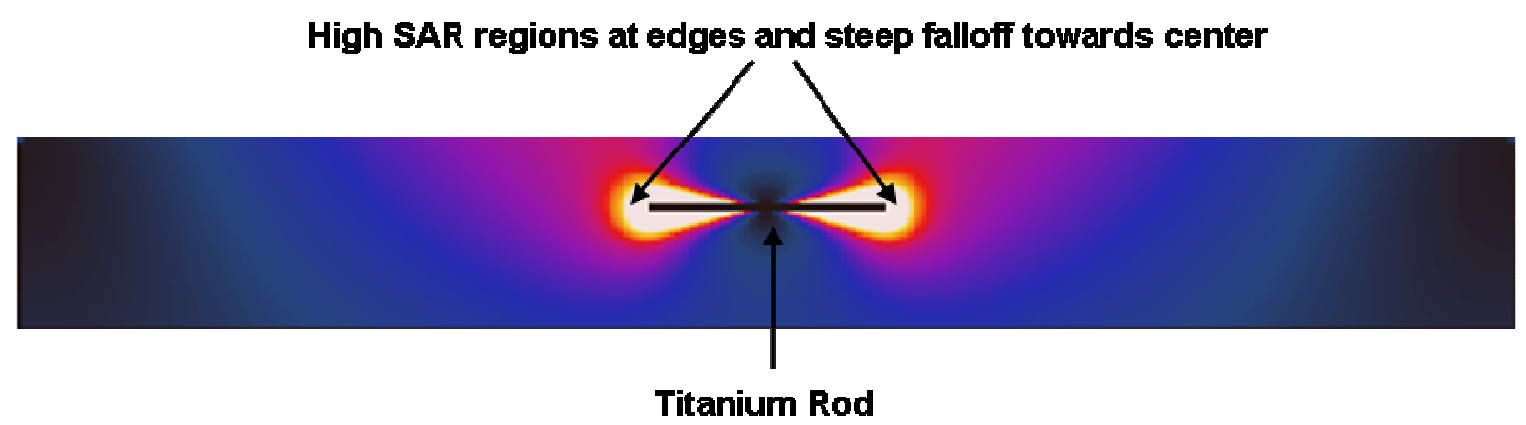

Figure 7.10: Simulated SAR in Ti-Rod region of phantom. Sharp gradients in SAR can cause high error in measurement as a result of slight inaccuracies in probe placement

To explore variability in error when calculating SAR over a specified time range, different RF application time windows were selected when calculating SAR (30-40s, 30130, 30-230, 30-330, etc.), as shown in Figure 5.11. It was determined that measurement error improved initially, but also leveled off pretty quickly with diminishing returns.

Figure 5.11 demonstrates that it may not be necessary to apply RF power much past 1100s. If measurements were to continue to the point where heat transfer dominates, the curve should exhibit an increase in error percentage, as SAR calculations should be made at the initial slope of the curve where it remains linear before heat transport takes place $\mathrm{w}$ within the medium. 




Figure 7.11: To understand error of measured SAR vs. simulated SAR, as a function of different RF application times, SAR was calculated with different time ranges starting at 30s ranging through the 1800s window.

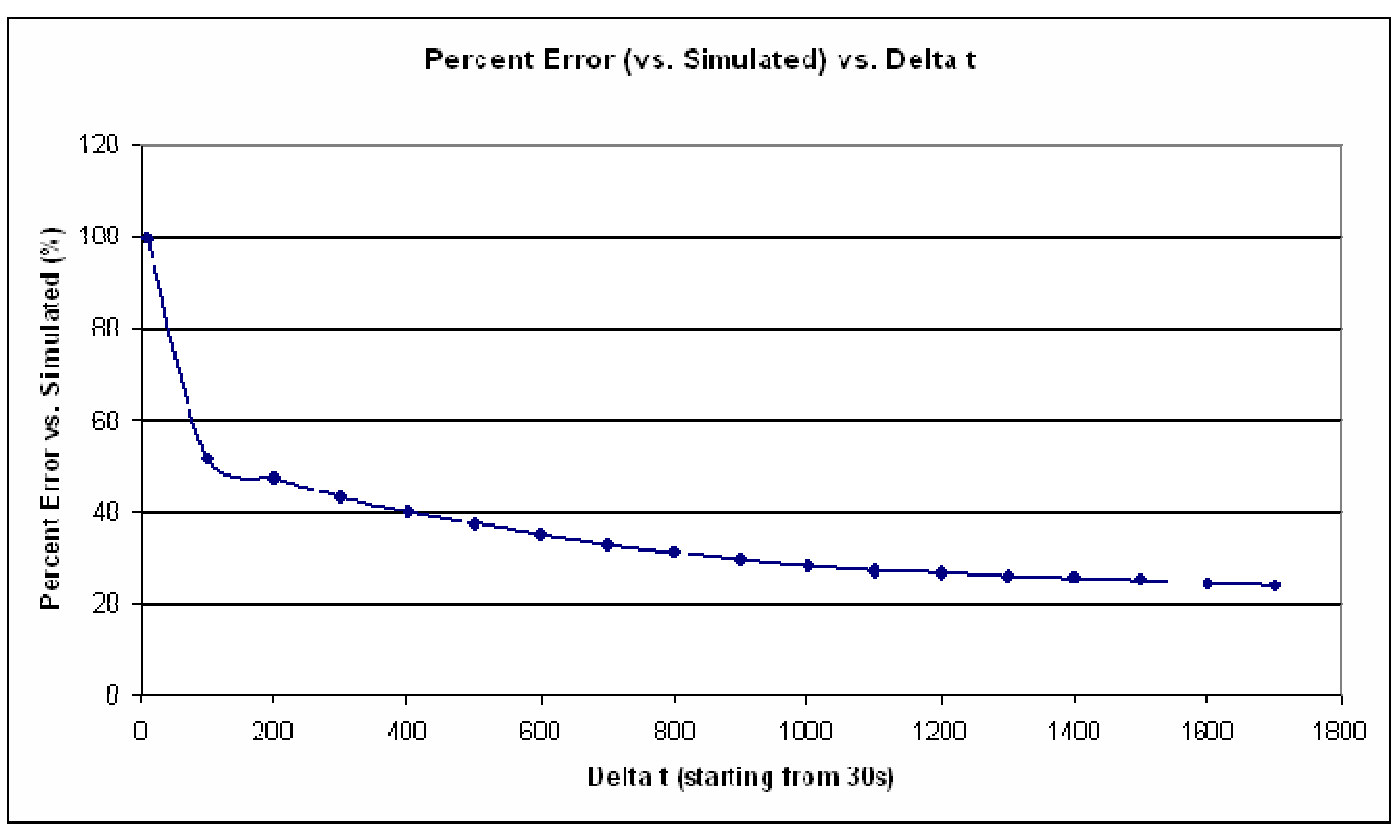

Figure 7.12: Error vs. Delta $t$ (starting from 30s). Initial steep fall off and then diminishing returns indicate that it may not be necessary to apply RF power much past 1100s. 
The lowest error between simulated and measured results was obtained with a time window of 1800 s used to calculate SAR. Over the 1800s, the low SAR regions have enough time to rise significantly in temperature so that it is measurable by the fiber optic probes.

Figure 5.13 illustrates that SAR measurements made in areas of SAR above $\sim .45 \mathrm{~W} / \mathrm{kg}$ using the fiber optic thermometry method, can be measured reasonably accurately for purposes of many RF heating investigations. The error remained below $45 \%$ for measurements made in regions above $.45 \mathrm{~W} / \mathrm{kg}$, whereas the error exponentially grew as SAR got lower. The majority of measurements which were made in the high SAR region are of greatest importance because of the frequency of use of that area in the phantom; hence this study provided accurate results for regions of high SAR, where RF heating tests of implants are generally conducted.

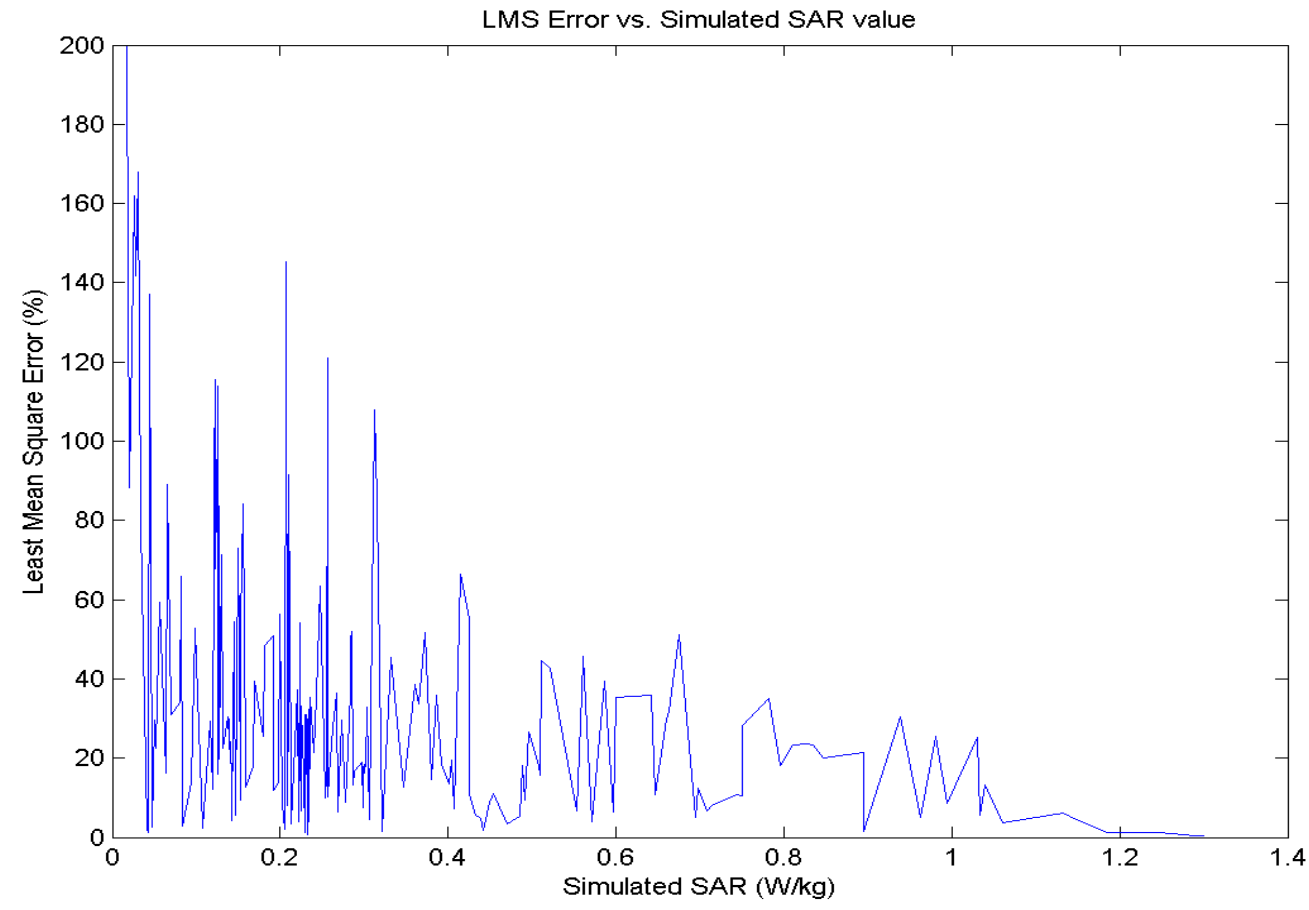

Figure 7.13: LMS Error (Measured SAR) vs. SAR Simulated 


\section{CONCLUSIONS AND FUTURE WORK}

Fiber optic thermometry is a relatively accurate method to determine local background SAR in an ASTM-style phantom, with agreement of 10.7\% with FDTD simulations in the highest SAR region of the phantom (Figure 6.1).
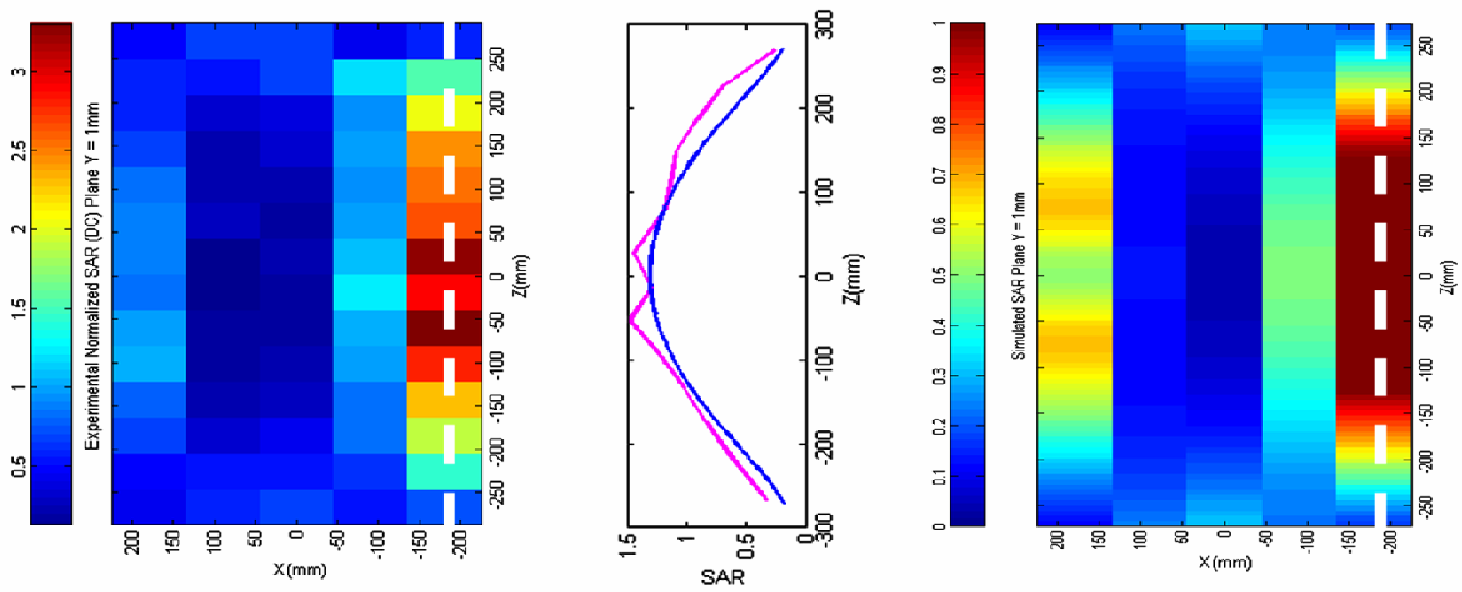

Figure 8.1: Measured (left) and Simulated (right) SAR Distribution (in frequent use region) in ASTM-style phantom

In most experiments, implants are placed in the highest field region in order to expose the device to a worst case scenario. Understanding fields in the area of the phantom where devices are placed is of high importance in an RF exposure experimental setup because:

- The fields to which the device is exposed are the cause of unintended device heating 
- The fields can vary between experimental setups and it is important to understand any differences between setups before experimenting or comparing results

- Need to relate device performance under these fields to performance in fields potentially generated in a clinical setting (in-vivo)

- Understanding field distribution in the phantom without any implants allows us to understand how adding implants may affect the field distribution and how the fields may interact with the implant

Through this work, we find that using the fiber optic thermometry method, the magnitude of these fields in regions where the SAR is above $.45 \mathrm{~W} / \mathrm{kg}$, can be measured relatively accurately (error below 45\%) using long exposure times (1100s or more).

The agreement to simulations proves to be undesirable in regions where the SAR gradient is very high (i.e. ti-rod). In regions where SAR gradient is high, probe placement accuracy becomes the dominating cause of error; hence a very precise method of probe placement would be required. Error in the high gradient region around the ti-rod was $71.7 \%$ which is most likely a result of inaccuracies in probe placement. The overall error obtained when compared to simulations, excluding the ti-rod region was $24.05 \%$. This provides evidence for the assumption that probe placement is the cause of high error in regions of high SAR gradients.

Where the SAR is very low, more towards the center of the phantom the measurement accuracy decreases significantly. The temperature in these areas does not rise above the probe noise floor $\left(.5^{\circ} \mathrm{C}\right)$, without extremely long and unreasonable exposure times. The limit of the measurement equipment is reached and this is the reason for high error percentages when measuring SAR below .45W/kg. Luckily, most medical 
implants are not placed in these regions; hence they are not as relevant for purposes of RF exposure experiments. Practically, this means that highly expensive and more accurate temperature probes and systems are not needed to measure SAR in regions of interest.. A few sources of error which are less significant but still noteworthy include but are not limited to:

- Accuracy of phantom placement inside birdcage (well controlled)

- Change in dielectric properties of phantom with temperature (low temperature rises)

- Variance in power delivered to birdcage (accounted for in normalization of measurements)

With all of this potential for error, obtaining an error of $10.7 \%$ in high field regions using the fiber optic thermometry method is more than achieving the goals of this study. It provides us confidence in the ability to measure background fields in regions of interest, which in turn is directly related to our confidence in metallic implant heating measurements. In an extremely new area of research with relatively few proven options available, the fiber optic thermometry method provides high accuracy.

Drawbacks of the method are that it is tedious and requires substantial time and effort to get a high resolution picture of SAR distribution over a large volume. As pointed out earlier, the highest sources of error consist of probe placement accuracy and equipment limits (fiber optic temperature probe measurement resolution). With these sources of error in mind, this method is capable of providing satisfactory results for an experimental environment. 
Future studies may involve using field probes to measure SAR via E-field in the medium and comparing the accuracy of the method to the fiber optic thermometry method. The phantom medium used herein $\left(\sigma=.49 \mathrm{~S} / \mathrm{m}\right.$ and $\left.\varepsilon_{\mathrm{r}}=78\right)$ may change as a result of attempts to understand implants located in different regions of the body (i.e. fat or muscle tissue).It would be beneficial to industry to understand the capability of the fiber optic thermometry method across various phantom media.

A study to understand the sensitivity of the fields to the position of the phantom in the birdcage would provide insight into how important it is to accurately position the phantom in the birdcage. Also, the depth to which the phantom needs to be filled to produce the intended fields would provide valuable information to those conducting studies in phantoms.

Finally, though this method proved to be quite accurate, it exhibited poorer performance in low SAR regions of the phantom below $.45 \mathrm{~W} / \mathrm{kg}$. A solution to this may be to use either a SAR probe which measures SAR via E-fields, or using a fiber optic temperature measurement system with higher resolution. 


\section{REFERENCES}

[1] Park, S.M., et al. "MRI safety: RF-induced heating near straight wires", Magnetics, IEEE Transactions, 41(10), Oct. 2005

[2] "Standard Test Method for Measurement of Radio Frequency Induced Heating on or Near Passive Implants During Magnetic Resonance Imaging”, ASTM International Standard f2182-09, Nov. 2009

[3] Boston Scientific, Inc., Available: http://www.controlyourpain.com/. [Accessed February 25, 2011]

[4] Medtronic, Inc., Available: http://www.medtronic.com/. [Accessed February 25, 2011]

[5] St. Jude Medical, Inc., Available: http://sjm.com/. [Accessed February 25, 2011]

[6] Medtronic, "MRI Safety for Implantable Cardiac Devices”, September 24, 2010. [Online]. Available: http://www.medtronic.com/for-healthcare-

professionals/products-therapies/cardiac-rhythm/cardiac-monitors-insert/mrisafety-for-implantable-cardiac-devices/index.htm. [Accessed December 12, 2010]. 
[7] Henderson, J., et al., "Permanent Neurological Deficit Related to Magnetic Resonance Imaging in a Patient with Implanted Deep Brain Stimulation Electrodes for Parkinson's Disease: Case Report", Neurosurgery Vol. 57 No. 5 , November 2005

[8] Mattei, E., et al., "MRI induced heating of pacemaker leads: effects of temperature probe positioning and pacemaker placement on lead tip heating and local SAR", Proceeding of the $28^{\text {th }}$ IEEE EMBS Conference, p.1889, August 2006

[9] Baker, K., et al., "Evaluation of Specific Absorption Rate as a Dosimeter of MRIRelated Implant Heating", Journal of Magnetic Resonance Imaging Vol. 20 315320,2004

[10] Nordbeck, P., et al., "Spatial Distribution of RF-Induced E-Fields and Implant Heating in MRI", Magnetic Resonance in Medicine 60, p. 312-319, 2008

[11] IT'IS Foundation, Zeughausstrasse 43, CH-8004 Zurich, Switzerland. Available: http://www.itis.ethz.ch/services/human-and-animal-models/human-models/

[12] Gururaj, K., Weerakoon, P., et al., "Detecting and Preventing MRI Gradient Field Currents", October 2010

[13] Jin, Jian-Ming, Electromagnetic Analysis and Design in Magnetic Resonance Imaging. Florida: CRC Press, 1999.

[14] Wikipedia contributors, "Finite-difference time-domain method", Wikipedia, The Free Encyclopedia, October 26, 2010. [Online]. Available: http://en.wikipedia.org/wiki/Finite-difference_time-domain_method. [Accessed December 12, 2010] 
[15] Schmid \& Partner Engineering AG, "SEMCAD X Reference Manual”, April 2010

[16] Schmid \& Partner Engineering AG, SEMCAD X (Version 14.2) [Software]. 2010

[17] Kawamura, T., et al., "Specific Absorption Rate Measurement of Birdcage Coil for 3.0-T Magnetic Resonance Imaging System Employing Thermographic Method", IEEE Transactions on Microwave Theory and Techniques Vol. 57, No. 10, p. 2508 , October 2009

[18] Katscher, U., et al., "Determination of Electrical Conductivity and Local SAR Via B1 Mapping”, IEEE Transactions on Medical Imaging Vol. 28 No. 9, September 2009

[19] Jallal, J.A., et al., "Standard Implant for Determination of Local SAR in Testing of RF-induced Heating of an Implant”, Proceedings of ISMRM 17, p.4799, May 2009 


\section{APPENDIX - RAW TEMPERATURE DATA}

The following raw data is presented as temperature rise over time for individual points and is grouped with splines. Each spline represents a series of 14 measurement points parallel to the long edge of the phantom. For each plot, $\mathrm{P} 1_{\mathrm{M} 1}$ is monitoring the temperature rise at one end of the ti-rod, and $\mathrm{P} 3_{\mathrm{M} 4}$ is monitoring the ambient temperature of the room. Both of these measurements can be neglected as they are not used in this study. A measurement grid is displayed below in Figure 8.1 for reference. Please refer to the grid to determine spline and measurement point locations.



Figure 10.1: Temperature Measurement Grid (Splines 1-15) 

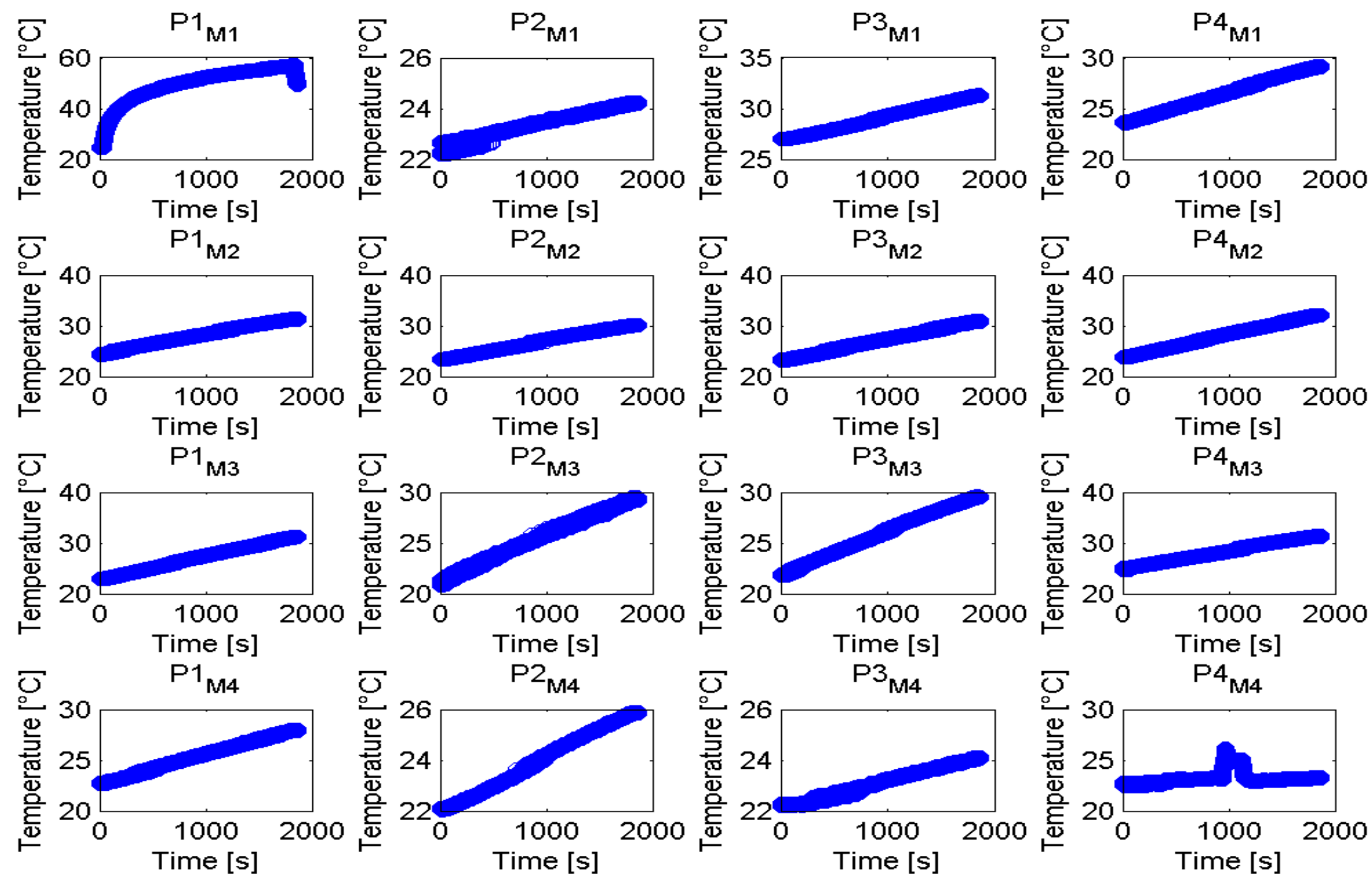

Figure 10.2: Spline 1 Raw Temperature Data P2M1 - P3M4 (14 Probes per Spline) 



Figure 10.3: Spline 2 Raw Temperature Data P2M1 - P3M4 (14 Probes per Spline) 

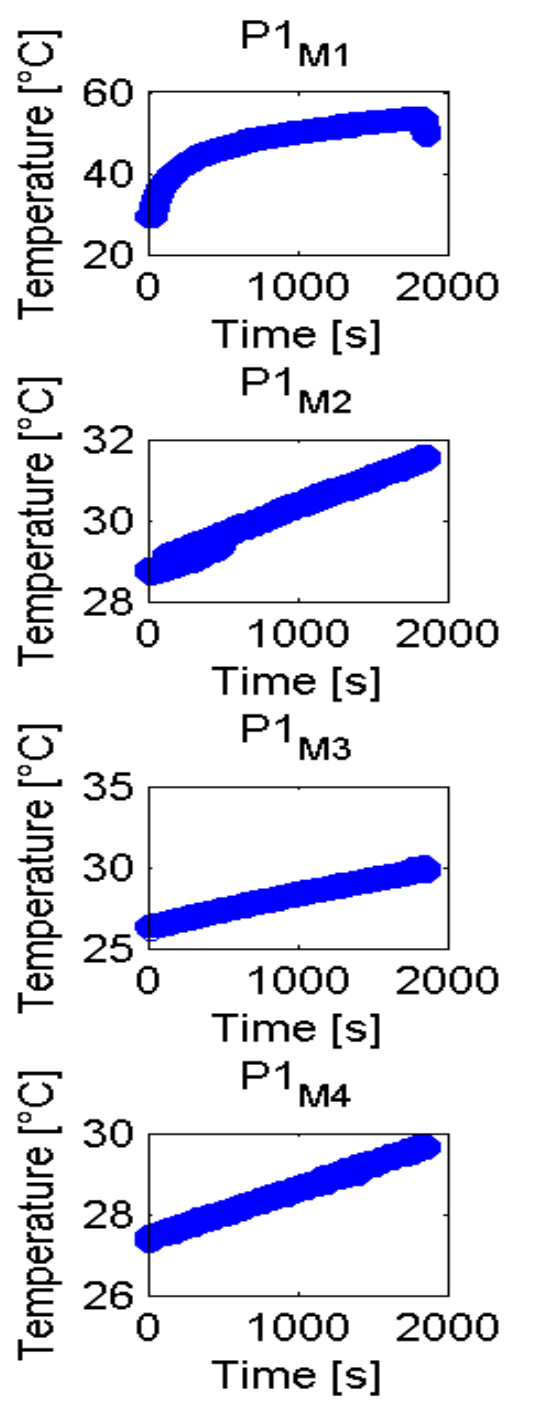
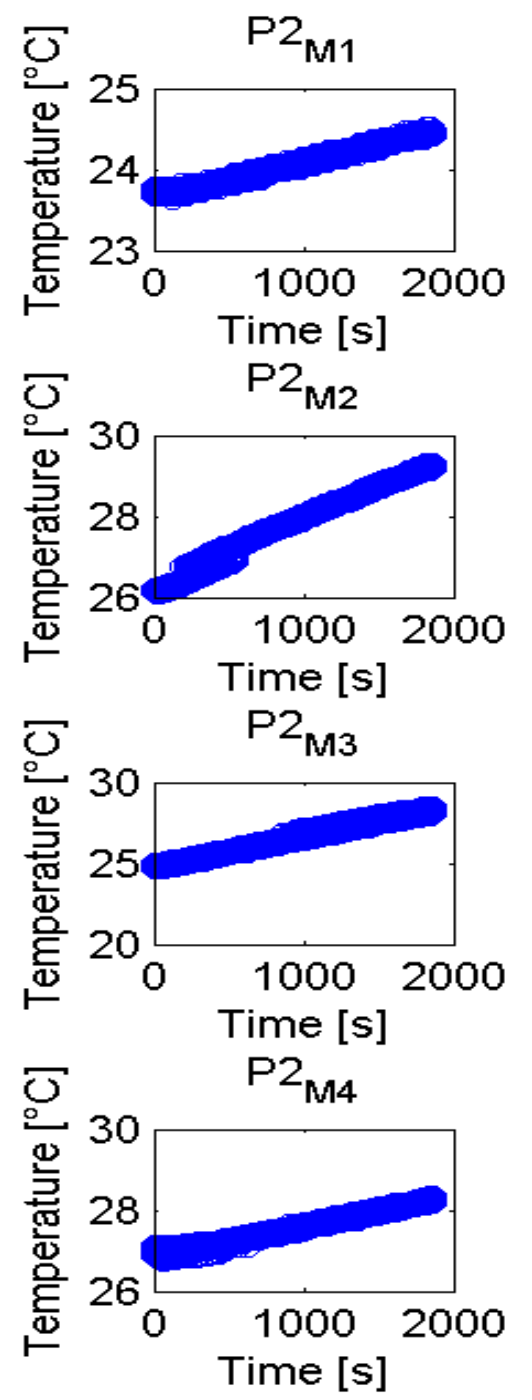
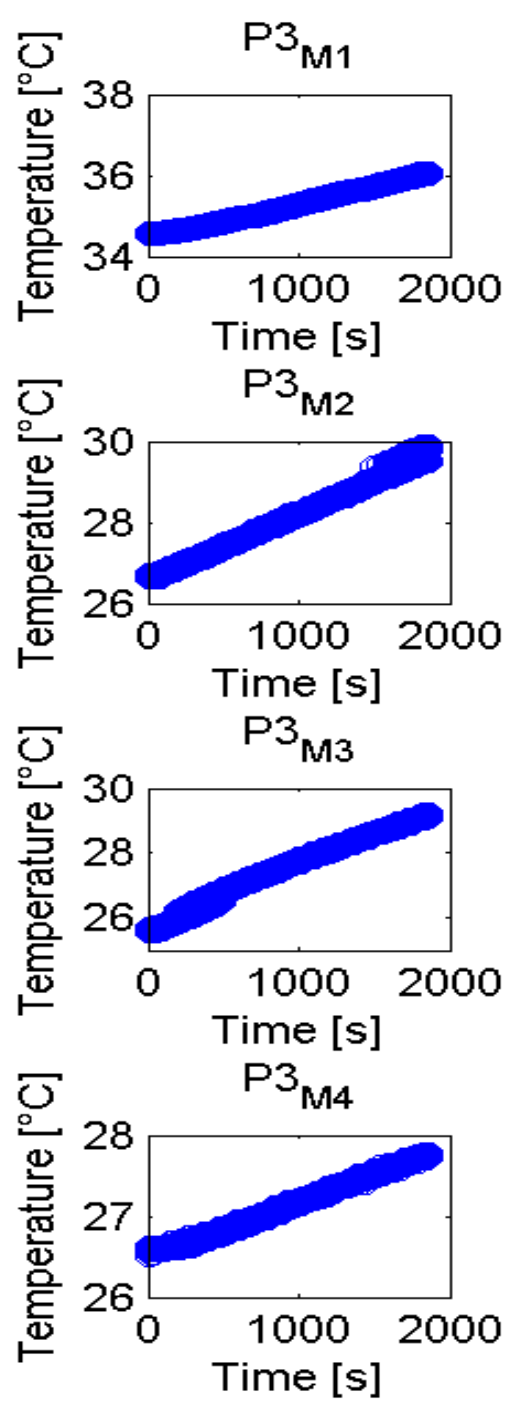
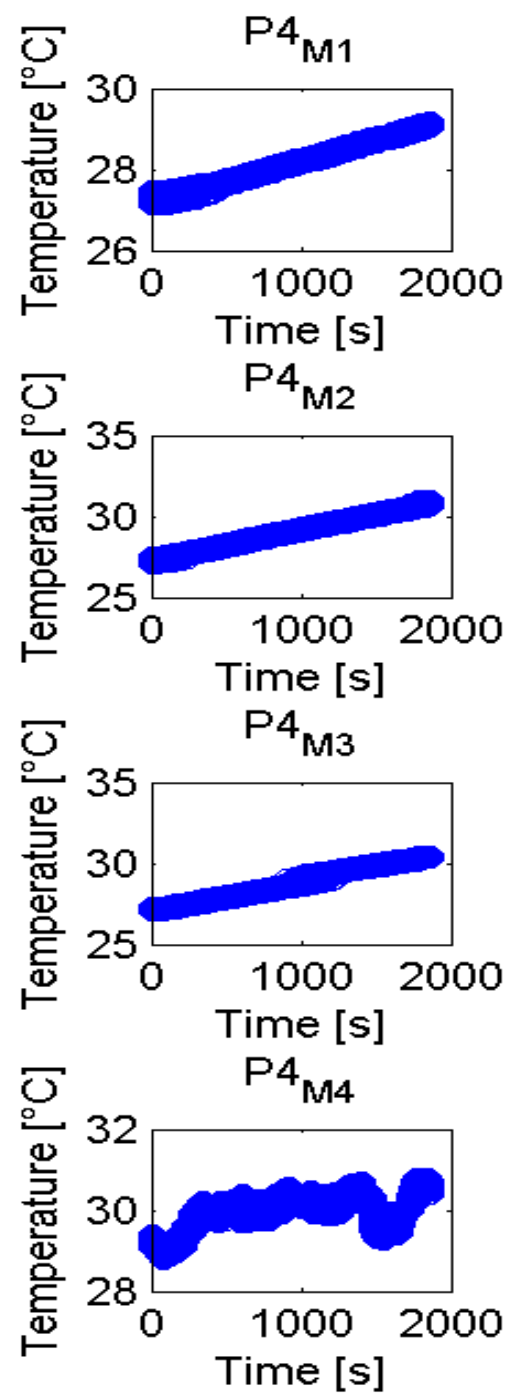

Figure 10.4: Spline 3 Raw Temperature Data P2M1 - P3M4 (14 Probes per Spline) 

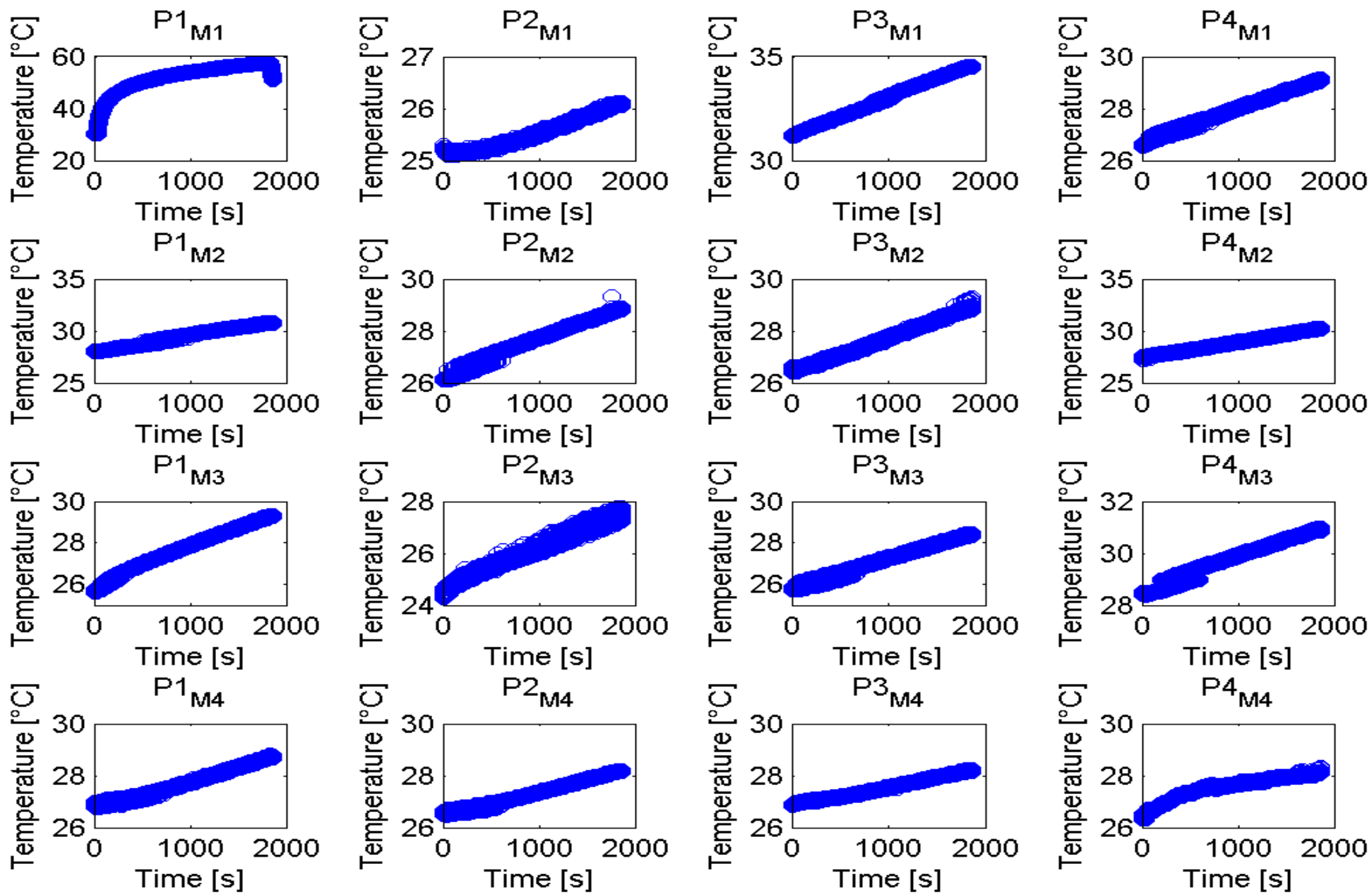

Figure 10.5: Spline 4 Raw Temperature Data P2M1 - P3M4 (14 Probes per Spline) 

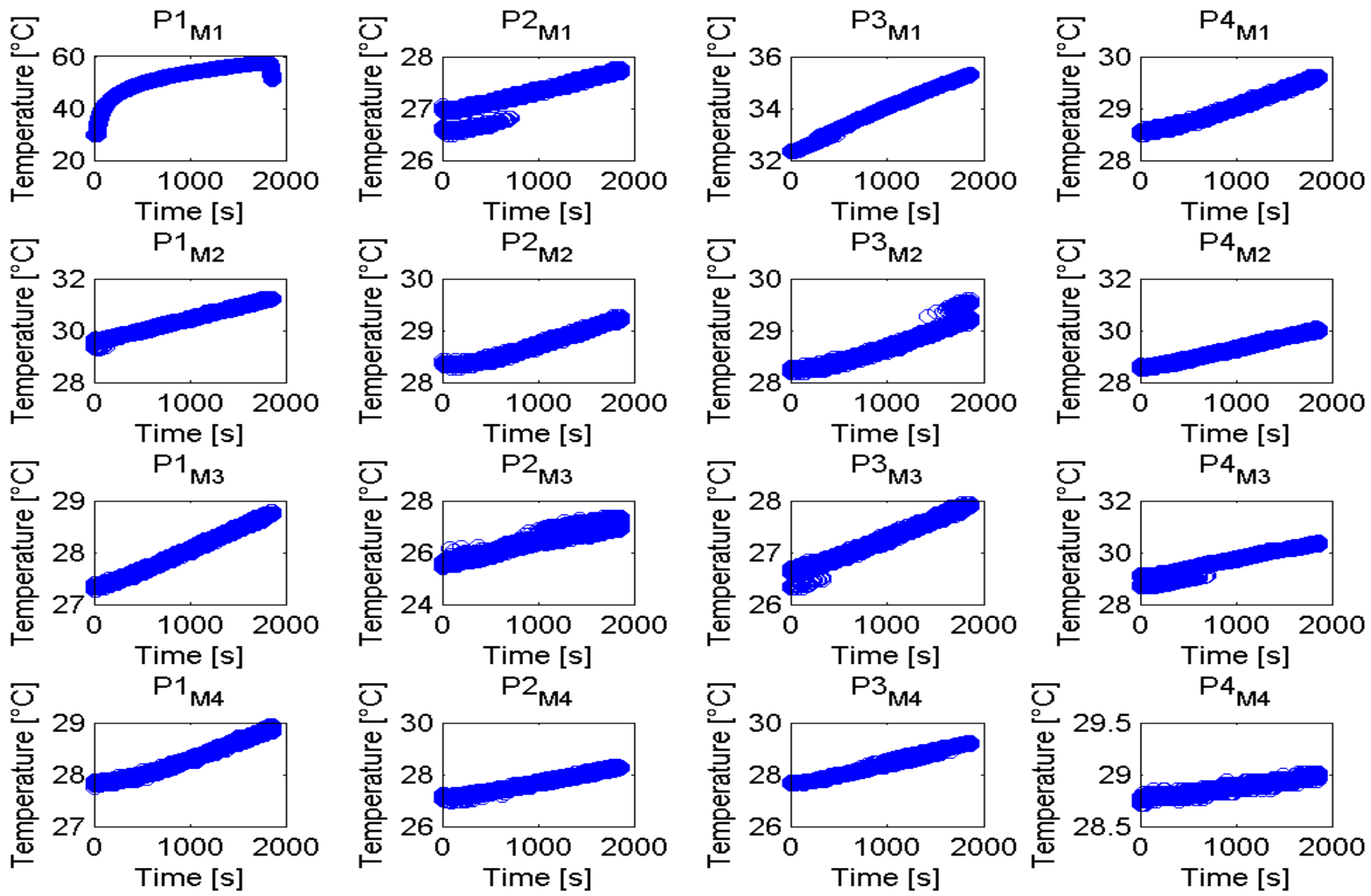

Figure 10.6: Spline 5 Raw Temperature Data P2M1 - P3M4 (14 Probes per Spline) 

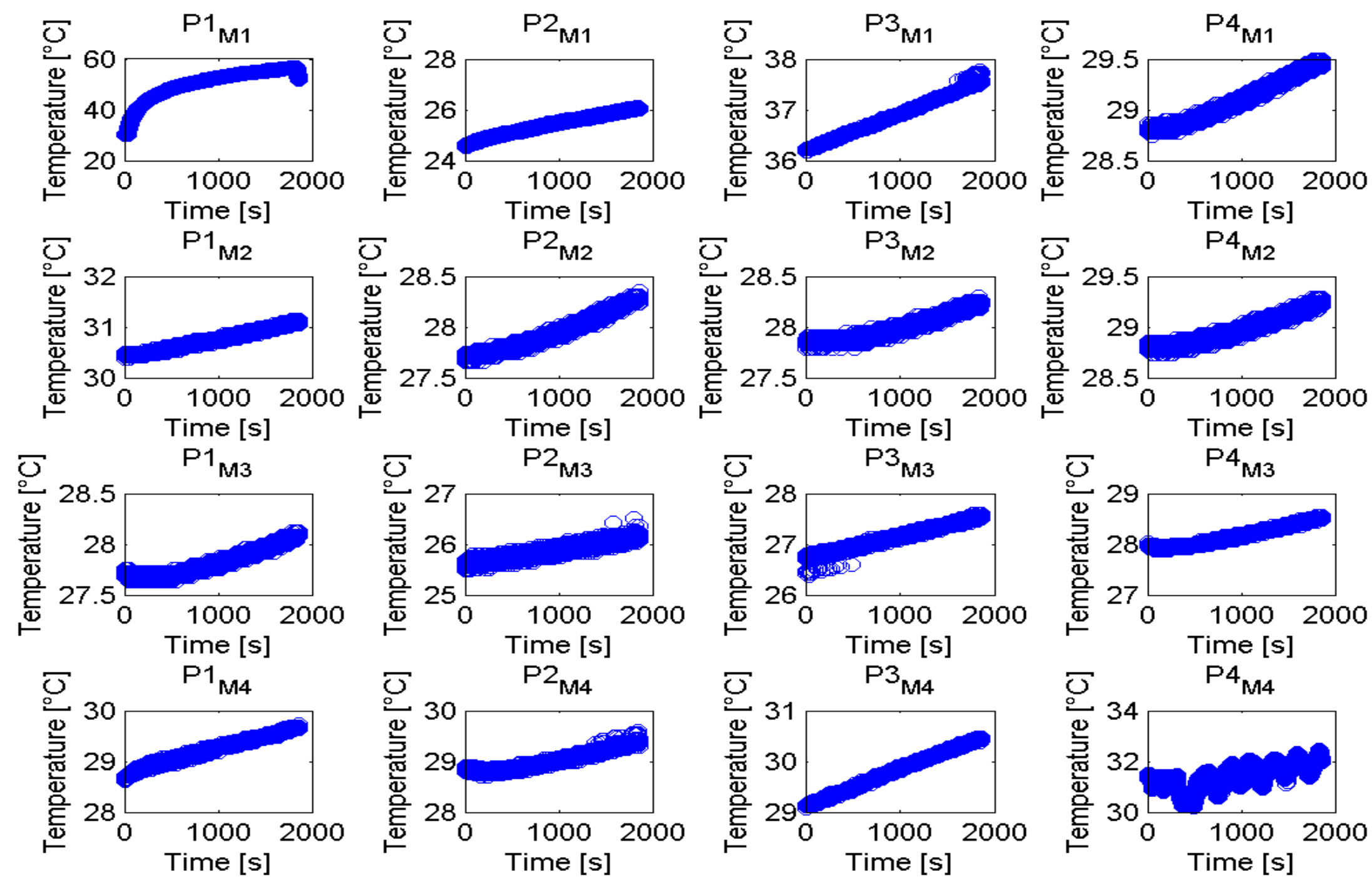

Figure 10.7: Spline 6 Raw Temperature Data P2M1 - P3M4 (14 Probes per Spline) 

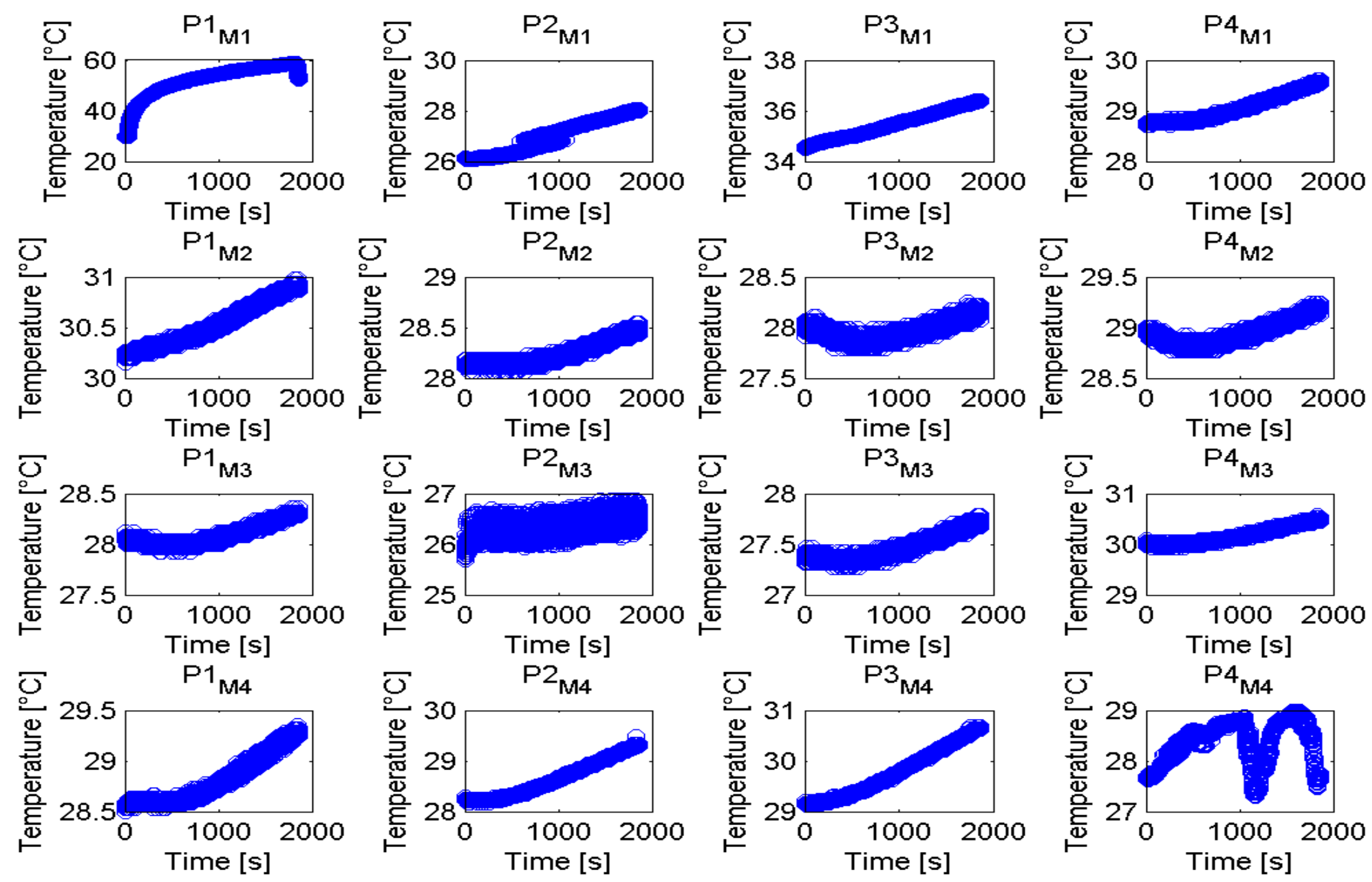

Figure 10.8: Spline 7 Raw Temperature Data P2M1 - P3M4 (14 Probes per Spline) 

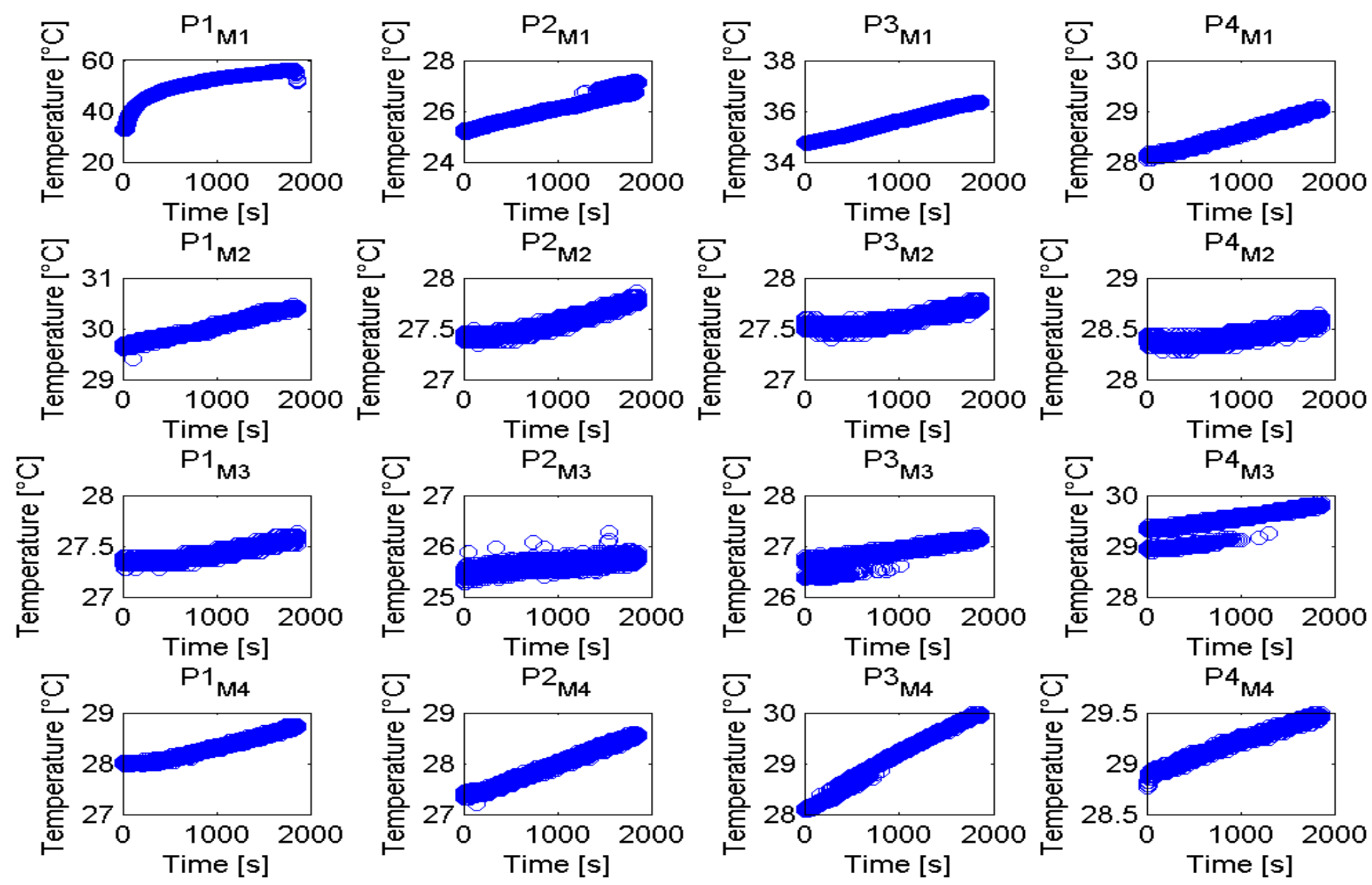

Figure 10.9: Spline 8 Raw Temperature Data P2M1 - P3M4 (14 Probes per Spline) 



Figure 10.10: Spline 9 Raw Temperature Data P2M1 - P3M4 (14 Probes per Spline) 


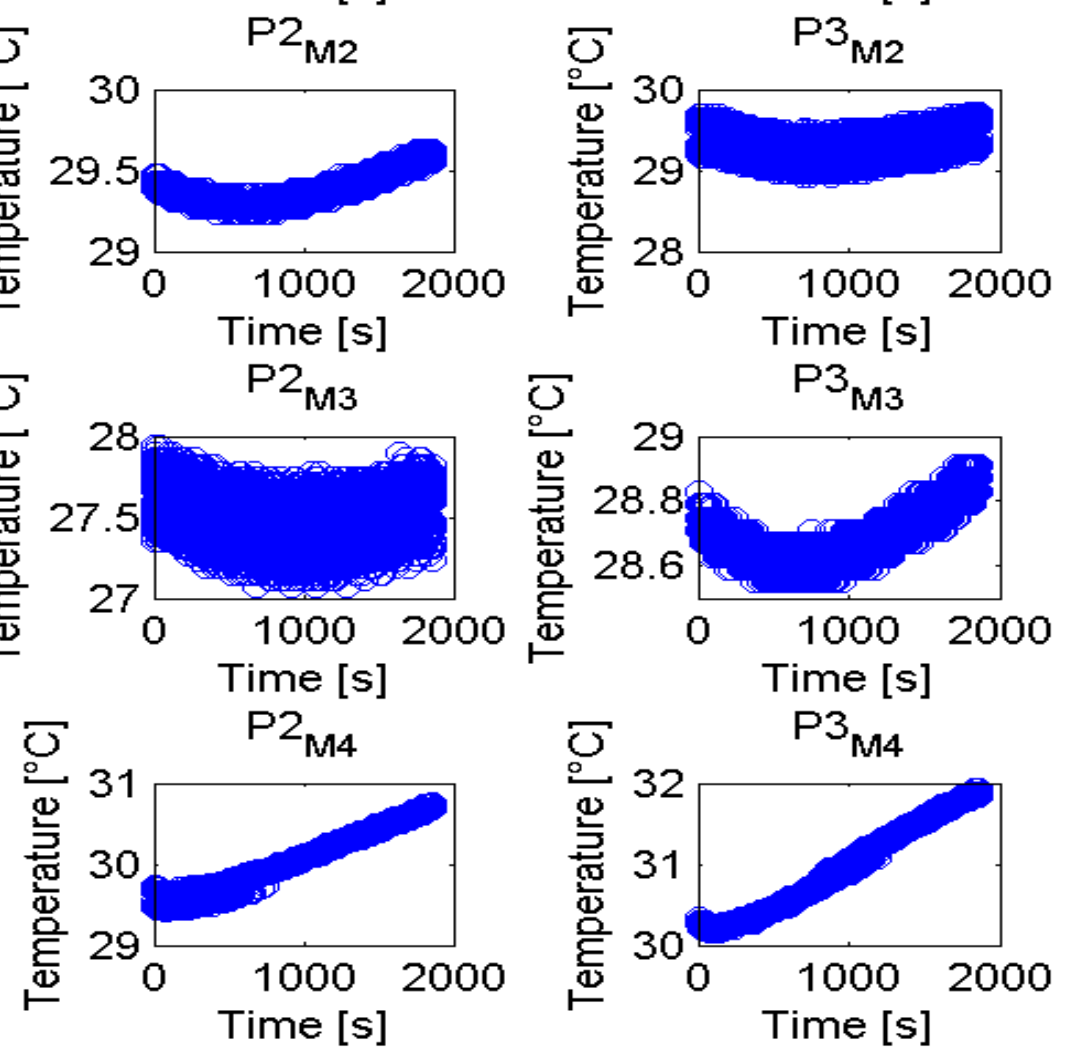
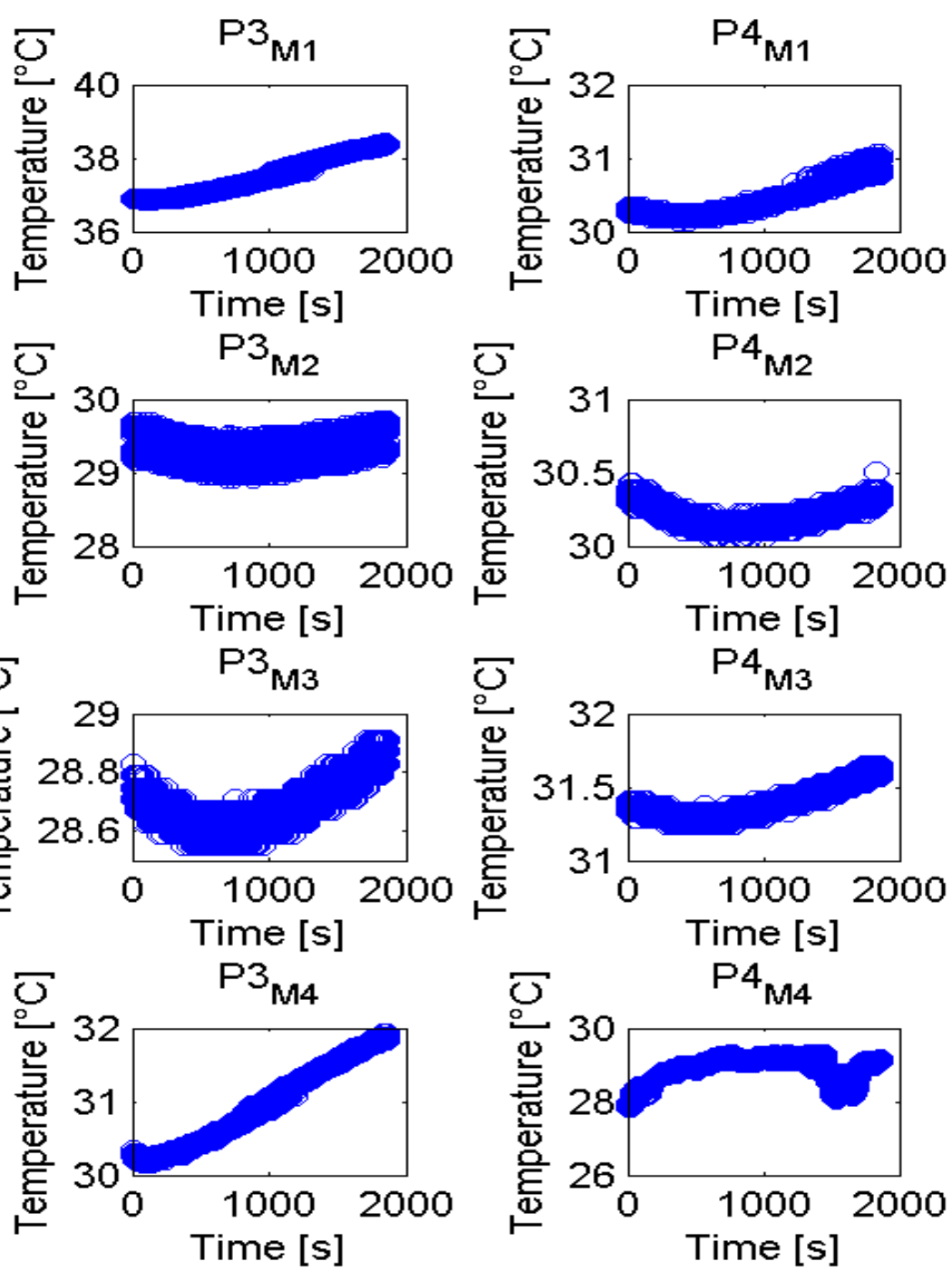

Figure 10.11: Spline 10 Raw Temperature Data P2M1 - P3M4 (14 Probes per Spline) 

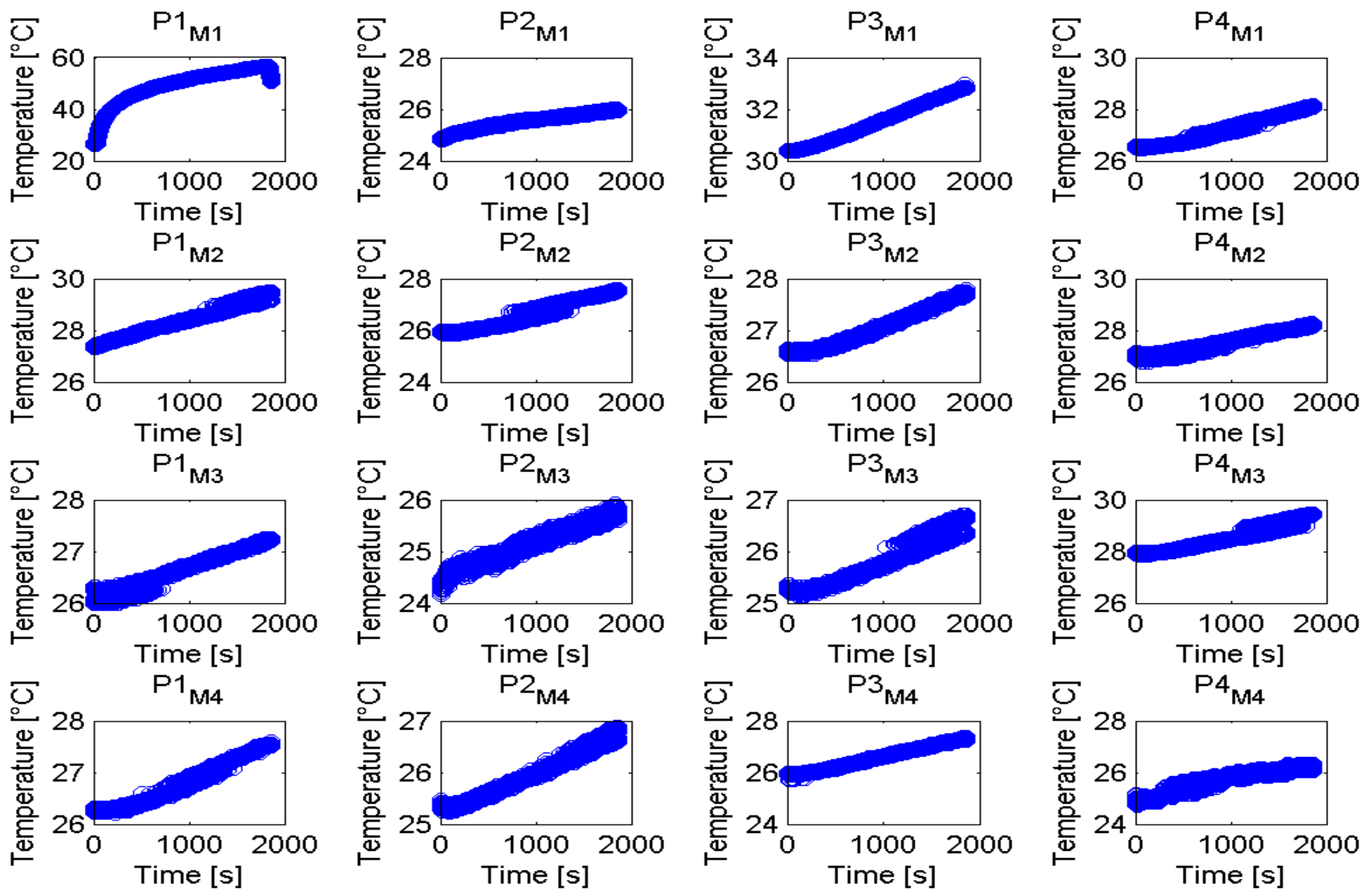

Figure 10.12: Spline 11 Raw Temperature Data P2M1 - P3M4 (14 Probes per Spline) 


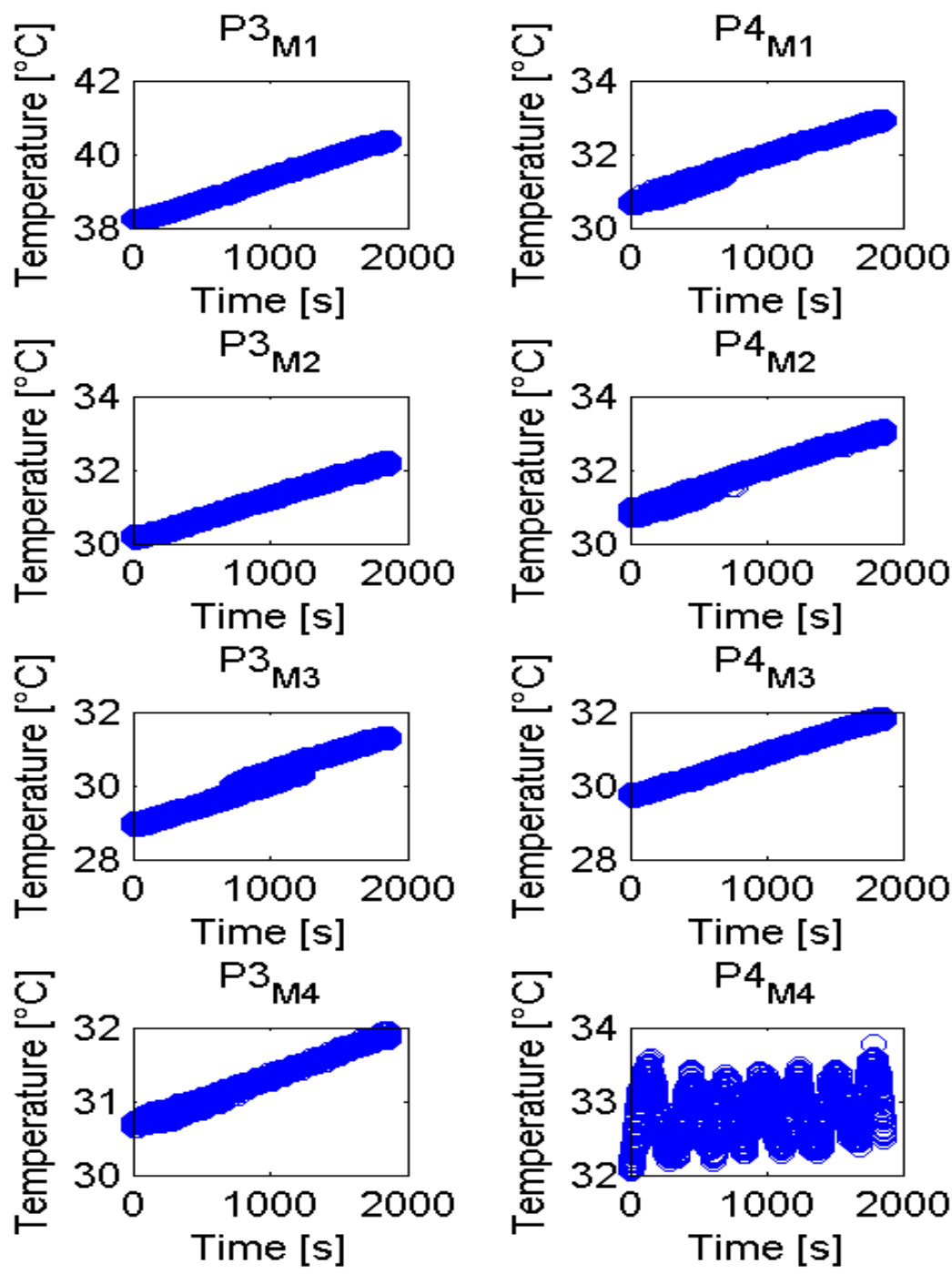

Figure 10.13: Spline 12 Raw Temperature Data P2M1 - P3M4 (14 Probes per Spline) 



Figure 10.14: Spline 13 Raw Temperature Data P2M1 - P3M4 (14 Probes per Spline) 

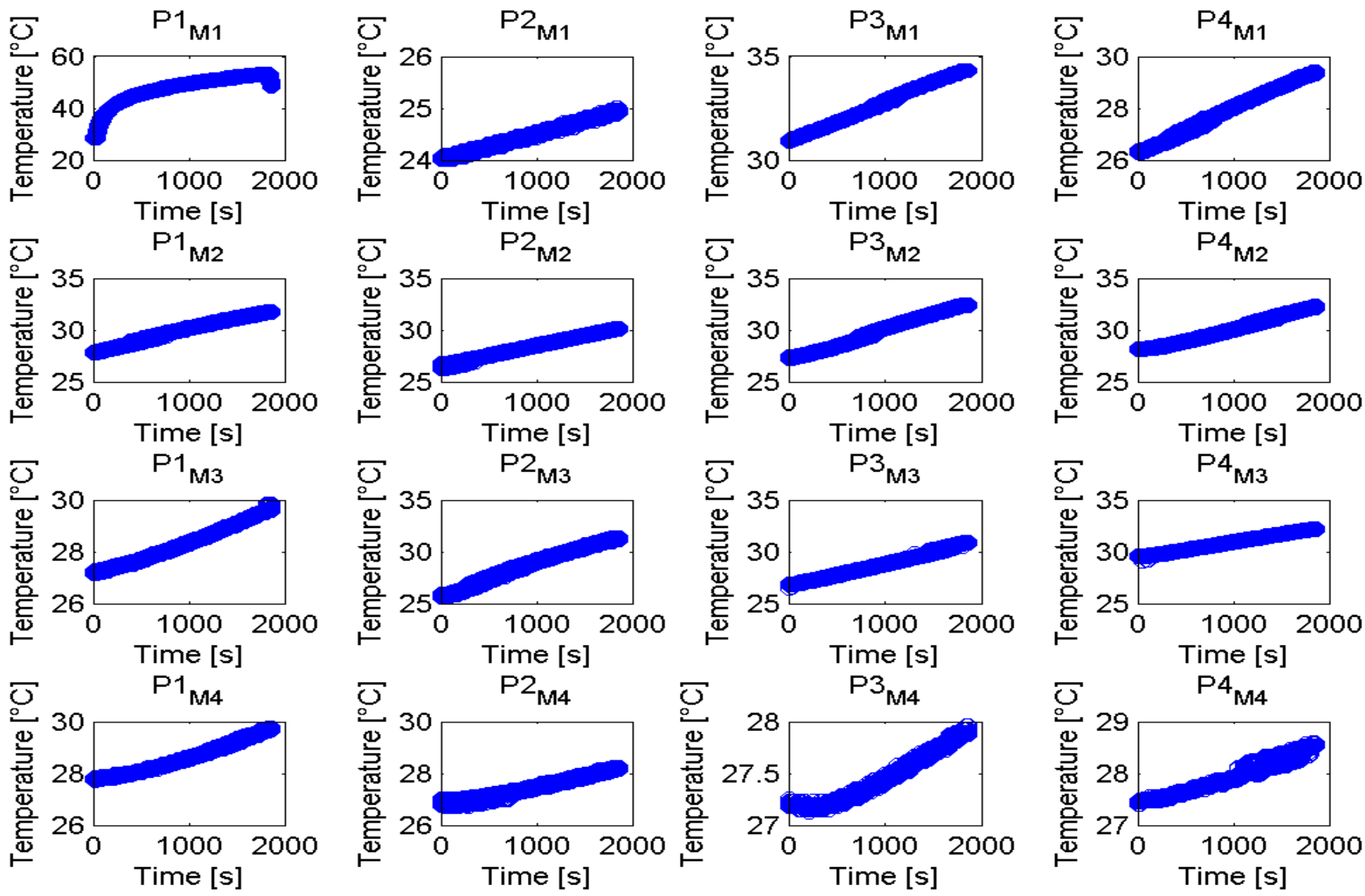

Figure 10.15: Spline 14 Raw Temperature Data P2M1 - P3M4 (14 Probes per Spline) 



Figure 10.16: Spline 15 Raw Temperature Data P2M1 - P3M4 (14 Probes per Spline) 